\title{
Surveillance for Cancer Incidence and Mortality - United States, 2012
}

\author{
Simple D. Singh, $\mathrm{MD}^{1}$ \\ S. Jane Henley, $\mathrm{MSPH}^{1}$ \\ A. Blythe Ryerson, $\mathrm{PhD}^{1}$ \\ ${ }^{1}$ Division of Cancer Prevention and Control, National Center for Chronic Disease Prevention and Health Promotion, CDC
}

\section{Preface}

This report provides, in tabular and graphic form, official federal statistics on the occurrence of cancer for 2012 and trends for 1999-2012 as reported by CDC and the National Cancer Institute (NCI) (1). Cancer incidence data are from population-based cancer registries that participate in CDC's National Program of Cancer Registries (NPCR) and NCI's Surveillance, Epidemiology, and End Results (SEER) program reported as of November 2014. Cancer mortality data are from death certificate information reported to state vital statistics offices through 2012 and compiled into a national file for the entire United States by CDC's National Center for Health Statistics' (NCHS) National Vital Statistics System (NVSS). This report is a part of the Summary of Notifiable Noninfectious Conditions and Disease Outbreaks - United States, which encompasses various surveillance years but is being published in 2016 (2). The Summary of Notifiable Noninfectious Conditions and Disease Outbreaks appears in the same volume of MMWR as the annual Summary of Notifiable Infectious Diseases (3).

This report presents information on new cancer cases and deaths for 2012. The number and rate of cancer cases and deaths are stratified by the primary cancer sites as reported for 2012; information is provided by demographic characteristic (e.g., sex, age, race, and ethnicity) and primary cancer site (68 selected sites among men and 72 selected sites among women). Age-adjusted cancer incidence and death rates are shown by primary site and year for the period 1999-2012. Ageadjusted cancer incidence and death rates for the most common sites are shown by race, sex, and ethnicity for 2012, the most recent year for which incidence data are available. Maps of the United States display age-adjusted cancer incidence and death rates, presented by quartiles, for 2012. Time trends in ageadjusted cancer incidence and death rates during 1999-2012 are shown by race, sex, and ethnicity for all sites combined, colorectal, lung and bronchus, prostate, and female breast.

Corresponding author: Simple Singh, Division of Cancer Prevention and Control, National Center for Chronic Disease Prevention and Health Promotion, CDC. Telephone: 770-488-4292; E-mail: sdsingh@cdc.gov.

\section{Background}

Cancer comprises a diverse mix of diseases occurring in every part of the body and is a leading cause of death in the United States (4). More than half of cancer cases could be prevented (5). Surveillance of cancer incidence and mortality can help public health officials target areas for control efforts $(G)$ and track progress toward meeting the national health objectives set forth in Healthy People 2020 (7). Cancer is a reportable disease in every state and thus all hospitals, physicians' offices, pathology laboratories, and other medical facilities are required to submit data on all reportable cancer diagnoses to a central cancer registry at the state or territorial level. A cancer registry is a database that contains individual records of all reportable cancer cases in a defined population and includes patient demographics, tumor characteristics (e.g., cancer site and pathology), and information about the notifying health provider or facility. In 1992, Congress established NPCR by enacting the Cancer Registries Amendment Act (Public Law 102-515) (8). Administered by CDC, NPCR collects data on the occurrence of cancer and the type, extent, and location of the cancer. Before NPCR was established, 10 states had no registry, and most states with registries lacked the resources and state legislation needed to gather complete data (9). Presently, NPCR supports central cancer registries in 45 states, the District of Columbia (DC), Puerto Rico, and the U.S. Pacific Island Jurisdictions. NPCR data represent $96 \%$ of the overall U.S. population. Together, NPCR and NCI's SEER Program collect data for the entire U.S. population. Cancer control planners and others can identify variations in cancer rates by population subgroups and monitor trends over time to guide the planning and evaluation of cancer prevention and control programs and allocation of health resources.

\section{Data Sources}

Data about cancer incidence and mortality in the Summary of Notifiable Noninfectious Conditions and Disease Outbreaks come from the official federal statistics on cancer, the U.S. Cancer Statistics (USCS) dataset (1). The USCS dataset includes cancer incidence data from NPCR registries in 45 states and 
DC (cancer incidence data from Puerto Rico and the U.S. Pacific Island Jurisdictions were not available for this analysis) and from SEER program registries in the remaining five states (Connecticut, Hawaii, Iowa, New Mexico, and Utah) and cancer mortality data from NVSS.

\section{Incidence Data}

The primary source of data on cancer incidence is medical records. Staff at health care facilities abstract data from patients' medical records, enter it into the facility's own cancer registry if it has one, and then send the data to the regional or state registry. Both NPCR and SEER registries collect data using uniform data items and codes as documented by the North American Association of Central Cancer Registries (NAACCR). This uniformity ensures that data items collected by the two federal programs are comparable $(10,11)$. Information on primary site and histology is coded according to the International Classification of Diseases for Oncology, Third Edition (ICD-O-3) and categorized according to the revised SEER recodes dated January 27, 2003, which define standard groupings of primary cancer sites (http://seer.cancer. gov/siterecode) (12). Beginning with 2010 diagnoses, cases were first classified by anatomic site using ICD-O-3; cases with hematopoetic histologies were further classified by using the 2008 WHO Classification of Tumours of Haematopoietic and Lymphoid Tissues (13). Data from the NPCR registries provided in this report were reported to CDC as of November 30, 2014. Data from SEER registries were reported to NCI as of November 1, 2014.

NPCR and SEER cancer registries consider as reportable all incident cases with a behavior code of 2 (in situ, noninvasive) or 3 (malignant, primary site only) in ICD-O-3. Exceptions include in situ cancer of the cervix and all basal and squamous cell carcinomas of the skin, except for those on the skin of the genital organs (12). Beginning with 2001 diagnoses, several cancers that are coded as malignant in ICD-O-3 were not coded as malignant in ICD-O-2 (10). Additional information is provided in the USCS technical notes (http://www.cdc.gov/ cancer/npcr/uscs/pdf/uscs-2012-technical-notes.pdf\#namedd est=IncidenceDataSources).

\section{Mortality Data}

Cancer mortality statistics are based on information from all death certificates filed in the 50 states and DC and processed by NVSS at NCHS (14). The cancer mortality data were compiled in accordance with World Health Organization regulations, which specify that member nations classify and code causes of death in accordance with the current revision of the International Classification of Diseases (ICD) (15). For consistency with the data on cancer incidence, the cancer sites in mortality data were grouped according to the revised SEER recodes dated January 27, 2003 (available at http://seer.cancer. gov/codrecode). Data for a specific calendar year are based on records of deaths that occurred during that calendar year and received by a particular date; for example, mortality data for 2012 are based on records of deaths that occurred during 2012 and were received as of June 30, 2014. Additional information is provided in the USCS technical notes (http://www.cdc.gov/ cancer/npcr/uscs/pdf/uscs-2012-technical-notes.pdf\#namedd est=MortalityDataSources).

\section{Population Estimates}

Population denominators are race-specific, ethnicity-specific, and sex-specific county population estimates from the U.S. Census, modified by SEER and aggregated to the state and national level. Additional details about population data are available at http://seer.cancer.gov/popdata/index.html.

\section{Interpreting Data \\ Incidence Data}

Each year, state cancer registries submit cancer cases for a new diagnosis year and an updated version of the previous years' cancer cases to CDC or NCI. Therefore, each year, when USCS data are published, updates to the previous year's data are published, using the most recent data submission and the most recent population data. Users of cancer incidence data published by federal agencies should be mindful of the data submission dates for all data used in their analyses.

\section{Mortality Data}

Cancer mortality statistics in USCS are influenced by the accuracy of information on the death certificate. Unlike incidence data, mortality data for a calendar year are considered complete when submitted and so are not updated after the aggregate data file is released. Mortality data for the entire United States refer only to deaths that occurred within the United States; data for geographic areas are provided by the decedent's place of residence.

\section{Race and Ethnicity Data}

For cancer incidence, race and ethnicity data are abstracted from medical records and grouped into categories (11). When cancer mortality is reported, race and ethnic origin are recorded separately on the death certificate by the funeral director as 
provided by an informant or, in the absence of an informant, on the basis of observation (16).

Differences in rates among racial and ethnic populations should be interpreted with caution. A study using SEER incidence data suggests that the quality of data on race in cancer registries is considered excellent for whites, blacks, and Asians/Pacific Islanders, good for Hispanics, and poor for American Indians/Alaska Natives (17). Previous studies involving cancer mortality data demonstrate that death rates for whites and blacks generally are estimated accurately whereas death rates for Asians/Pacific Islanders, American Indians/ Alaska Natives, and Hispanics are underestimated (18). For this reason, incidence and mortality data provided in this report might be underestimated for these groups, possibly because of misclassification of race or Hispanic ethnicity.

Three NPCR registries (Delaware, Kentucky, and South Carolina) opted not to present state-specific Asian/Pacific Islander counts and rates. Five NPCR registries (Delaware, Kentucky, Massachusetts, Pennsylvania, and South Carolina) opted not to present state-specific Hispanic (classified by the NAACCR Hispanic Identification [NHIA] Algorithm) counts and rates (19). Cancer registries regularly link their database to the Indian Health Service patient registration dataset to reduce misclassification of race for American Indian/Alaska Native cases. Seven NPCR registries (Delaware, Illinois, Kansas, Kentucky, New Jersey, New York, and South Carolina) opted not to present state-specific American Indian/Alaska Native counts and rates. However, in each of these cases, the aggregate national rates presented in this report include data for these registries.

\section{Methods for Identifying Cancer}

Medical facilities such as hospitals, doctors' offices, and pathology laboratories send information about cancer cases to their cancer registry. Most information comes from hospitals, where highly trained cancer registrars transfer the information from the patient's medical record to the registry's computer software using standardized codes. The data are then sent to the central cancer registry. Every year the central cancer registries electronically submit incidence, demographic, and clinical data to NPCR or SEER.

\section{Population Coverage}

The population coverage for incidence data varies by diagnosis year. Population coverage might be affected by the suppression of state incidence data, if a state did not meet the publication criteria or did not submit data. In addition, state incidence data might be suppressed if $<16$ cases were reported or if the state requested that the data be suppressed. Additional information is provided by the USCS technical notes (http:// www.cdc.gov/cancer/npcr/uscs/pdf/uscs-2012-technical-notes. pdf\#nameddest $=$ CensusRegionPubCriteria). Mortality data from malignant neoplasms (i.e., cancers) as recorded in the NVSS from the 50 states and DC are available in USCS, and thus $100 \%$ of the U.S. population is covered each year. However, state death data might be suppressed if $<16$ deaths were reported.

\section{Suppression of Rates and Counts}

When the numbers of cases or deaths used to compute rates are small, those rates tend to have poor reliability. Therefore, in an effort to discourage misinterpretation or use of rates or counts that are unstable because case or death counts are small, incidence and death rates and counts of $<16$ are not shown in tables and figures. The use of a threshold value for suppressing cells helps protect the confidentiality of patients by reducing or eliminating the risk for disclosure of their identity. Additional information is provided in the USCS technical notes (http:// www.cdc.gov/cancer/npcr/uscs/pdf/uscs-2012-technical-notes. pdf\#nameddest=Suppression).

\section{Publication Criteria}

Cancer incidence data that appear in this report are derived from state cancer registries that have high-quality cancer incidence data for individual (e.g., 2012) and combined (e.g., 1999-2012) years as demonstrated by meeting all of the following criteria on data quality for all cancer sites combined:

- case ascertainment is $\geq 90 \%$ (margin of error $+5 \%$ ) complete,

- $\leq 5 \%$ of cases are ascertained solely on the basis of a death certificate,

- $\leq 3 \%$ of cases are missing information on sex,

- $\leq 3 \%$ of cases are missing information on age,

- $\leq 5 \%$ of cases are missing information on race, and

- $\geq 97 \%$ of the registry's records passed a set of single-field and inter-field computerized edits that test the validity and logic of data components.

Additional information about USCS is available at http://www.cdc.gov/uscs.

\section{Highlights}

\section{Incidence and Death Rates}

In 2012, approximately 1.5 million invasive cancers were diagnosed in the United States, an annual incidence rate of 440 cases per 100,000 persons (Table 1 ). In the same year, 
approximately 582,600 persons died of cancer nationally, an annual death rate of 166 deaths per 100,000 persons (Table 2). By state, overall (all cancer sites combined) cancer incidence rates in 2012 ranged from 371 to 515 cases per 100,000 persons (Table 3), and overall cancer death rates ranged from 129 to 201 deaths per 100,000 persons (Table 4).

Cancer incidence (Table 5) and death (Table 6) rates increase with age. In 2012, among persons in the youngest age group ( $<15$ years), 9,967 new cancer cases (rate: 17 cases per 100,000 persons) and 1,367 cancer deaths (rate: two deaths per 100,000 persons) were reported. Among persons aged $\geq 65$ years, 826,841 new cancer cases (rate: 1,933 cases per 100,000 persons) and 403,497 cancer deaths (rate: 935 deaths per 100,000 persons) were reported. Overall, $54 \%$ of cancer cases and $69 \%$ of cancer deaths in 2012 occurred among persons aged $\geq 65$ years.

Overall and for many cancer sites, males had higher incidence (Table 7) and death rates (Table 8) than did females. In 2012, blacks had the highest cancer incidence (Table 9) and death (Table 10) rates in the United States, and American Indians/ Alaska Natives and Asians/Pacific Islanders had the lowest cancer incidence and death rates. Overall and for most cancer sites, Hispanics had lower cancer incidence (Table 11) and death rates (Table 12) than did non-Hispanics. Differences in cancer rates by race and ethnicity (Figure 1) might reflect differences in risk factors, screening, and treatment although rates among some populations might be underestimated because of problems ascertaining race or ethnicity.

By state and site, cancer incidence rates in 2012 ranged from 70 to 157 per 100,000 males for prostate cancer, 107 to 141 per 100,000 females for breast cancer, 4 to 10 per 100,000 females for cervical cancer, 29 to 92 per 100,000 persons for lung cancer, and 30 to 49 per 100,000 persons for colorectal cancer (Figure 2). By state and site, cancer death rates in 2012 ranged from 13 to 32 per 100,000 males for prostate cancer, 16 to 31 per 100,000 females for breast cancer, 1 to 5 per 100,000 females for cervical cancer, 20 to 69 per 100,000 persons for lung cancer, and 11 to 19 per 100,000 persons for colorectal cancer (Figure 3).

Differing rates of cancer by race, ethnicity, and state of residence indicate that for some populations, Healthy People 2020 objectives have already been achieved, whereas objectives for other populations have not been met and these populations might benefit from targeted cancer prevention and control efforts.

Four cancer sites accounted for $49 \%$ of all cases diagnosed in 2012, including 224,147 female breast cancers, 210,828 lung and bronchus cancers (111,395 among men and 99,433 among women), 177,489 prostate cancers, and 134,784 colon and rectum cancers (70,204 among men and 64,580 among women) (Table 13). These four sites also accounted for $48 \%$ of cancer deaths in 2012, including 157,423 lung cancer deaths, 51,516 colon and rectum cancer deaths, 41,150 female breast cancer deaths, and 27,244 prostate cancer deaths (Table 14).

\section{Time Trends in Incidence and Death Rates}

On the basis of data from registries meeting data quality criteria during 1999-2012, cancer incidence rates declined from 484 cancer cases per 100,000 population in 1999 to 434 cases in 2012 (Table 15). Although lung cancer incidence declined steadily among men from 1999 to 2012, it increased among women from 1999 to 2005 and has since declined from 2005 to 2012. Prostate cancer incidence declined from 170 cases per 100,000 men in 1999 to 105 cases in 2012. Colorectal cancer incidence declined from 56 cases per 100,000 persons in 1999 to 39 cases in 2012. Female breast cancer incidence declined from 135 cases per 100,000 women in 1999 to 121 cases in 2005, increased to 125 cases in 2009, and declined again to 122 cases in 2012. Time trends in cancer incidence rates are presented by cancer site, sex, and race (Figure 4) and by cancer site, sex, and ethnicity (Figure 5). During 1999-2012, cancer death rates declined from 201 deaths per 100,000 persons in 1999 to 166 deaths in 2012; during the same period, death rates declined for each of the four most common cancers (Table 16). Time trends in cancer death rates are presented by cancer site, sex, and race (Figure 6) and by cancer site, sex, and ethnicity (Figure 7).

National cancer surveillance data help public health officials track progress toward achieving the national cancer objectives set forth in Healthy People 2020 (20). For the national cancer burden to be reduced and Healthy People 2020 targets to be met, behavioral and environmental factors that increase cancer risk must be reduced, and high-quality screening services, timely follow-up, and evidence-based treatments must be available and accessible to all persons. Several effective primary and secondary prevention measures, such as vaccination against infectious agents that cause cancer (i.e., hepatitis B virus and human papillomavirus), help with smoking cessation, and cancer screening, when effectively implemented and sustained, could reduce the number of new cancer cases and prevent many cancer-related deaths (21). Evidence-based interventions can be implemented at both the individual level and the population level to reduce cancer risk factors, promote healthy living, and encourage cancer screening (5).

\section{Acknowledgment}

Data were provided by state and regional cancer registry personnel. 


\section{References}

1. US Cancer Statistics Working Group. United States cancer statistics: 1999-2012. Incidence and mortality web-based report. Atlanta, GA: US Department of Health and Human Services, CDC, National Cancer Institute; 2015. http://www.cdc.gov/uscs.index.htm

2. CDC. Summary of notifiable noninfectious conditions and disease outbreaks_-United States. MMWR Morb Mortal Wkly Rep 2014;63(55).

3. CDC. Summary of notifiable infectious diseases and conditions-United States, 2014. MMWR Morb Mortal Wkly Rep 2014;63(54).

4. Heron M. Deaths: leading causes for 2012. Natl Vital Stat Rep 2015;64:193. http://www.cdc.gov/nchs/data/nvsr/nvsr64/nvsr64_10.pdf

5. Colditz GA, Wolin KY, Gehlert S. Applying what we know to accelerate cancer prevention. Sci Transl Med 2012;4:127rv4. http://dx.doi. org/10.1126/scitranslmed.3003218

6. Weir HK, Thun MJ, Hankey BF, et al. Annual report to the nation on the status of cancer, 1975-2000, featuring the uses of surveillance data for cancer prevention and control. J Natl Cancer Inst 2003;95:1276-99. http://dx.doi.org/10.1093/jnci/djg040

7. US Department of Health and Human Services. Healthy people 2020. Washington, DC: US Department of Health and Human Services; 2011. http://www.healthypeople.gov/2020/topicsobjectives2020/default.aspx

8. Fisher R, Haenlein M. Legislative authorizations for cancer registries. In: National Cancer Institute, National Institutes of Health. State cancer legislative database update. Bethesda, MD: US Department of Health and Human Services, Public Health Service, National Institutes of Health. National Cancer Institute; 1991:8-15.

9. CDC. State cancer registries: status of authorizing legislation and enabling regulations-United States, October 1993. MMWR Morb Mortal Wkly Rep 1994;43:71-5.

10. Fritz ARL. The SEER program code manual. Bethesda, MD: National Cancer Institute; 1998.

11. Havener LTM, editor. Standards for cancer registries. Volume II: data standards and data dictionary. 13th ed. Version 11.3. Springfield, IL: North American Association of Central Cancer Registries; 2008.
12. Fritz A, Percy C, Jack A. International classification of diseases of oncology. Geneva, Switzerland: World Health Organization; 2000.

13. National Cancer Institute. Hematopoietic codes based on WHO classification of tumours of haematopoietic and lymphoid tissues. Rockville, MD: US Department of Health and Human Services, National Cancer Institute; 2008. http://seer.cancer.gov/iccc

14. Hetzel AMUS. Vital Statistics System: major activities and developments, 1950-95. Atlanta, GA: US Department of Health and Human Services, CDC, National Center for Health Statistics; 1997. PHS 97-1993.

15. World Health Organization. International statistical classification of diseases and related health problems. 10th revision. Geneva, Switzerland: World Health Organization; 1992.

16. Miniño AM, Heron MP, Murphy SL, Kochanek KD; CDC, National Center for Health Statistics, National Vital Statistics System. Deaths: final data for 2004. Natl Vital Stat Rep 2007;55:1-119.

17. Clegg LX, Reichman ME, Hankey BF, et al. Quality of race, Hispanic ethnicity, and immigrant status in population-based cancer registry data: implications for health disparity studies. Cancer Causes Control 2007;18:177-87. http://dx.doi.org/10.1007/s10552-006-0089-4

18. Arias E, Schauman WS, Eschbach K, Sorlie PD, Backlund E. The validity of race and Hispanic origin reporting on death certificates in the United States. Vital Health Stat 2 2008;(148):1-23. http://www.cdc.gov/nchs/ data/series/sr_02/sr02_148.pdf

19. NAACCR Asian Pacific Islander Work Group. NAACCR Asian Pacific Islander identification algorithm. Springfield, IL: North American Association of Central Cancer Registries; 2008.

20. Henley SJ, Singh S, King J, Wilson R, Ryerson B. Invasive cancer incidence-United States, 2010. MMWR Morb Mortal Wkly Rep 2014;63:253-9.

21. Agency for Healthcare Research and Quality. The guide to clinical preventive services, 2014. Rockville, MD: Agency for Healthcare Research and Quality; 2015. http://www.ahrq.gov/professionals/ clinicians-providers/guidelines-recommendations/guide/index.html 
FIGURE 1. Age-adjusted rate* of invasive ${ }^{\dagger}$ cancer cases for most common sites, by sex and race/ethnicity ${ }^{\S}$ United States, $2012^{\Uparrow}$
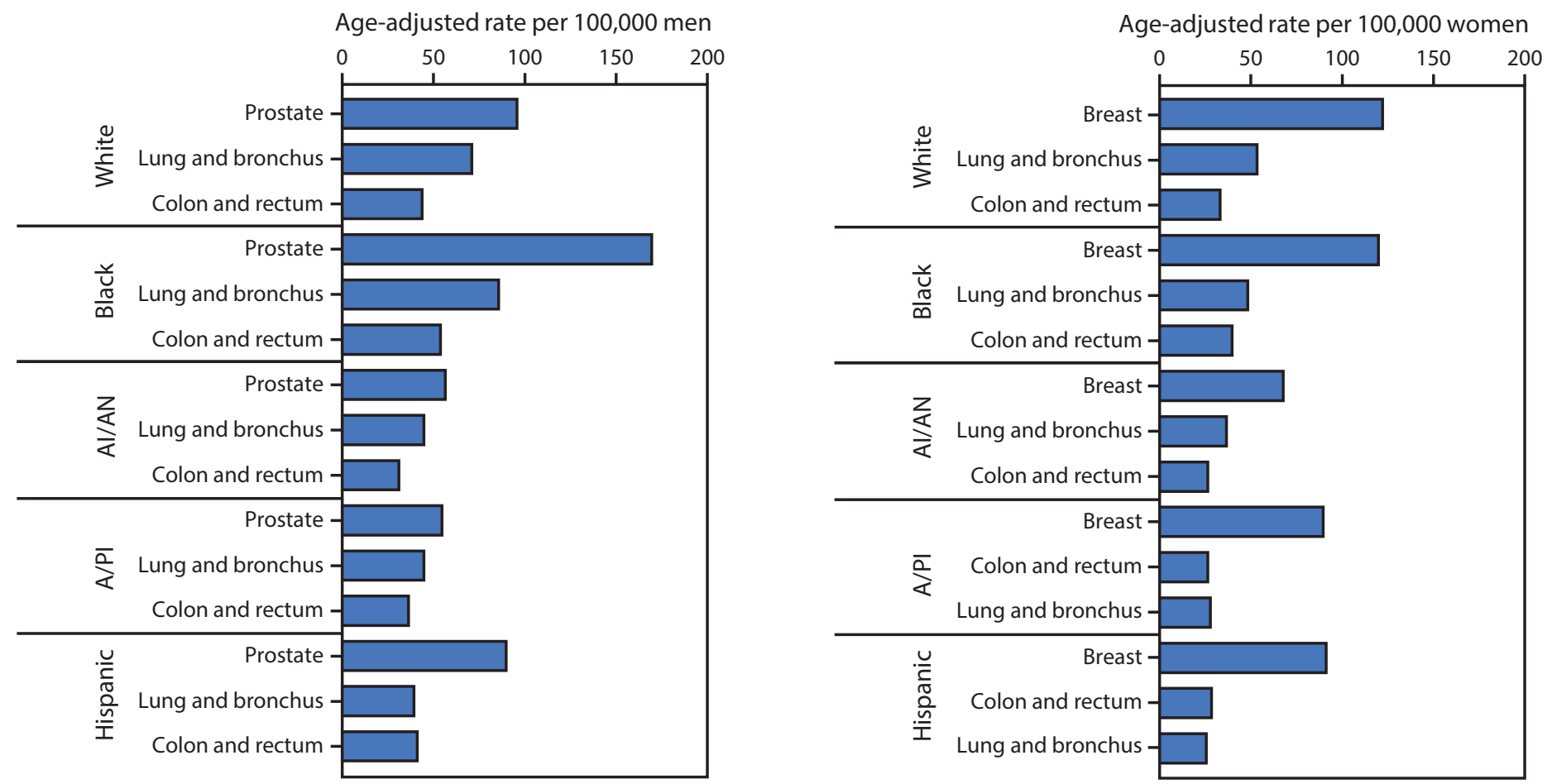

Abbreviations: Al/AN = American Indian/Alaska Native; A/PI = Asian/Pacific Islander.

Sources: CDC's National Program of Cancer Registries and National Cancer Institute's Surveillance, Epidemiology, and End Results program.

* Rates are the number of cases per 100,000 persons and are age-adjusted to the 2000 U.S. standard population (19 age groups - Census P25-1130). For more information, see USCS technical notes (http://www.cdc.gov/cancer/npcr/uscs/pdf/uscs-2012-technical-notes.pdf).

+ Invasive cancer excludes basal and squamous cell carcinomas of the skin except when these occur on the skin of the genital organs, and in situ cancers except urinary bladder. Urinary bladder cancer includes invasive and in situ.

$\S$ Race categories are not mutually exclusive from Hispanic origin. Rates are not presented for persons of unknown or other race. Data for specified racial or ethnic populations other than white and black should be interpreted with caution. For more information, see USCS technical notes (http://www.cdc.gov/cancer/npcr/uscs/ pdf/uscs-2012-technical-notes.pdf\#nameddest=IntRaceEthnicityData).

I" Data are compiled from cancer registries that meet the data quality criteria for all invasive cancer sites combined (covering approximately $99 \%$ of the U.S. population). Registry-specific data quality information is available at http://www.cdc.gov/cancer/npcr/uscs/pdf/uscs-2012-technical-notes.pdf\#nameddest=RegistriesPubCriteria. Caution should be used when comparing incidence and death rates because of the difference in population coverage. 


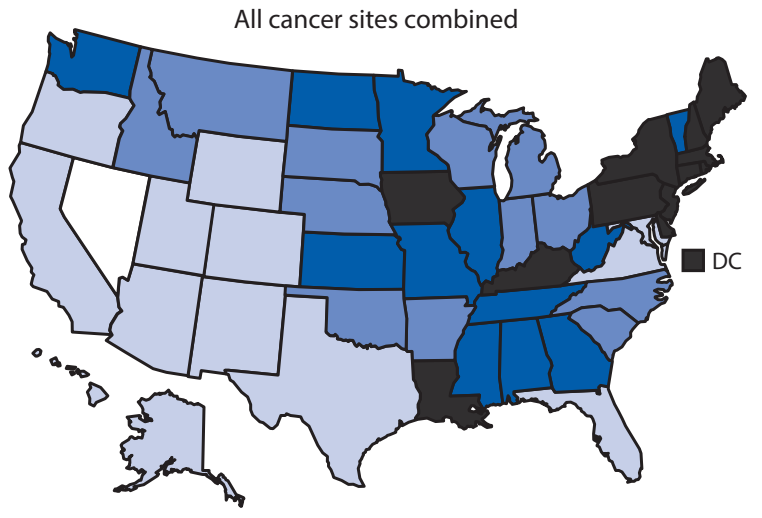

$\square$ 371.4-431.3 $\square 431.4-448.4 \square$ 448.5-463.0 $\square$ 463.1-515.1 $\square$ Data not available

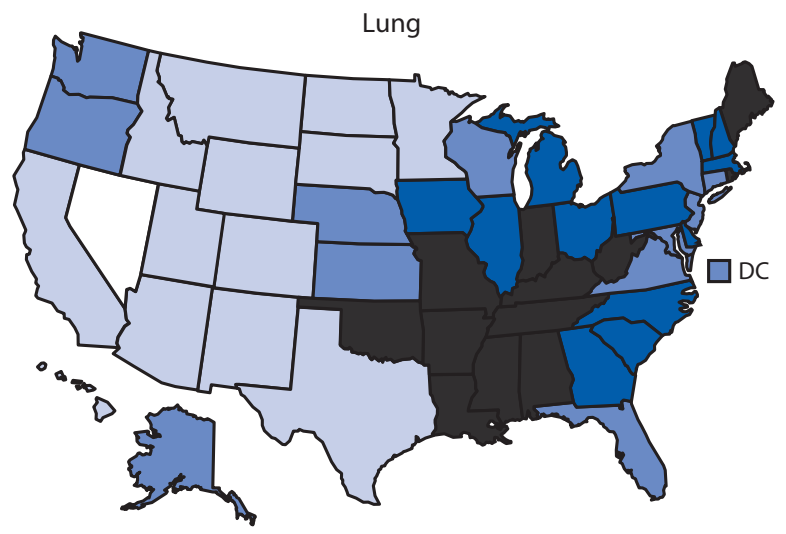

$\square 29.0-55.5$

$55.6-61.2$

61.3-67.9

68.0-92.4

Data not available

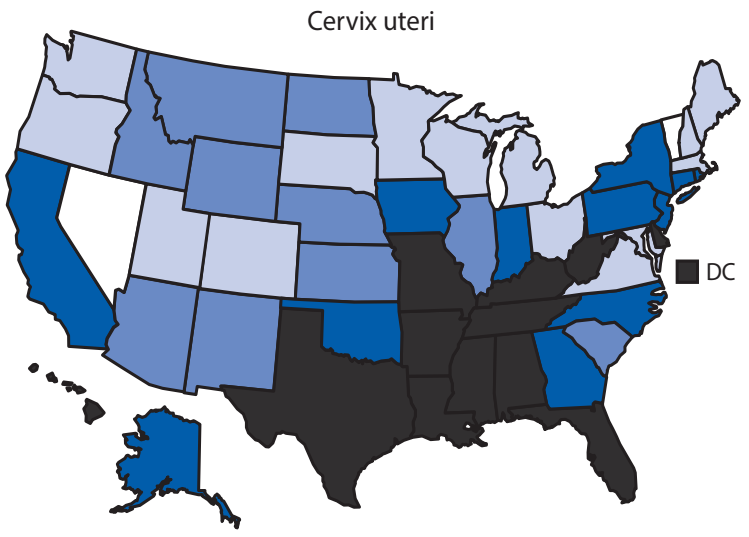

$\square 4.3-6.4$

$\square 6.5-7.0$

7.1-8.1

8.2-9.6

$\square$ Data not available

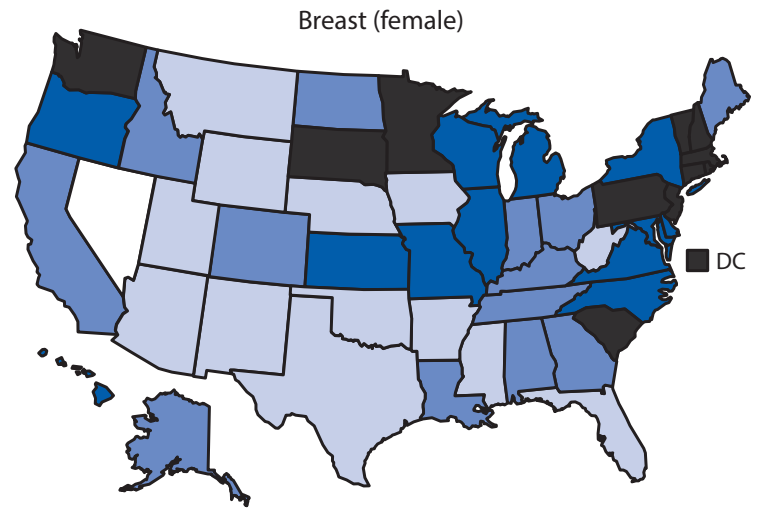

$\square$ 107.2-118.3 $\square$ 118.4-123.6 $\square$ 123.7-126.8 $\square$ 126.9-141.4 $\square$ Data not available

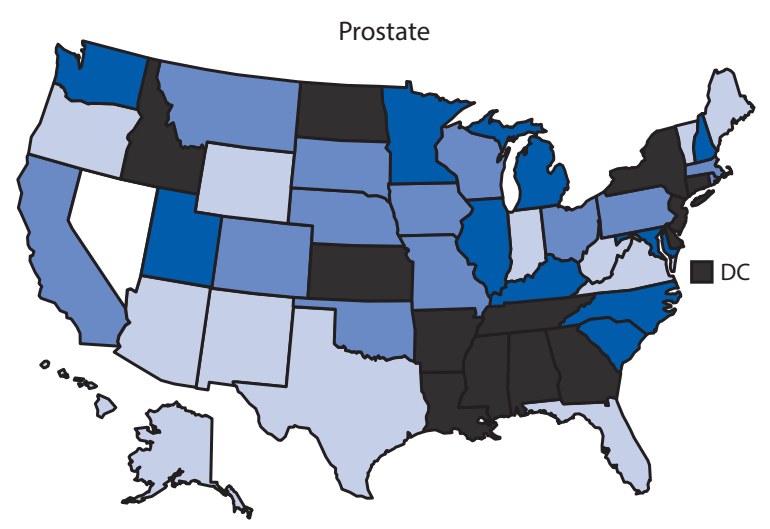

$\square$ 69.9-95.1 $\square 95.2-106.3 \square 106.4-114.8 \square 114.9-157.1 \quad \square$ Data not available

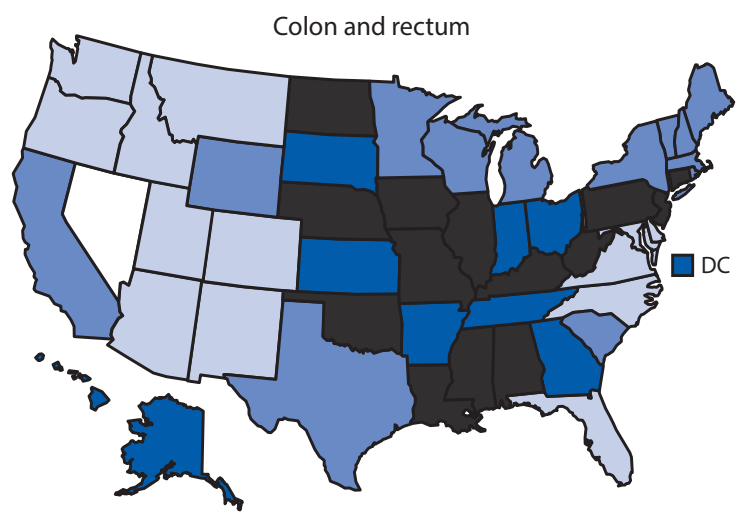

$\square$ 30.2-36.4 $\square 36.5-39.0 \quad \square 39.1-42.3 \quad \square$ 42.4-49.2 $\square$ Data not available

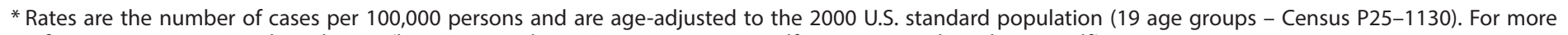
information, see USCS technical notes (http://www.cdc.gov/cancer/npcr/uscs/pdf/uscs-2012-technical-notes.pdf).

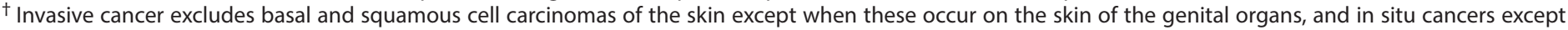
urinary bladder. Urinary bladder cancer includes invasive and in situ.

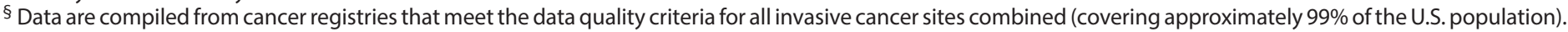

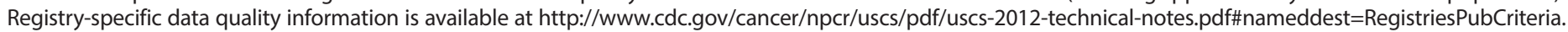
Caution should be used when comparing incidence and death rates because of potential differences in population coverage. 
FIGURE 3. Age-adjusted rate* of cancer deaths, by primary cancer site and state - United States, $2012^{\dagger}$
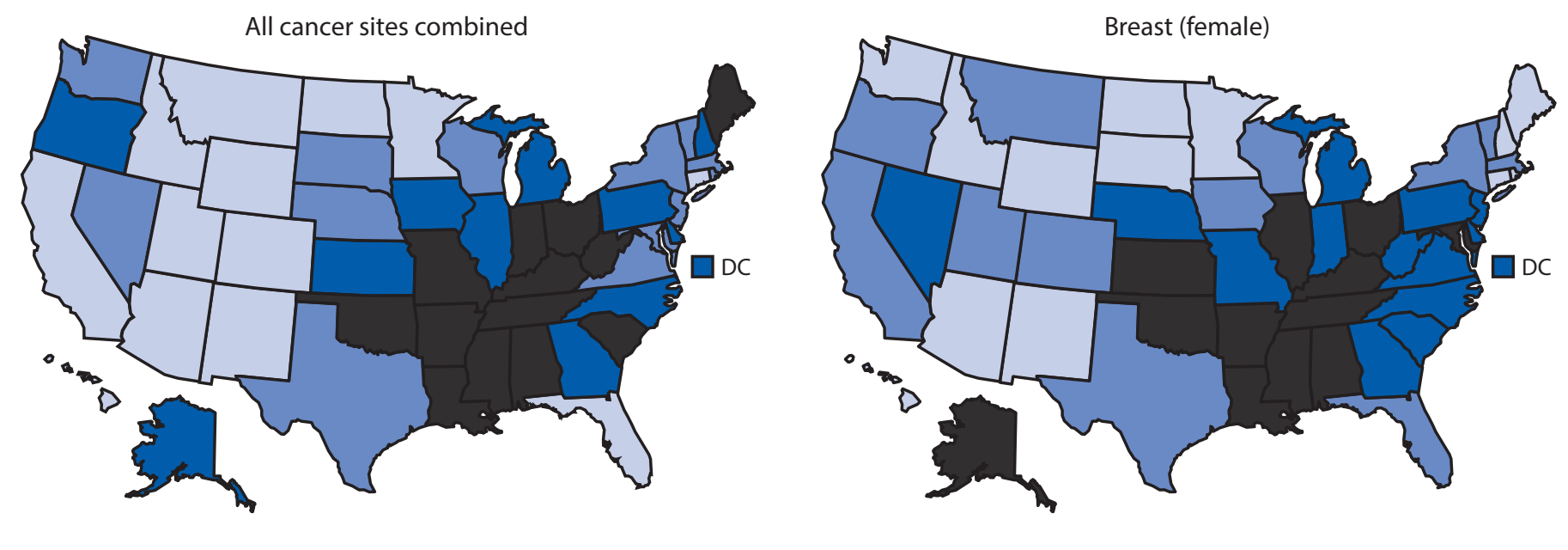

$\square 128.7-158.6 \square 158.7-166.1 \square 166.2-178.7 \square 178.8-201.2$

$19.2-21.1$

$21.2-22.6$

$22.7-31.1$
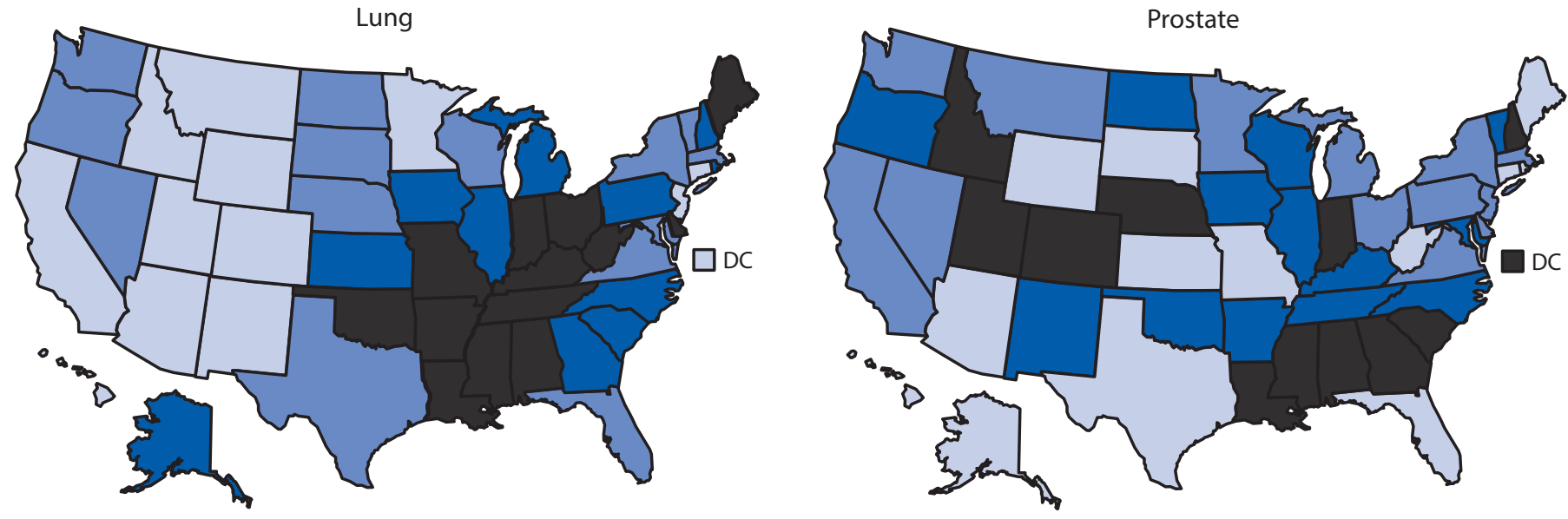

$\square 20.4-40.5 \quad \square 40.6-46.1$

$46.2-52.1$

$52.2-68.8$

Cervix uteri

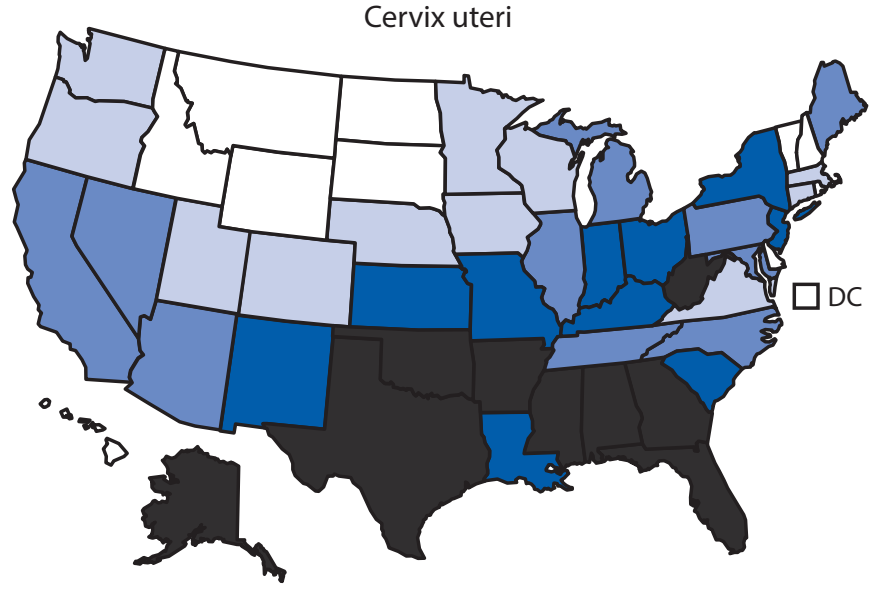

$\square$ 1.1-1.9 $\square$ 2.0-2.2 $\square$ 2.3-2.7 $\square$ 2.8-5.3 $\square$ Data not available

$20.1-21.3$

$21.4-31.8$

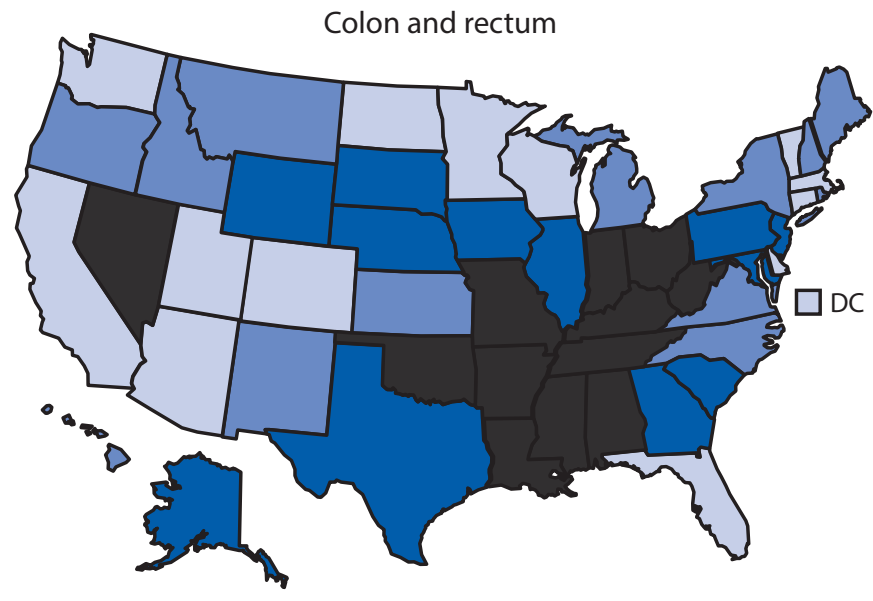

$\square 10.6-13.6 \quad \square 13.7-14.4 \quad \square 14.5-15.9 \quad \square 16.0-19.2$

* Rates are the number of deaths per 100,000 persons and are age-adjusted to the 2000 U.S. standard population (19 age groups - Census P25-1130). For more information, see USCS Technical notes (http://www.cdc.gov/cancer/npcr/uscs/pdf/uscs-2012-technical-notes.pdf).

${ }^{\dagger}$ Data are from the National Vital Statistics System (NVSS). Data for death rates cover $100 \%$ of the U.S. population. Caution should be used when comparing incidence and death rates because of potential differences in population coverage. 
FIGURE 4. Age-adjusted rate* of invasive ${ }^{\dagger}$ cancer cases, by primary cancer site, race, ${ }^{\S}$ and sex — United States, 1999-2012 ${ }^{\uparrow}$

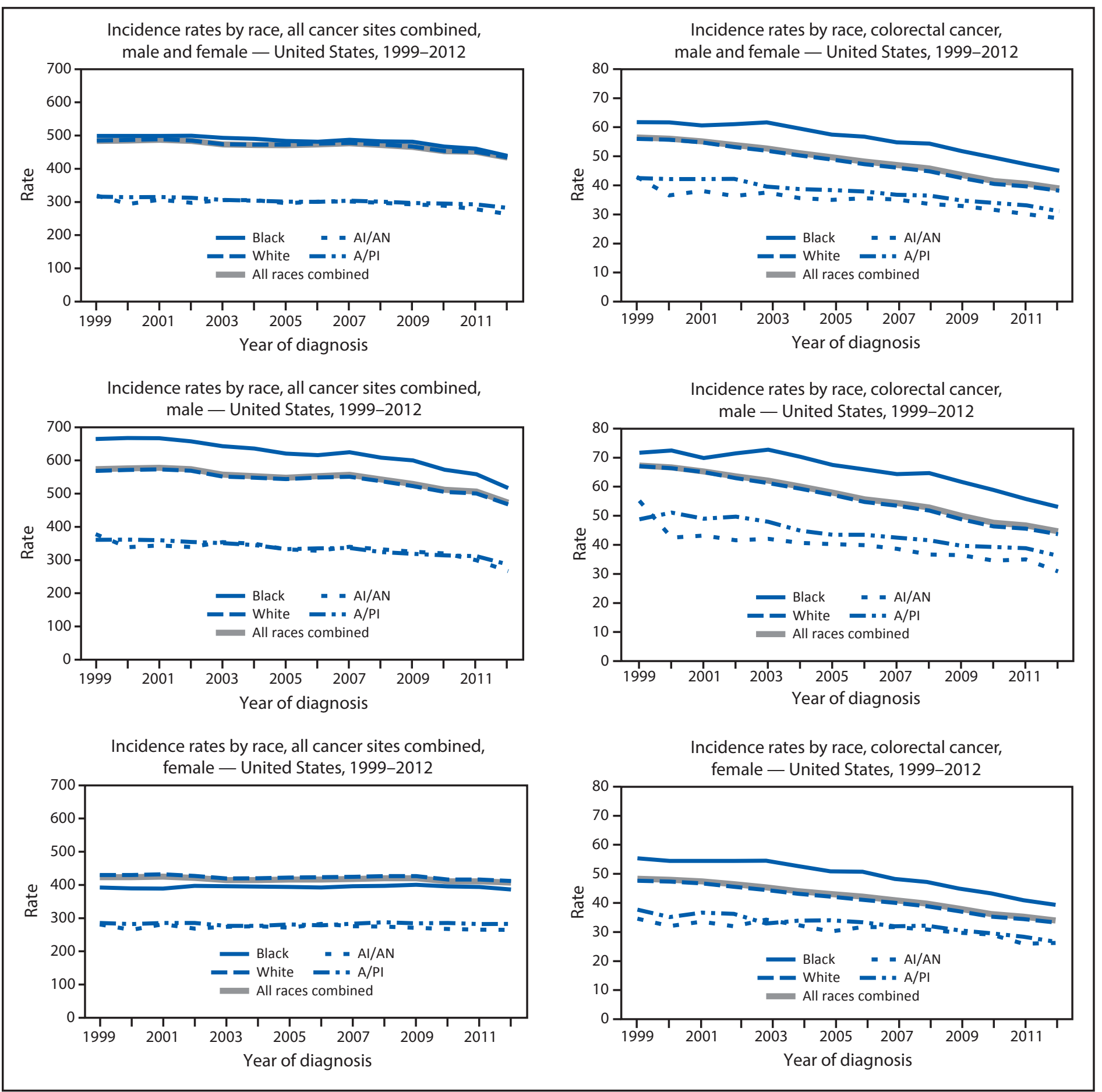

See figure footnotes on the next page. 
FIGURE 4. (Continued) Age-adjusted rate* of invasive ${ }^{\dagger}$ cancer cases, by primary cancer site, race, ${ }^{\S}$ and sex — United States, 1999-2012
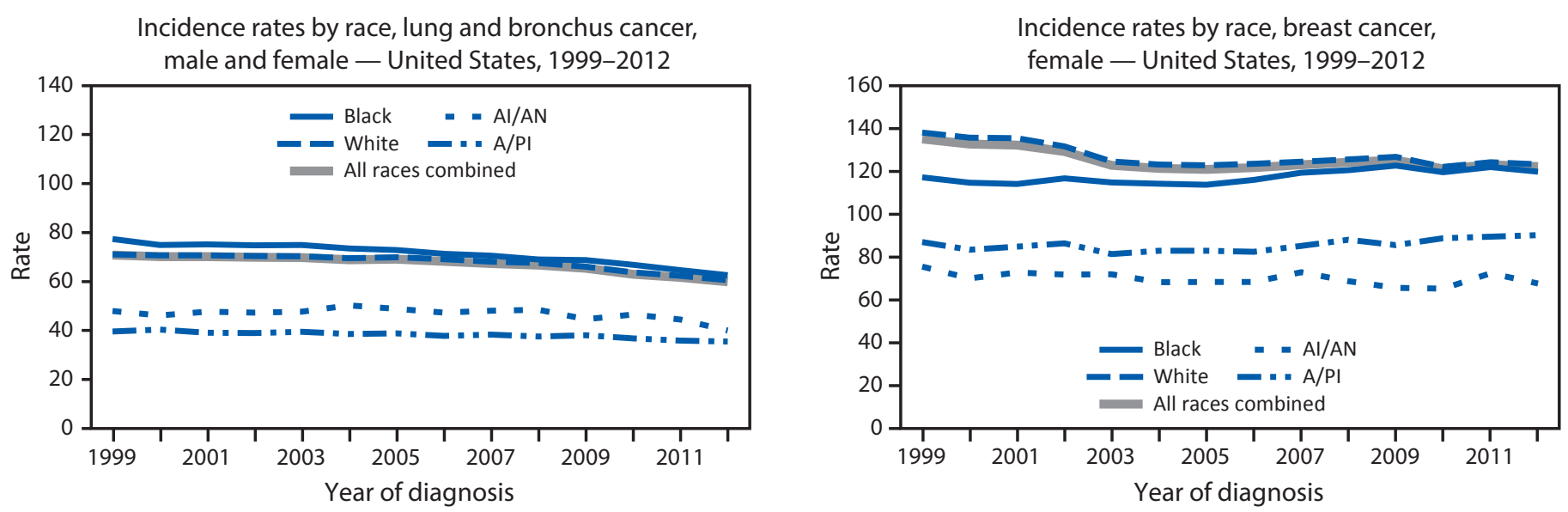

Incidence rates by race, lung and bronchus cancer, male - United States, 1999-2012
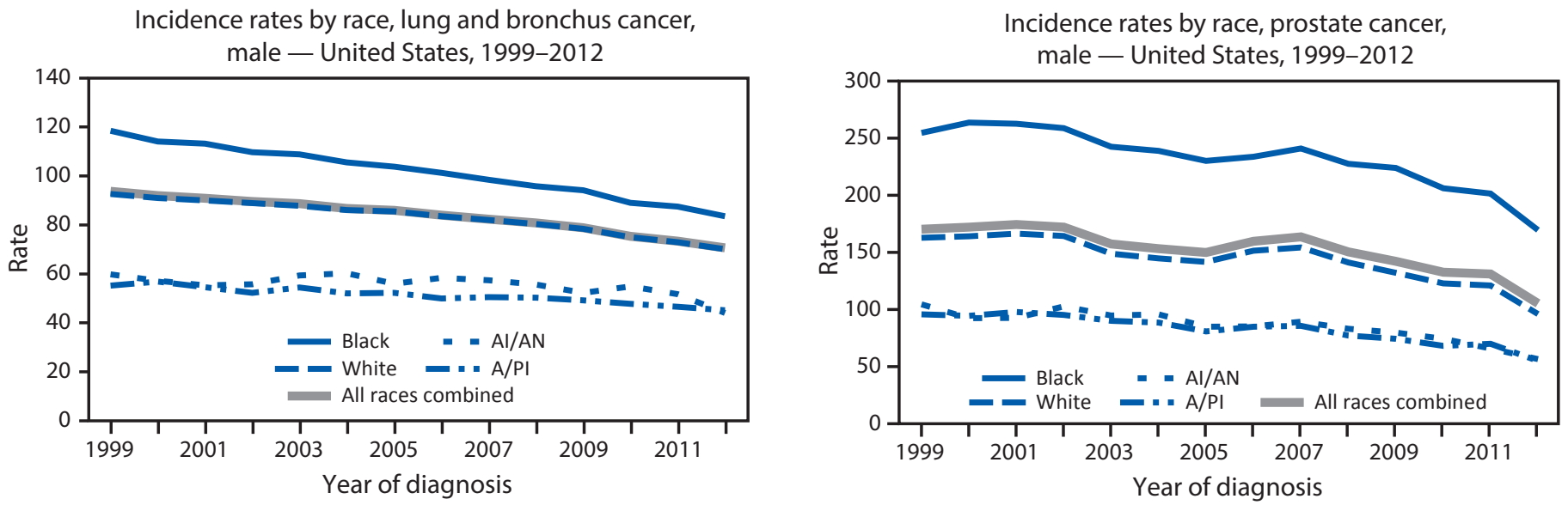

Incidence rates by race, lung and bronchus cancer, female - United States, 1999-2012

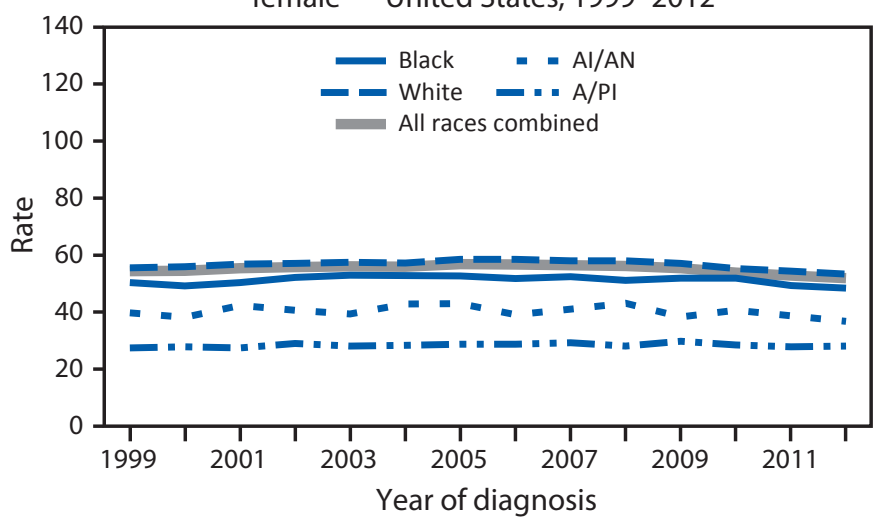

Abbreviations: Al/AN = American Indian/Alaska Native; A/PI = Asian/Pacific Islander.

* Rates are the number of cases per 100,000 persons and are age-adjusted to the 2000 U.S. standard population (19 age groups - Census P25-1130). For more information, see USCS technical notes (http://www.cdc.gov/cancer/npcr/uscs/pdf/uscs-2012-technical-notes.pdf).

+ Invasive cancer excludes basal and squamous cell carcinomas of the skin except when these occur on the skin of the genital organs, and in situ cancers except urinary bladder. Urinary bladder cancer includes invasive and in situ.

$\S$ Rates are not presented for persons of unknown or other race. Data for specified racial populations other than white and black should be interpreted with caution. For more information, see USCS technical notes (http://www.cdc.gov/cancer/npcr/uscs/pdf/uscs-2012-technical-notes.pdf\#nameddest=IntRaceEthnicityData).

I Data are compiled from cancer registries that meet the data quality criteria for all invasive cancer sites combined for all years, $1999-2012$ (covering approximately $92 \%$ of the U.S. population). See registry-specific data quality information for all years, 1999-2012 (http://www.cdc.gov/cancer/npcr/uscs/pdf/uscs-2012-technical-notes.pdf\#nameddest $=$ RegistriesPubCriteria). Caution should be used when comparing incidence and death rates because of potential differences in population coverage. 
FIGURE 5. Age-adjusted rate* of invasive ${ }^{\dagger}$ cancer cases, by primary cancer site, ethnicity, ${ }^{\S}$ and sex — United States, 1999-2012 ${ }^{\uparrow}$
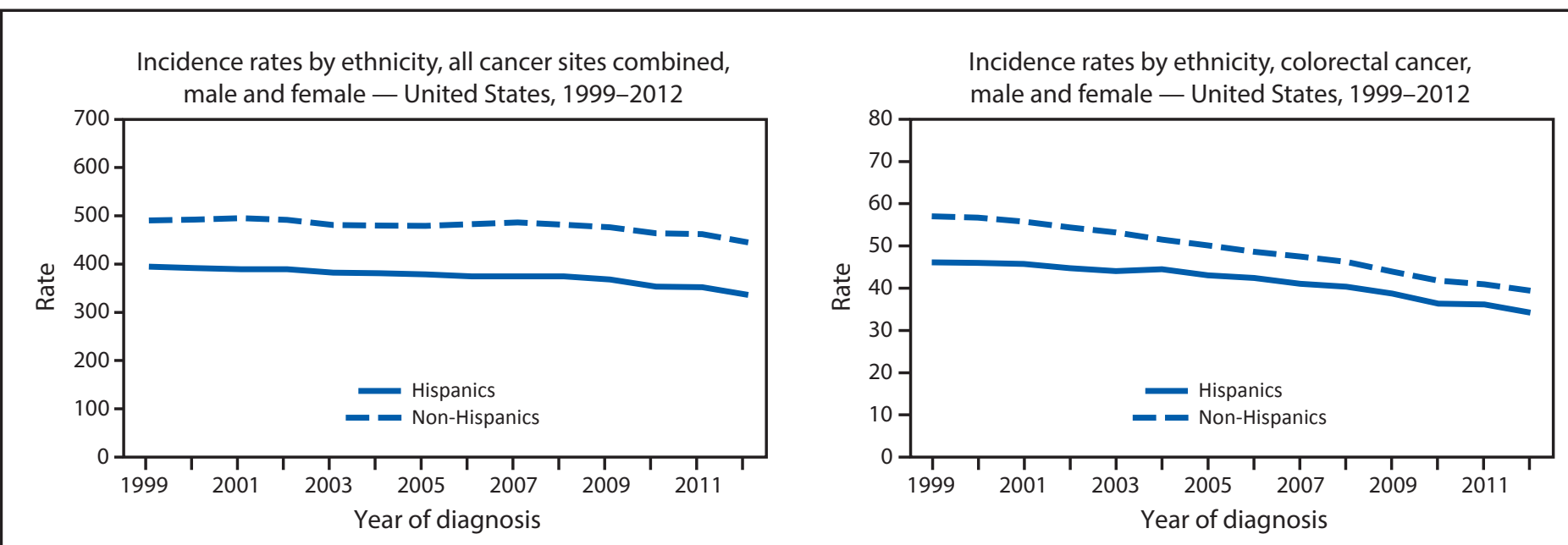

Incidence rates by ethnicity, all cancer sites combined, male — United States, 1999-2012
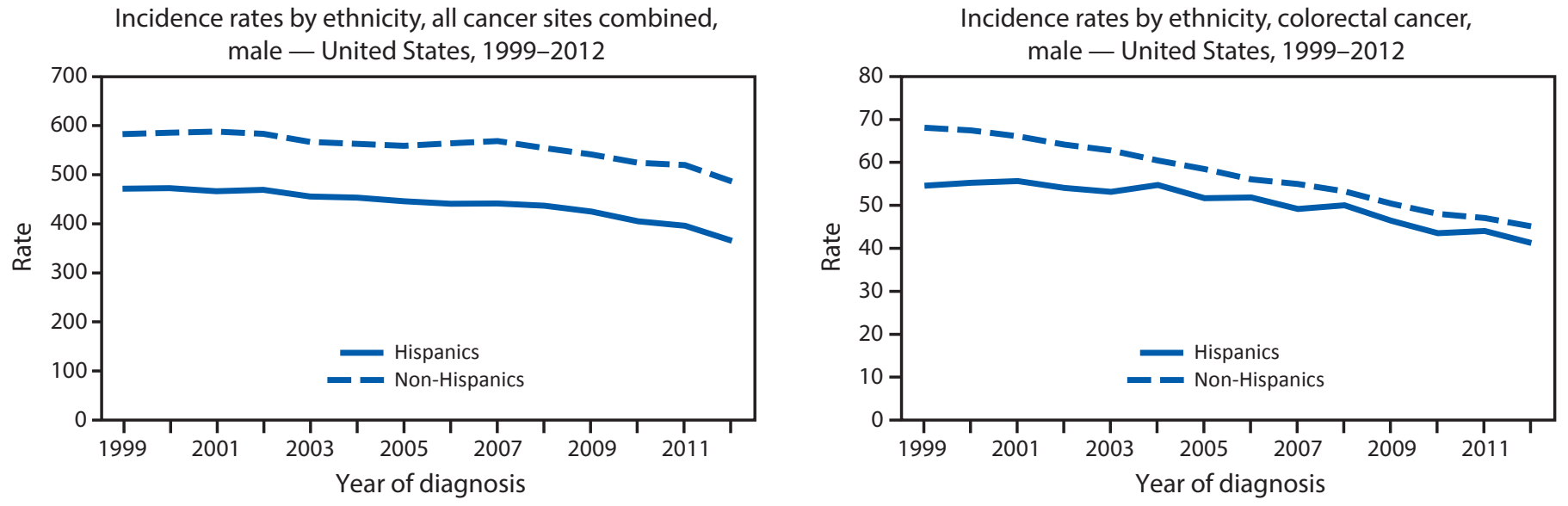

Incidence rates by ethnicity, all cancer sites combined, female - United States, 1999-2012
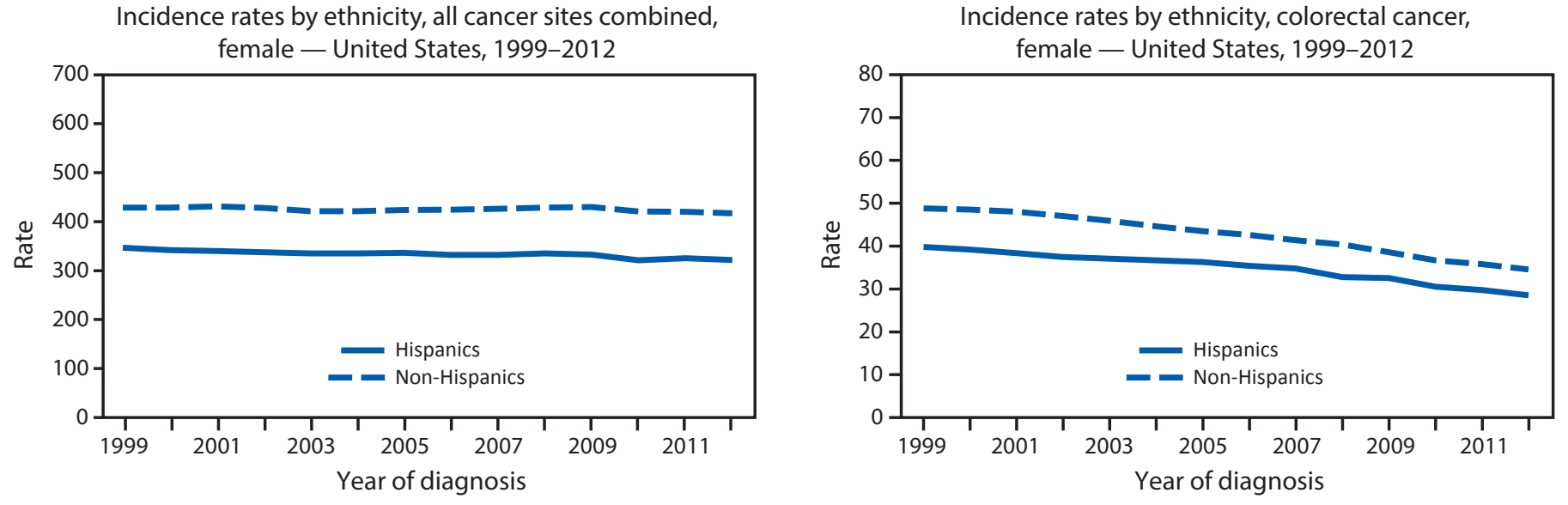

See figure footnotes on the next page. 
FIGURE 5. (Continued) Age-adjusted rate* of invasive ${ }^{\dagger}$ cancer cases, by primary cancer site, ethnicity, ${ }^{\S}$ and sex — United States, 1999-2012^

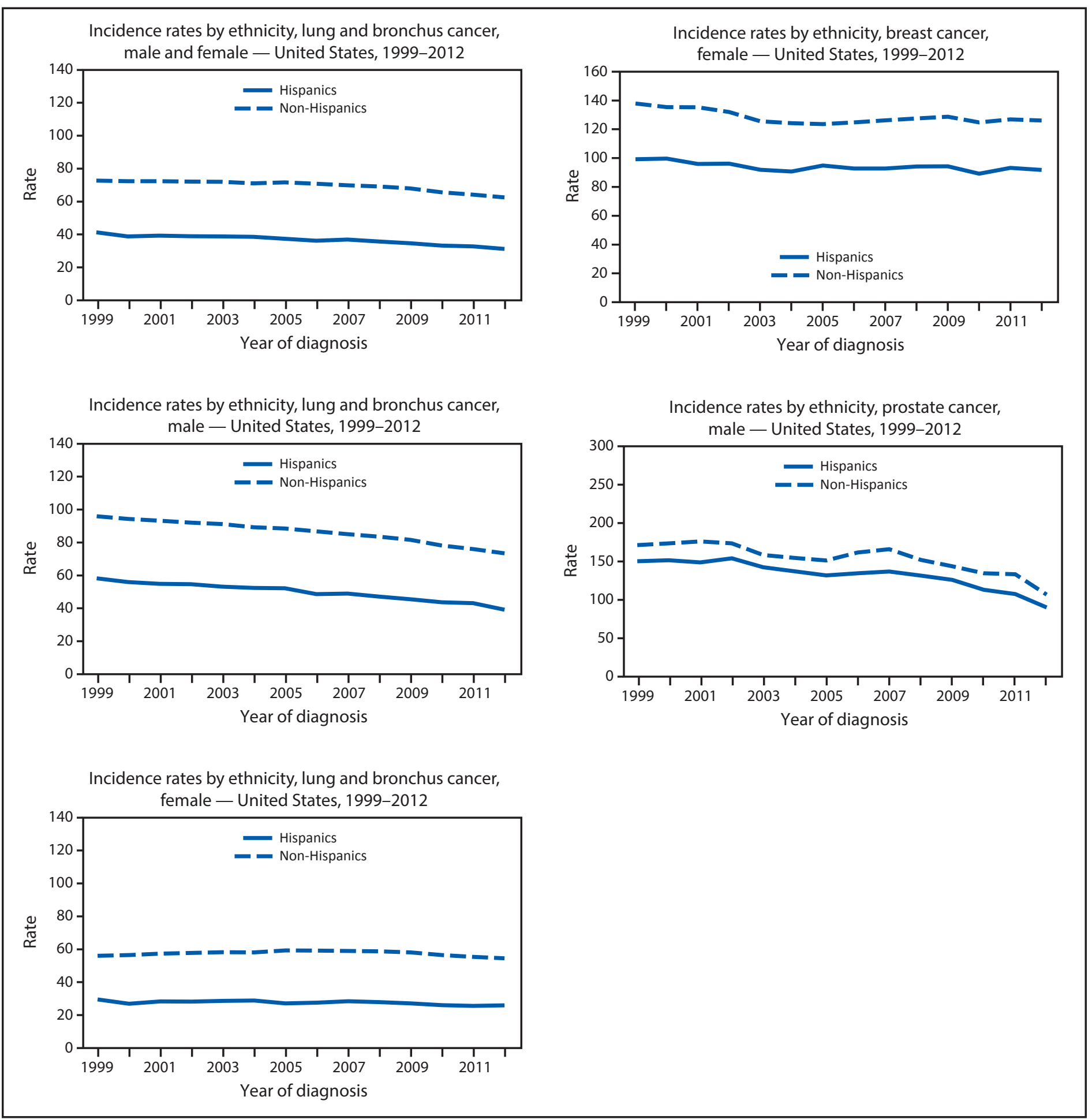

* Rates are the number of cases per 100,000 persons and are age-adjusted to the 2000 U.S. standard population (19 age groups - Census P25-1130). For more information, see USCS technical notes (http://www.cdc.gov/cancer/npcr/uscs/pdf/uscs-2012-technical-notes.pdf).

+ Invasive cancer excludes basal and squamous cell carcinomas of the skin except when these occur on the skin of the genital organs, and in situ cancers except urinary bladder. Urinary bladder cancer includes invasive and in situ.

$\S$ Data for specified ethnical populations should be interpreted with caution. For more information, see USCS technical notes (http://www.cdc.gov/cancer/npcr/uscs/ pdf/uscs-2012-technical-notes.pdf\#nameddest=IntRaceEthnicityData).

I Data are compiled from cancer registries that meet the data quality criteria for all invasive cancer sites combined for all years, $1999-2012$ (covering approximately $92 \%$ of the U.S. population). See registry-specific data quality information for all years, 1999-2012 (http://www.cdc.gov/cancer/npcr/uscs/pdf/uscs-2012-technical-notes.pdf\#na meddest=RegistriesPubCriteria). Caution should be used when comparing incidence and death rates because of potential differences in population coverage. 
FIGURE 6. Age-adjusted rate* of cancer deaths, by primary cancer site, race, ${ }^{\dagger}$ and sex — United States, 1999-2012 ${ }^{\S}$

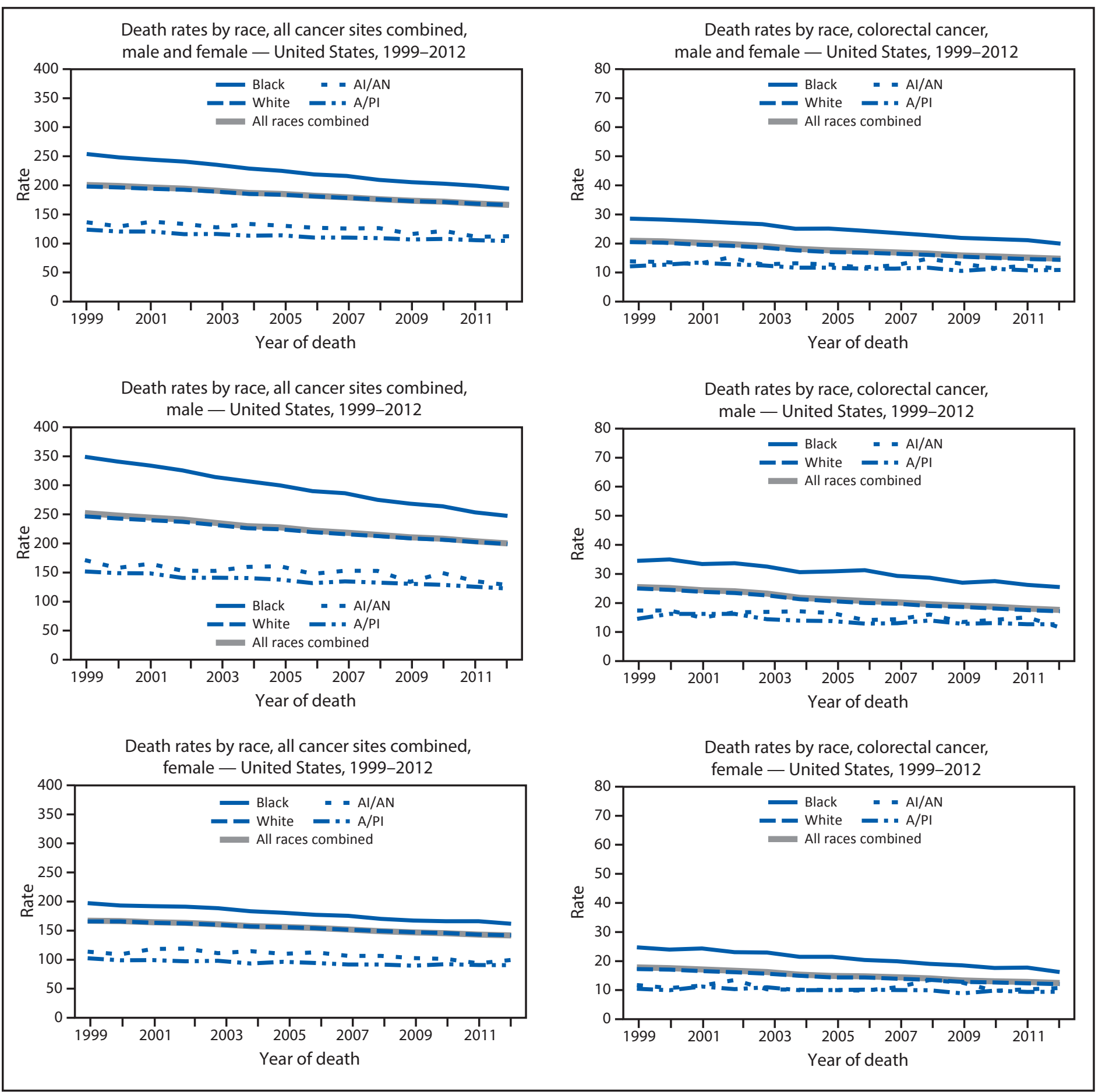

See figure footnotes on the next page. 
FIGURE 6. (Continued) Age-adjusted rate* of cancer deaths, by primary cancer site, race, ${ }^{\dagger}$ and sex — United States, 1999-2012§
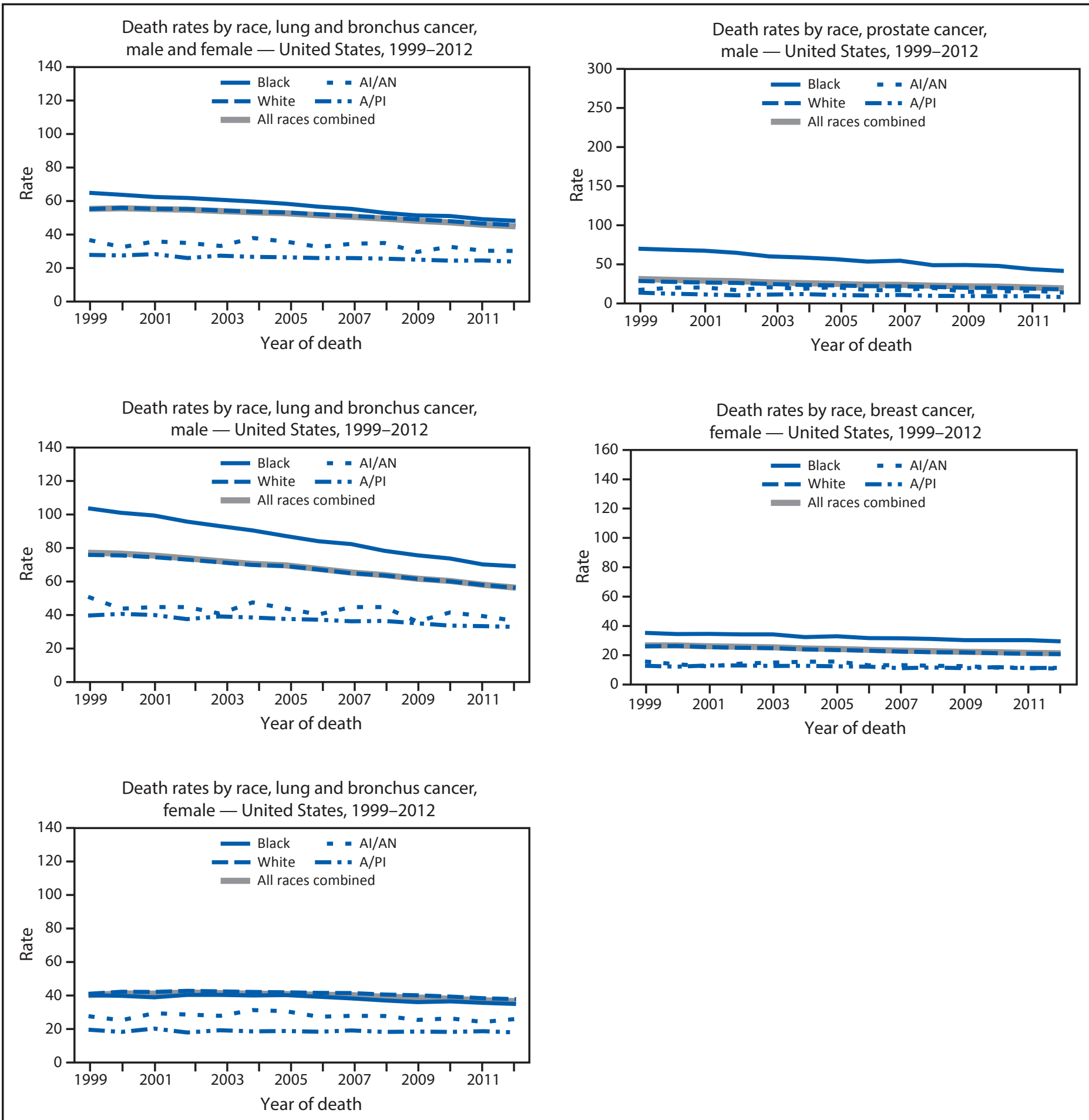

Abbreviations: Al/AN = American Indian/Alaska Native; A/PI = Asian/Pacific Islander.

* Rates are the number of deaths per 100,000 persons and are age-adjusted to the 2000 U.S. standard population (19 age groups - Census P25-1130). For more information, see USCS technical notes (http://www.cdc.gov/cancer/npcr/uscs/pdf/uscs-2012-technical-notes.pdf).

${ }^{\dagger}$ Rates are not presented for persons of unknown or other race. Data for specified racial populations other than white and black should be interpreted with caution. For more information, see USCS technical notes (http://www.cdc.gov/cancer/npcr/uscs/pdf/uscs-2012-technical-notes.pdf\#nameddest=IntRaceEthnicityData).

$\S$ Data are from the National Vital Statistics System (NVSS). Data for death rates cover $100 \%$ of the U.S. population. Caution should be used when comparing incidence and death rates because of potential differences in population coverage. 
FIGURE 7. Age-adjusted rate* of cancer deaths, by primary cancer site, ethnicity, ${ }^{\dagger}$ and sex — United States, 1999-2012 ${ }^{\S}$
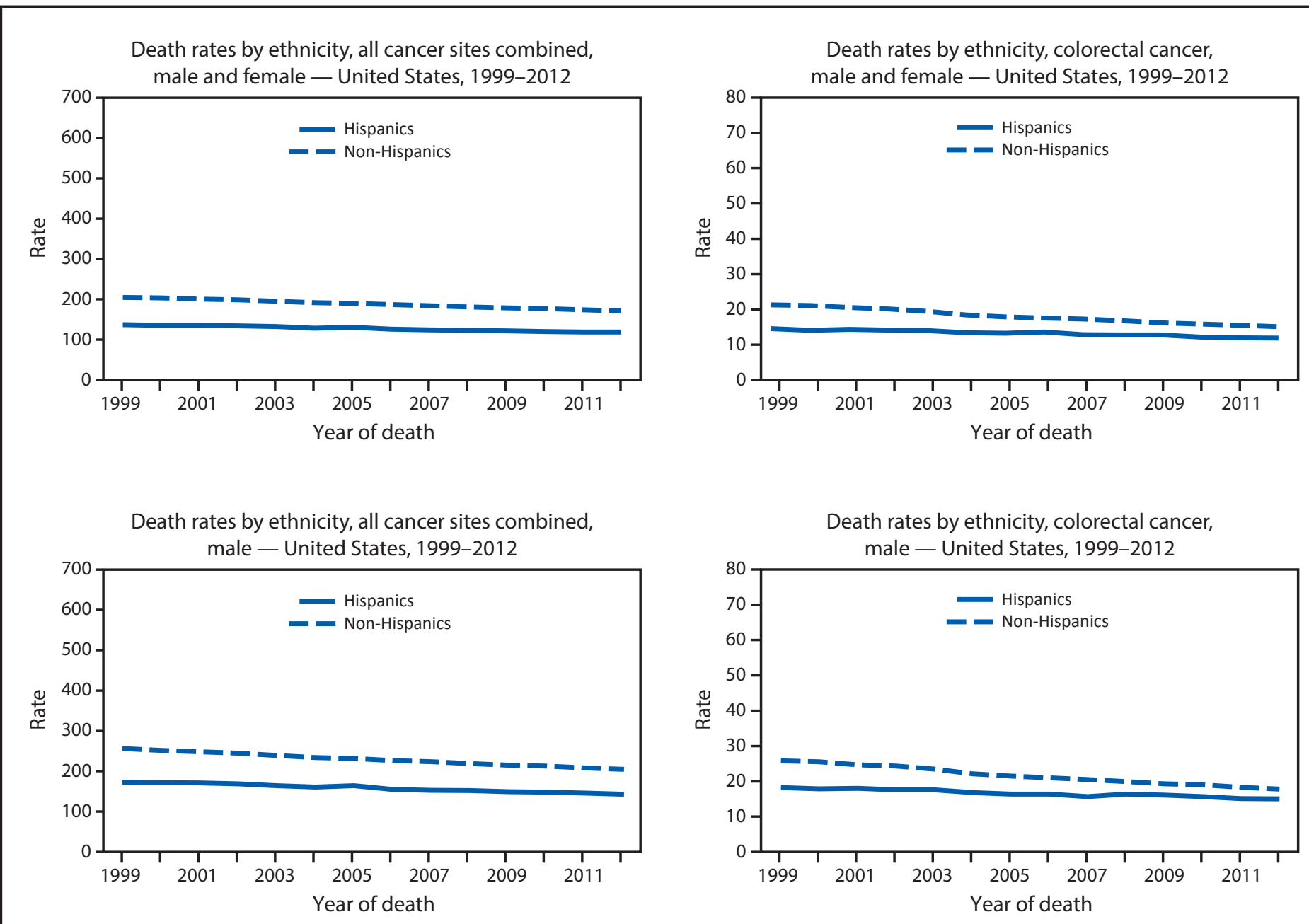

Death rates by ethnicity, all cancer sites combined, female - United States, 1999-2012
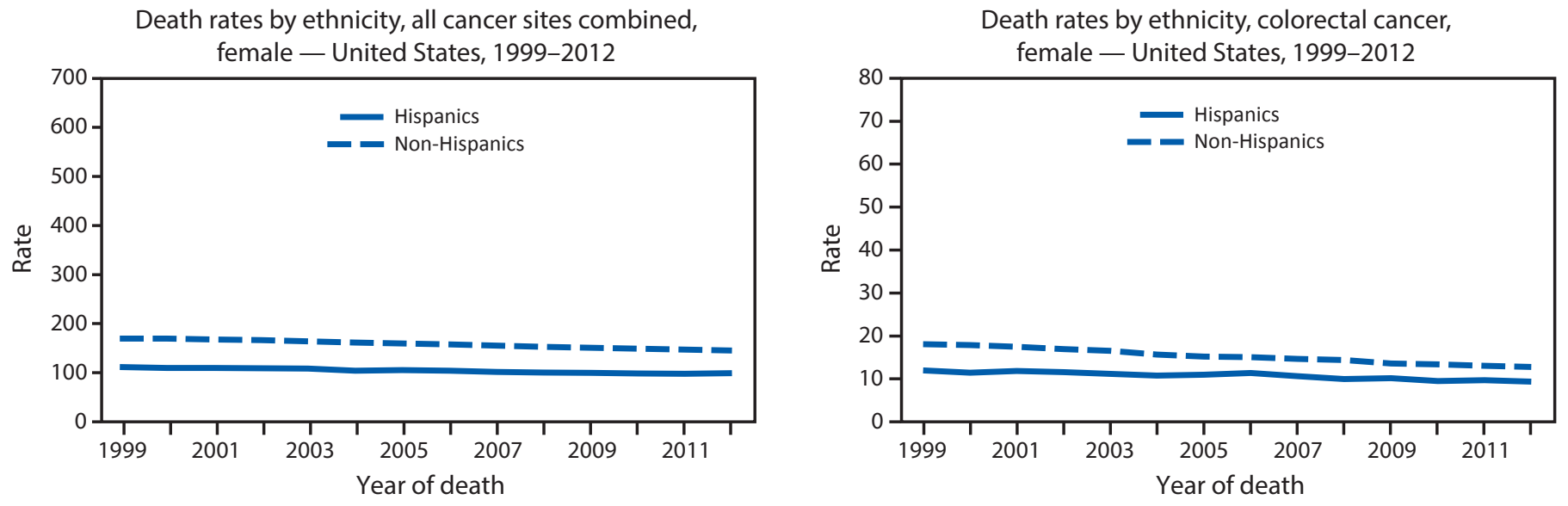

See figure footnotes on the next page. 
FIGURE 7. (Continued) Age-adjusted rate* of cancer deaths, by primary cancer site, ethnicity, ${ }^{\dagger}$ and sex — United States, 1999-2012§

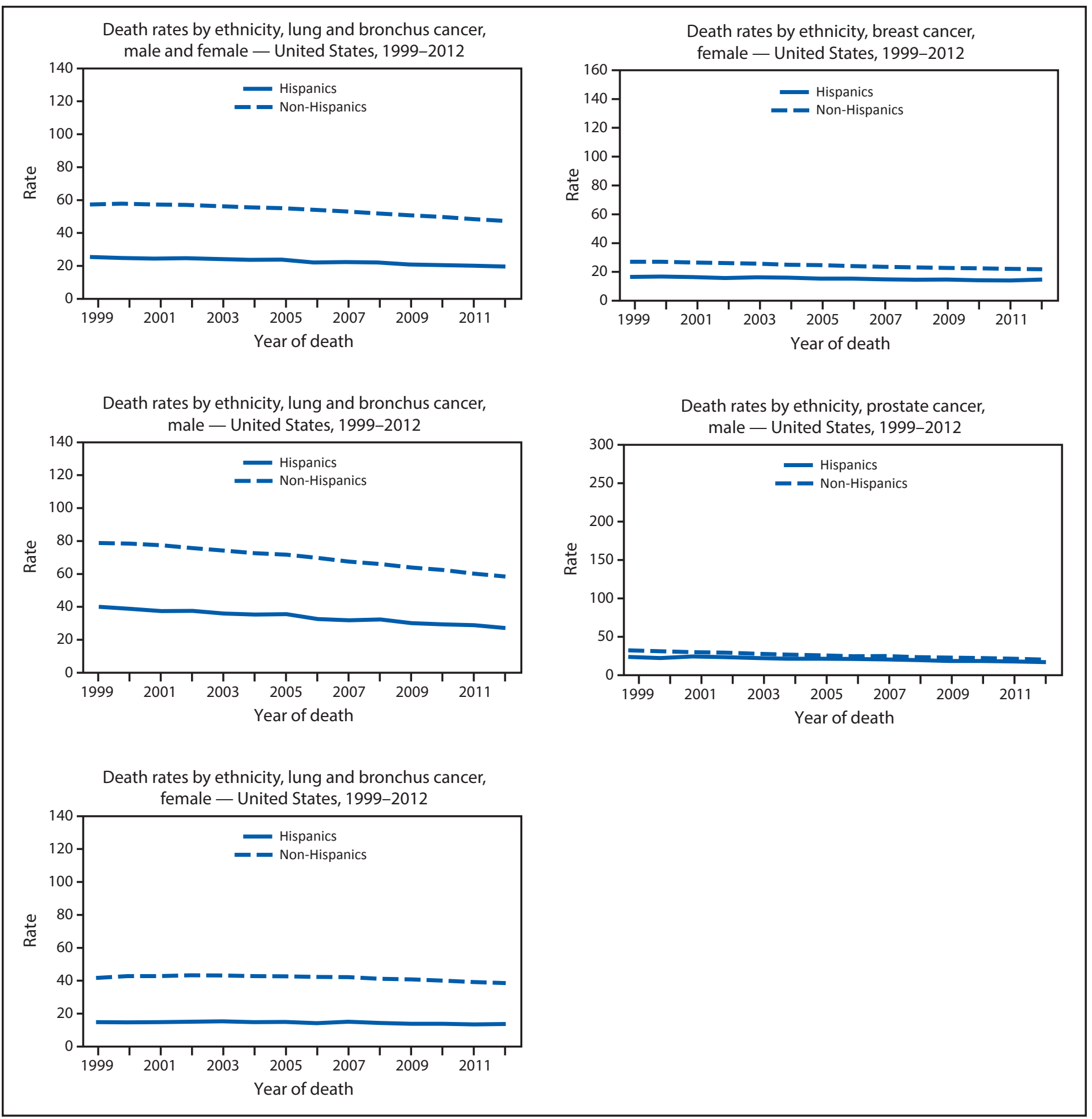

* Rates are the number of deaths per 100,000 persons and are age-adjusted to the 2000 U.S. standard population (19 age groups - Census P25-1130). For more information, see USCS technical notes (http://www.cdc.gov/cancer/npcr/uscs/pdf/uscs-2012-technical-notes.pdf).

† Data for specified ethnic populations should be interpreted with caution. For more information, see USCS technical notes (http://www.cdc.gov/cancer/npcr/uscs/ pdf/uscs-2012-technical-notes.pdf\#nameddest=IntRaceEthnicityData).

$\S$ Data are from the National Vital Statistics System (NVSS). Data for death rates cover $100 \%$ of the U.S. population. Caution should be used when comparing incidence and death rates because of potential differences in population coverage. 
TABLE 1. Reported number and age-adjusted rate* of invasive ${ }^{\dagger}$ cancer cases, by primary cancer site - United States, $2012^{\S}$

\begin{tabular}{|c|c|c|}
\hline \multirow[b]{2}{*}{ Cancer site } & \multicolumn{2}{|c|}{ All races/ethnicities } \\
\hline & No. & Rate \\
\hline All cancer sites combined & $1,529,078$ & 440.3 \\
\hline Oral cavity and pharynx & 39,879 & 11.2 \\
\hline Lip & 1,842 & 0.5 \\
\hline Tongue & 12,374 & 3.4 \\
\hline Salivary gland & 4,254 & 1.3 \\
\hline Floor of mouth & 1,936 & 0.5 \\
\hline Gum and other mouth & 5,331 & 1.5 \\
\hline Nasopharynx & 1,758 & 0.5 \\
\hline Tonsil & 7,330 & 2.0 \\
\hline Oropharynx & 1,733 & 0.5 \\
\hline Hypopharynx & 2,281 & 0.6 \\
\hline Other oral cavity and pharynx & 1,040 & 0.3 \\
\hline Digestive system & 273,535 & 78.3 \\
\hline Esophagus & 15,993 & 4.5 \\
\hline Stomach & 22,623 & 6.6 \\
\hline Small intestine & 7,894 & 2.3 \\
\hline Colon and rectum & 134,784 & 38.9 \\
\hline Colon excluding rectum & 95,962 & 27.8 \\
\hline Rectum and rectosigmoid junction & 38,822 & 11.1 \\
\hline Anus, anal canal, and anorectum & 6,338 & 1.8 \\
\hline Liver and Intrahepatic bile duct & 28,012 & 7.7 \\
\hline Gallbladder & 3,835 & 1.1 \\
\hline Other biliary & 5,963 & 1.7 \\
\hline Pancreas & 43,213 & 12.3 \\
\hline Retroperitoneum & 1,321 & 0.4 \\
\hline Peritoneum, omentum, and mesentery & 1,924 & 0.5 \\
\hline Other digestive organs & 1,635 & 0.5 \\
\hline Respiratory system & 225,933 & 64.7 \\
\hline Nose, nasal cavity, and middle ear & 2,274 & 0.7 \\
\hline Larynx & 12,152 & 3.4 \\
\hline Lung and bronchus & 210,828 & 60.4 \\
\hline Pleura & 105 & 0 \\
\hline $\begin{array}{l}\text { Trachea, mediastinum, and other } \\
\text { respiratory organs }\end{array}$ & 574 & 0.2 \\
\hline Bones and joints & 2,951 & 0.9 \\
\hline Soft tissue including heart & 10,728 & 3.2 \\
\hline Skin excluding basal and squamous & 73,181 & 21.5 \\
\hline Melanoma of the skin & 67,753 & 19.9 \\
\hline Other nonepithelial skin & 5,428 & 1.6 \\
\hline Male and female breast & 226,272 & 65.6 \\
\hline Female breast & 224,147 & 122.2 \\
\hline Male breast & 2,125 & 1.4 \\
\hline Female genital system & 90,303 & 48.8 \\
\hline Cervix & 12,042 & 7.4 \\
\hline Corpus and uterus, NOS & 49,154 & 25.7 \\
\hline Corpus & 47,570 & 24.9 \\
\hline Uterus, NOS & 1,584 & 0.8 \\
\hline Ovary & 20,785 & 11.3 \\
\hline Vagina & 1,296 & 0.7 \\
\hline Vulva & 4,851 & 2.6 \\
\hline Other female genital organs & 2,175 & 1.2 \\
\hline
\end{tabular}

TABLE 1. (Continued) Reported number and age-adjusted rate* of invasive ${ }^{\dagger}$ cancer cases, by primary cancer site - United States, $2012^{\S}$

\begin{tabular}{lrr}
\hline & \multicolumn{2}{c}{ All races/ethnicities } \\
\cline { 2 - 3 } Cancer site & \multicolumn{1}{c}{ No. } & \multicolumn{1}{c}{ Rate } \\
\hline Male genital system & 187,308 & 111.8 \\
Prostate & 177,489 & 105.3 \\
Testis & 8,189 & 5.5 \\
Penis & 1,283 & 0.8 \\
Other male genital organs & 347 & 0.2 \\
Urinary system & 128,103 & 37.0 \\
Urinary bladder & 69,974 & 20.2 \\
Kidney and renal pelvis & 55,231 & 15.9 \\
Ureter & 1,918 & 0.6 \\
Other urinary organs & 980 & 0.3 \\
Eye and orbit & 2,733 & 0.8 \\
Brain and other nervous system & 21,490 & 6.5 \\
Brain & 20,151 & 6.0 \\
Cranial nerves other nervous system & 1,339 & 0.4 \\
Endocrine system & 48,594 & 15.0 \\
Thyroid & 46,279 & 14.3 \\
Other endocrine including thymus & 2,315 & 0.7 \\
Lymphomas & 71,692 & 21.1 \\
Hodgkin lymphoma & 8,273 & 2.6 \\
Non-Hodgkin lymphoma & 63,419 & 18.5 \\
Myeloma & 21,829 & 6.3 \\
Leukemias & 44,396 & 13.2 \\
Acute lymphocytic leukemia & 4,846 & 1.6 \\
Chronic lymphocytic leukemia & 14,821 & 4.2 \\
Acute myeloid leukemia & 13,820 & 4.1 \\
Chronic myeloid leukemia & 5,543 & 1.7 \\
Other leukemias & 5,366 & 1.6 \\
Mesothelioma & 3,199 & 0.9 \\
Kaposi Sarcoma & 1,076 & 0.3 \\
Miscellaneous & 55,876 & 16.3 \\
\hline Abbra & & \\
\hline & & \\
& &
\end{tabular}

Abbreviation: NOS = not otherwise specified.

* Rates are the number of cases per 100,000 persons and are age-adjusted to the 2000 U.S. standard population (19 age groups - Census P25-1130). For more information, see USCS technical notes (http://www.cdc.gov/cancer/npcr/ uscs/pdf/uscs-2012-technical-notes.pdf).

+ Invasive cancer excludes basal and squamous cell carcinomas of the skin except when these occur on the skin of the genital organs, and in situ cancers except urinary bladder. Urinary bladder cancer includes invasive and in situ.

$\S$ Data are compiled from cancer registries that meet the data quality criteria for all invasive cancer sites combined (covering approximately $99 \%$ of the U.S. population). Registry-specific data quality information is available at http:// www.cdc.gov/cancer/npcr/uscs/pdf/uscs-2012-technical-notes.pdf. 
TABLE 2. Reported number and age-adjusted rate* of cancer deaths, by primary cancer site - United States, $2012^{\dagger}$

\begin{tabular}{|c|c|c|}
\hline \multirow[b]{2}{*}{ Cancer site } & \multicolumn{2}{|c|}{ All races/ethnicities } \\
\hline & No. & Rate \\
\hline All cancer sites combined & 582,607 & 166.4 \\
\hline Oral cavity and pharynx & 8,924 & 2.5 \\
\hline Lip & 71 & 0 \\
\hline Tongue & 2,224 & 0.6 \\
\hline Salivary gland & 869 & 0.2 \\
\hline Floor of mouth & 75 & 0 \\
\hline Gum and other mouth & 1,275 & 0.4 \\
\hline Nasopharynx & 666 & 0.2 \\
\hline Tonsil & 879 & 0.2 \\
\hline Oropharynx & 891 & 0.2 \\
\hline Hypopharynx & 356 & 0.1 \\
\hline Other oral cavity and pharynx & 1,618 & 0.4 \\
\hline Digestive system & 147,024 & 41.6 \\
\hline Esophagus & 14,649 & 4.1 \\
\hline Stomach & 11,191 & 3.2 \\
\hline Small intestine & 1,293 & 0.4 \\
\hline Colon and rectum & 51,516 & 14.7 \\
\hline Colon excluding rectum & 41,867 & 12.0 \\
\hline Rectum and rectosigmoid junction & 9,649 & 2.7 \\
\hline Anus, anal canal, and anorectum & 889 & 0.3 \\
\hline Liver and intrahepatic bile duct & 22,972 & 6.3 \\
\hline Gallbladder & 2,102 & 0.6 \\
\hline Other biliary & 1,519 & 0.4 \\
\hline Pancreas & 38,797 & 11.0 \\
\hline Retroperitoneum & 207 & 0.1 \\
\hline Peritoneum, omentum, and mesentery & 750 & 0.2 \\
\hline Other digestive organs & 1,139 & 0.3 \\
\hline Respiratory system & 161,851 & 46.2 \\
\hline Nose, nasal cavity, and middle ear & 458 & 0.1 \\
\hline Larynx & 3,662 & 1.0 \\
\hline Lung and bronchus & 157,423 & 45.0 \\
\hline Pleura & 82 & 0 \\
\hline $\begin{array}{l}\text { Trachea, mediastinum, and other } \\
\text { respiratory organs }\end{array}$ & 226 & 0.1 \\
\hline Bones and joints & 1,399 & 0.4 \\
\hline Soft tissue including heart & 4,559 & 1.3 \\
\hline Skin excluding basal and squamous & 12,463 & 3.6 \\
\hline Melanoma of the skin & 9,251 & 2.7 \\
\hline Other nonepithelial skin & 3,212 & 0.9 \\
\hline Male and female breast & 41,555 & 11.8 \\
\hline Female breast & 41,150 & 21.3 \\
\hline Male breast & 405 & 0.3 \\
\hline
\end{tabular}

TABLE 2. (Continued) Reported number and age-adjusted rate* of cancer deaths, by primary cancer site - United States, $2012^{\dagger}$

\begin{tabular}{lrr}
\hline & \multicolumn{2}{c}{ All races/ethnicities } \\
\cline { 2 - 3 } Cancer site & No. & Rate \\
\hline Female genital system & 29,405 & 15.2 \\
Cervix & 4,074 & 2.3 \\
Corpus and uterus, NOS & 8,911 & 4.5 \\
Corpus & 3,812 & 1.9 \\
Uterus, NOS & 5,099 & 2.6 \\
Ovary & 14,404 & 7.4 \\
Vagina & 429 & 0.2 \\
Vulva & 1,034 & 0.5 \\
Other female genital organs & 553 & 0.3 \\
Male genital system & 27,955 & 20.0 \\
Prostate & 27,244 & 19.6 \\
Testis & 386 & 0.3 \\
Penis & 273 & 0.2 \\
Other male genital organs & 52 & 0 \\
Urinary system & 29,594 & 8.5 \\
Urinary bladder & 15,245 & 4.4 \\
Kidney and renal pelvis & 13,518 & 3.8 \\
Ureter & 378 & 0.1 \\
Other urinary organs & 453 & 0.1 \\
Eye and orbit & 279 & 0.1 \\
Brain and other nervous system & 15,276 & 4.4 \\
Endocrine system & 2,660 & 0.8 \\
Thyroid & 1,690 & 0.5 \\
Other endocrine including thymus & 970 & 0.3 \\
Lymphomas & 21,518 & 6.2 \\
Hodgkin lymphoma & 1,130 & 0.3 \\
Non-Hodgkin lymphoma & 20,388 & 5.9 \\
Myeloma & 11,821 & 3.4 \\
Leukemias & 23,309 & 6.8 \\
Acute lymphocytic leukemia & 1,408 & 0.4 \\
Chronic lymphocytic leukemia & 4,598 & 1.3 \\
Acute myeloid leukemia & 9,484 & 2.8 \\
Chronic myeloid leukemia & 1,017 & 0.3 \\
Other leukemias & 6,802 & 2.0 \\
Mesothelioma & 0.8 \\
Miscellaneous & 11.5 \\
\hline Abbreviaton:NOS not & \\
\hline & & 0.3 \\
\hline
\end{tabular}

Abbreviation: $\mathrm{NOS}=$ not otherwise specified.

* Rates are the number of deaths per 100,000 persons and are age-adjusted to the 2000 U.S. standard population (19 age groups - Census P25-1130). For more information, see UCSC technical notes (http://www.cdc.gov/cancer/ $\mathrm{npcr/uscs/pdf/uscs-2012-technical-notes.pdf).}$

† Data are from the National Vital Statistics System (NVSS). 
TABLE 3. Reported number and age-adjusted rate* of invasive ${ }^{\dagger}$ cancer cases, all cancer sites combined, by geographic division and area - United States, $2012^{\S}$

\begin{tabular}{|c|c|c|}
\hline \multirow[b]{2}{*}{ Area } & \multicolumn{2}{|c|}{ All races/ethnicities } \\
\hline & No. & Rate \\
\hline Northeast & 311,842 & 474.3 \\
\hline New England & 81,778 & 469.6 \\
\hline Connecticut & 20,371 & 477.1 \\
\hline Maine & 8,417 & 475.1 \\
\hline Massachusetts & 35,774 & 463.1 \\
\hline New Hampshire & 7,576 & 473.5 \\
\hline Rhode Island & 6,095 & 484.0 \\
\hline Vermont & 3,545 & 449.0 \\
\hline Middle Atlantic & 230,064 & 476.1 \\
\hline New Jersey & 48,545 & 477.7 \\
\hline New York & 105,941 & 475.8 \\
\hline Pennsylvania & 75,578 & 476.1 \\
\hline Midwest & 345,611 & 448.8 \\
\hline East North Central & 237,817 & 446.2 \\
\hline Illinois & 64,402 & 459.0 \\
\hline Indiana & 31,852 & 439.4 \\
\hline Michigan & 51,809 & 444.5 \\
\hline Ohio & 59,848 & 438.0 \\
\hline Wisconsin & 29,906 & 447.7 \\
\hline West North Central & 107,794 & 454.7 \\
\hline lowa & 17,000 & 463.4 \\
\hline Kansas & 14,614 & 460.3 \\
\hline Minnesota & 27,833 & 462.8 \\
\hline Missouri & 31,643 & 450.6 \\
\hline Nebraska & 8,953 & 432.6 \\
\hline North Dakota & 3,577 & 450.5 \\
\hline South Dakota & 4,174 & 434.3 \\
\hline South & 566,112 & 434.9 \\
\hline South Atlantic & 307,360 & 430.4 \\
\hline Delaware & 5,306 & 483.5 \\
\hline District of Columbia & 2,954 & 474.9 \\
\hline Florida & 105,651 & 414.2 \\
\hline Georgia & 45,623 & 459.6 \\
\hline Maryland & 27,870 & 430.8 \\
\hline North Carolina & 48,367 & 443.0 \\
\hline South Carolina & 24,737 & 447.7 \\
\hline Virginia & 35,743 & 403.1 \\
\hline West Virginia & 11,109 & 461.3 \\
\hline
\end{tabular}

TABLE 3. (Continued) Reported number and age-adjusted rate* of invasive $^{\dagger}$ cancer cases, all cancer sites combined, by geographic division and area - United States, $2012^{\S}$

\begin{tabular}{lrr}
\hline & \multicolumn{2}{c}{ All races/ethnicities } \\
\cline { 2 - 3 } Area & \multicolumn{1}{c}{ No. } & Rate \\
\hline East South Central & 100,201 & 470.0 \\
Alabama & 25,225 & 451.0 \\
Kentucky & 25,845 & 515.1 \\
Mississippi & 15,084 & 457.4 \\
Tennessee & 34,047 & 459.4 \\
West South Central & 158,551 & 424.0 \\
Arkansas & 15,259 & 442.8 \\
Louisiana & 24,062 & 483.4 \\
Oklahoma & 18,785 & 442.8 \\
Texas & 100,445 & 406.2 \\
West & -9 & - \\
Mountain & - & - \\
Arizona & 27,680 & 373.4 \\
Colorado & 21,614 & 405.5 \\
Idaho & 7,452 & 437.0 \\
Montana & 5,424 & 434.4 \\
Nevada & - & - \\
New Mexico & 8,726 & 371.4 \\
Utah & 9,582 & 404.0 \\
Wyoming & 2,516 & 396.0 \\
Pacific & 222,519 & 418.1 \\
Alaska & 2,601 & 401.8 \\
California & 158,944 & 410.1 \\
Hawaii & 6,802 & 415.6 \\
Oregon & 19,531 & 422.2 \\
Washington & 34,641 & 460.1 \\
\hline
\end{tabular}

* Rates are the number of cases per 100,000 persons and are age-adjusted to the 2000 U.S. standard population (19 age groups - Census P25-1130). For more information, see USCS technical notes (http://www.cdc.gov/cancer/npcr/ uscs/pdf/uscs-2012-technical-notes.pdf).

† Invasive cancer excludes basal and squamous cell carcinomas of the skin except when these occur on the skin of the genital organs, and in situ cancers except urinary bladder. Urinary bladder cancer includes invasive and in situ.

$\S$ Data are compiled from cancer registries that meet the data quality criteria for all invasive cancer sites combined (covering approximately $99 \%$ of the U.S. population). Registry-specific data quality information is available at http:// www.cdc.gov/cancer/npcr/uscs/pdf/uscs-2012-technical-notes.pdf\#namedd est=RegistriesPubCriteria.

" Rates and counts are not presented for the West Census Region, the Mountain Census Division, or Nevada because data from Nevada are not included in this analysis. 
TABLE 4. Reported cancer deaths and age-adjusted death rates, ${ }^{*}$ all cancer sites combined, by geographic division and area - United States, $2012^{\dagger}$

\begin{tabular}{|c|c|c|}
\hline \multirow[b]{2}{*}{ Area } & \multicolumn{2}{|c|}{ All races/ethnicities } \\
\hline & No. & Rate \\
\hline United States & 582,607 & 166.4 \\
\hline Northeast & 110,175 & 164.1 \\
\hline New England & 28,904 & 162.6 \\
\hline Connecticut & 6,681 & 152.0 \\
\hline Maine & 3,226 & 179.0 \\
\hline Massachusetts & 12,864 & 163.3 \\
\hline New Hampshire & 2,660 & 167.7 \\
\hline Rhode Island & 2,148 & 163.6 \\
\hline Vermont & 1,325 & 164.8 \\
\hline Middle Atlantic & 81,271 & 164.7 \\
\hline New Jersey & 16,483 & 160.3 \\
\hline New York & 35,881 & 159.5 \\
\hline Pennsylvania & 28,907 & 174.8 \\
\hline Midwest & 135,511 & 174.1 \\
\hline East North Central & 94,939 & 176.9 \\
\hline Illinois & 24,562 & 175.5 \\
\hline Indiana & 13,368 & 184.2 \\
\hline Michigan & 20,496 & 174.3 \\
\hline Ohio & 25,261 & 182.1 \\
\hline Wisconsin & 11,252 & 166.1 \\
\hline West North Central & 40,572 & 167.8 \\
\hline lowa & 6,438 & 167.9 \\
\hline Kansas & 5,429 & 167.7 \\
\hline Minnesota & 9,424 & 155.6 \\
\hline Missouri & 12,919 & 182.0 \\
\hline Nebraska & 3,479 & 164.6 \\
\hline North Dakota & 1,253 & 150.7 \\
\hline South Dakota & 1,630 & 162.0 \\
\hline South & 221,435 & 171.4 \\
\hline South Atlantic & 118,858 & 165.8 \\
\hline Delaware & 1,935 & 176.1 \\
\hline District of Columbia & 1,081 & 178.4 \\
\hline Florida & 42,187 & 157.8 \\
\hline Georgia & 16,020 & 169.6 \\
\hline Maryland & 10,524 & 166.0 \\
\hline North Carolina & 18,405 & 170.5 \\
\hline South Carolina & 9,728 & 179.0 \\
\hline Virginia & 14,294 & 165.3 \\
\hline West Virginia & 4,684 & 191.1 \\
\hline
\end{tabular}

TABLE 4. (Continued) Reported cancer deaths and age-adjusted death rates, ${ }^{*}$ all cancer sites combined, by geographic division and area United States, $2012^{\dagger}$

\begin{tabular}{lrr}
\hline & \multicolumn{2}{c}{ All races/ethnicities } \\
\cline { 2 - 3 } Area & \multicolumn{1}{c}{ No. } & Rate \\
\hline East South Central & 40,547 & 192.0 \\
Alabama & 10,274 & 184.8 \\
Kentucky & 10,012 & 201.2 \\
Mississippi & 6,496 & 200.0 \\
Tennessee & 13,765 & 187.9 \\
West South Central & 62,030 & 170.5 \\
Arkansas & 6,540 & 188.6 \\
Louisiana & 9,308 & 190.5 \\
Oklahoma & 8,040 & 189.6 \\
Texas & 38,142 & 160.6 \\
West & 115,486 & 151.9 \\
Mountain & 34,819 & 147.7 \\
Arizona & 11,085 & 148.3 \\
Colorado & 7,306 & 143.7 \\
Idaho & 2,572 & 152.0 \\
Montana & 1,954 & 154.2 \\
Nevada & 4,610 & 163.8 \\
New Mexico & 3,461 & 148.0 \\
Utah & 2,876 & 128.7 \\
Wyoming & 955 & 154.6 \\
Pacific & $\mathbf{3 0}, 667$ & 153.7 \\
Alaska & 925 & 169.0 \\
California & 151.0 \\
Hawaii & 2,284 & 134.6 \\
Oregon & 7,832 & 168.3 \\
Washington & 11,951 & 161.8 \\
\hline
\end{tabular}

* Rates are the number of deaths per 100,000 persons and are age-adjusted to the 2000 U.S. standard population (19 age groups - Census P25-1130). For more information, see USCS technical notes (http://www.cdc.gov/cancer/npcr/ uscs/pdf/uscs-2012-technical-notes.pdf).

† Data are from the National Vital Statistics System (NVSS). 
TABLE 5. Reported number and rate* of invasive ${ }^{\dagger}$ cancer cases, by primary cancer site and age group — United States, 2012 ${ }^{\S}$

\begin{tabular}{|c|c|c|c|c|c|c|c|c|c|c|c|c|}
\hline \multirow[b]{3}{*}{ Cancer site } & \multicolumn{10}{|c|}{ Age group (yrs) } & & \\
\hline & \multicolumn{2}{|c|}{$<15$} & \multicolumn{2}{|c|}{$15-24$} & \multicolumn{2}{|c|}{ 25-39 } & \multicolumn{2}{|c|}{$40-64$} & \multicolumn{2}{|c|}{$\geq 65$} & \multicolumn{2}{|c|}{ Total } \\
\hline & No. & Rate & No. & Rate & No. & Rate & No. & Rate & No. & Rate & No. & Rate (crude) \\
\hline All cancer sites combined & 9,967 & 16.5 & 12,634 & 29 & 58,709 & 95.9 & 620,927 & 603 & 826,841 & $1,932.60$ & $1,529,078$ & 491.5 \\
\hline Oral cavity and pharynx & 84 & 0.1 & 259 & 0.6 & 1,152 & 1.9 & 21,140 & 20.5 & 17,244 & 40.3 & 39,879 & 12.8 \\
\hline Esophagus & -9 & - & - & - & 127 & 0.2 & 6,383 & 6.2 & 9,480 & 22.2 & 15,993 & 5.1 \\
\hline Stomach & - & - & - & - & 649 & 1.1 & 8,153 & 7.9 & 13,764 & 32.2 & 22,623 & 7.3 \\
\hline Colon and rectum & 34 & 0.1 & 340 & 0.8 & 3,526 & 5.8 & 51,746 & 50.2 & 79,138 & 185 & 134,784 & 43.3 \\
\hline Liver and intrahepatic bile duct & 222 & 0.4 & 78 & 0.2 & 292 & 0.5 & 14,698 & 14.3 & 12,722 & 29.7 & 28,012 & 9.0 \\
\hline Pancreas & - & - & - & - & 391 & 0.6 & 14,178 & 13.8 & 28,585 & 66.8 & 43,213 & 13.9 \\
\hline Larynx & - & - & - & - & 107 & 0.2 & 5,966 & 5.8 & 6,068 & 14.2 & 12,152 & 3.9 \\
\hline Lung and bronchus & 27 & 0 & 99 & 0.2 & 893 & 1.5 & 66,105 & 64.2 & 143,704 & 335.9 & 210,828 & 67.8 \\
\hline Melanomas of the skin & 107 & 0.2 & 897 & 2.1 & 5,507 & 9.0 & 28,901 & 28.1 & 32,341 & 75.6 & 67,753 & 21.8 \\
\hline Female breast & - & - & - & - & 9,605 & 31.5 & 114,962 & 218.7 & 99,396 & 411.9 & 224,147 & 141.8 \\
\hline Cervix & - & - & - & - & 2,795 & 9.2 & 6,730 & 12.8 & 2,368 & 9.8 & 12,042 & 7.6 \\
\hline Corpus and uterus, NOS & - & - & - & - & 1,616 & 5.3 & 26,341 & 50.1 & 21,129 & 87.6 & 49,154 & 31.1 \\
\hline Ovary & 102 & 0.3 & 348 & 1.6 & 1,071 & 3.5 & 9,564 & 18.2 & 9,700 & 40.2 & 20,785 & 13.2 \\
\hline Prostate & - & - & - & - & 77 & 0.3 & 77,236 & 153.2 & 100,156 & 536.9 & 177,489 & 115.9 \\
\hline Testis & 54 & 0.2 & 1,425 & 6.4 & 4,073 & 13.2 & 2,440 & 4.8 & 197 & 1.1 & 8,189 & 5.3 \\
\hline Urinary bladder ${ }^{\dagger \dagger}$ & 20 & 0 & 65 & 0.1 & 548 & 0.9 & 18,019 & 17.5 & 51,322 & 120.0 & 69,974 & 22.5 \\
\hline Kidney and renal pelvis & 527 & 0.9 & 180 & 0.4 & 1,986 & 3.2 & 25,557 & 24.8 & 26,981 & 63.1 & 55,231 & 17.8 \\
\hline Brain and nervous system & 2,103 & 3.5 & 968 & 2.2 & 2,084 & 3.4 & 8,127 & 7.9 & 8,208 & 19.2 & 21,490 & 6.9 \\
\hline Thyroid & 197 & 0.3 & 2,029 & 4.7 & 9,545 & 15.6 & 24,876 & 24.2 & 9,632 & 22.5 & 46,279 & 14.9 \\
\hline Hodgkin lymphoma & 340 & 0.6 & 1,599 & 3.7 & 2,245 & 3.7 & 2,614 & 2.5 & 1,475 & 3.4 & 8,273 & 2.7 \\
\hline Non-Hodgkin lymphoma & 595 & 1.0 & 933 & 2.1 & 2,899 & 4.7 & 23,101 & 22.4 & 35,891 & 83.9 & 63,419 & 20.4 \\
\hline Myeloma & - & - & - & - & 266 & 0.4 & 7,887 & 7.7 & 13,663 & 31.9 & 21,829 & 7.0 \\
\hline Leukemias & 2,948 & 4.9 & 1,163 & 2.7 & 2,243 & 3.7 & 13,517 & 13.1 & 24,525 & 57.3 & 44,396 & 14.3 \\
\hline Mesothelioma & - & - & - & - & 36 & 0.1 & 666 & 0.6 & 2,486 & 5.8 & 3,199 & 1.0 \\
\hline Kaposi Sarcoma & - & - & - & - & 321 & 0.5 & 448 & 0.4 & 269 & 0.6 & 1,076 & 0.3 \\
\hline
\end{tabular}

Abbreviation: $\mathrm{NOS}=$ not otherwise specified.

* Rates are the number of cases per 100,000 persons. For more information, see USCS technical notes (http://www.cdc.gov/cancer/npcr/uscs/pdf/uscs-2012-technicalnotes.pdf).

† Invasive cancer excludes basal and squamous cell carcinomas of the skin except when these occur on the skin of the genital organs, and in situ cancers except urinary bladder. Urinary bladder cancer includes invasive and in situ.

$\S$ Data are compiled from cancer registries that meet the data quality criteria for all invasive cancer sites combined (covering approximately $99 \%$ of the U.S. population). Registry-specific data quality information is available at http://www.cdc.gov/cancer/npcr/uscs/pdf/uscs-2012-technical-notes.pdf\#nameddest=RegistriesPubCriteria.

" Counts and rates are suppressed if $<16$ cases were reported in a specific category. Some counts and rates are suppressed as complementary cell suppression. 
TABLE 6. Reported number and rate* of cancer deaths, by primary cancer site and age group — United States, $2012^{\dagger}$

\begin{tabular}{|c|c|c|c|c|c|c|c|c|c|c|c|c|}
\hline \multirow[b]{3}{*}{ Cancer site } & \multicolumn{10}{|c|}{ Age group (yrs) } & & \\
\hline & \multicolumn{2}{|c|}{$<15$} & \multicolumn{2}{|c|}{$15-24$} & \multicolumn{2}{|c|}{$25-39$} & \multicolumn{2}{|c|}{$40-64$} & \multicolumn{2}{|c|}{$\geq 65$} & \multicolumn{2}{|c|}{ Total (all ages) } \\
\hline & No. & Rate & No. & Rate & No. & Rate & No. & Rate & No. & Rate & No. & Rate (crude) \\
\hline All cancer sites combined & 1,367 & 2.2 & 1,574 & 3.6 & 7,391 & 12 & 168,778 & 162.5 & 403,497 & 935.2 & 582,607 & 185.6 \\
\hline Oral cavity and pharynx & — & - & - & - & 128 & 0.2 & 3,677 & 3.5 & 5,100 & 11.8 & 8,924 & 2.8 \\
\hline Esophagus & - & - & - & - & 74 & 0.1 & 5,358 & 5.2 & 9,213 & 21.4 & 14,649 & 4.7 \\
\hline Stomach & - & - & - & - & 271 & 0.4 & 3,590 & 3.5 & 7,309 & 16.9 & 11,191 & 3.6 \\
\hline Colon and rectum & - & - & - & - & 775 & 1.3 & 15,199 & 14.6 & 35,480 & 82.2 & 51,516 & 16.4 \\
\hline Liver and intrahepatic bile duct & 32 & 0.1 & 34 & 0.1 & 186 & 0.3 & 9,837 & 9.5 & 12,883 & 29.9 & 22,927 & 7.3 \\
\hline Pancreas & - & - & - & - & 151 & 0.2 & 10,962 & 10.6 & 27,676 & 64.1 & 38,797 & 12.4 \\
\hline Larynx & - & - & - & - & 18 & 0 & 1,374 & 1.3 & 2,269 & 5.3 & 3,662 & 1.2 \\
\hline Lung and bronchus & - & - & - & - & 430 & 0.7 & 43,463 & 41.8 & 113,499 & 263.1 & 157,423 & 50.2 \\
\hline Melanomas of the skin & - & - & - & - & 300 & 0.5 & 3,179 & 3.1 & 5,746 & 13.3 & 9,251 & 2.9 \\
\hline Female breast & - & - & - & - & 971 & 3.2 & 16,104 & 30.4 & 24,062 & 98.9 & 41,150 & 25.8 \\
\hline Cervix & - & - & - & - & 437 & 1.4 & 2,259 & 4.3 & 1,369 & 5.6 & 4,074 & 2.6 \\
\hline Corpus and uterus, NOS & - & - & - & - & 108 & 0.4 & 2,855 & 5.4 & 5,944 & 24.4 & 8,911 & 5.6 \\
\hline Ovary & - & - & - & - & 176 & 0.6 & 4,822 & 9.1 & 9,378 & 38.6 & 14,404 & 9.0 \\
\hline Prostate & - & - & - & - & - & - & 2,962 & 5.8 & 24,271 & 128.9 & 27,244 & 17.6 \\
\hline Testis & - & - & - & - & 131 & 0.4 & 149 & 0.3 & 51 & 0.3 & 386 & 0.2 \\
\hline Urinary bladder & - & - & - & - & 35 & 0.1 & 2,390 & 2.3 & 12,814 & 29.7 & 15,245 & 4.9 \\
\hline Kidney and renal pelvis & 30 & 0 & 31 & 0.1 & 109 & 0.2 & 4,134 & 4.0 & 9,214 & 21.4 & 13,518 & 4.3 \\
\hline Brain and nervous system & 458 & 0.7 & 252 & 0.6 & 744 & 1.2 & 6,177 & 5.9 & 7,645 & 17.7 & 15,276 & 4.9 \\
\hline Thyroid & - & - & - & - & 23 & 0 & 450 & 0.4 & 1,212 & 2.8 & 1,690 & 0.5 \\
\hline Hodgkin lymphoma & - & - & - & - & 134 & 0.2 & 353 & 0.3 & 604 & 1.4 & 1,130 & 0.4 \\
\hline Non-Hodgkin lymphoma & 36 & 0.1 & 85 & 0.2 & 344 & 0.6 & 4,304 & 4.1 & 15,619 & 36.2 & 20,388 & 6.5 \\
\hline Myeloma & - & - & - & - & 32 & 0.1 & 2,624 & 2.5 & 9,164 & 21.2 & 11,821 & 3.8 \\
\hline Leukemias & 378 & 0.6 & 356 & 0.8 & 649 & 1.1 & 4,627 & 4.5 & 17,299 & 40.1 & 23,309 & 7.4 \\
\hline Mesothelioma & - & - & - & - & 16 & 0 & 479 & 0.5 & 2,190 & 5.1 & 2,686 & 0.9 \\
\hline
\end{tabular}

Abbreviation: NOS = not otherwise specified.

* Rates are the number of deaths per 100,000 persons. For more information, see USCS technical notes (http://www.cdc.gov/cancer/npcr/uscs/pdf/uscs-2012-technicalnotes.pdf).

† Data are from the National Vital Statistics System (NVSS).

$\S$ Counts and rates are suppressed if $<16$ deaths were reported in a specific category. Some counts and rates are suppressed as complementary cell suppression. 
TABLE 7. Reported number and age-adjusted rate* of invasive ${ }^{\dagger}$ cancer cases, by primary cancer site and sex - United States, $2012^{\S}$

\begin{tabular}{|c|c|c|c|c|c|c|}
\hline \multirow[b]{2}{*}{ Cancer site } & \multicolumn{2}{|c|}{ Male } & \multicolumn{2}{|c|}{ Female } & \multicolumn{2}{|c|}{ Total } \\
\hline & No. & Rate & No. & Rate & No. & Rate \\
\hline All cancer sites combined & 767,366 & 483.0 & 761,712 & 411.7 & $1,529,078$ & 440.3 \\
\hline Oral cavity and pharynx & 27,997 & 16.8 & 11,882 & 6.3 & 39,879 & 11.2 \\
\hline Lip & 1,353 & 0.9 & 489 & 0.3 & 1,842 & 0.5 \\
\hline Tongue & 8,777 & 5.2 & 3,597 & 1.9 & 12,374 & 3.4 \\
\hline Salivary gland & 2,404 & 1.6 & 1,850 & 1.0 & 4,254 & 1.3 \\
\hline Floor of mouth & 1,333 & 0.8 & 603 & 0.3 & 1,936 & 0.5 \\
\hline Gum and other mouth & 2,915 & 1.8 & 2,416 & 1.3 & 5,331 & 1.5 \\
\hline Nasopharynx & 1,263 & 0.8 & 495 & 0.3 & 1,758 & 0.5 \\
\hline Tonsil & 5,977 & 3.4 & 1,353 & 0.7 & 7,330 & 2.0 \\
\hline Oropharynx & 1,325 & 0.8 & 408 & 0.2 & 1,733 & 0.5 \\
\hline Hypopharynx & 1,838 & 1.1 & 443 & 0.2 & 2,281 & 0.6 \\
\hline Other oral cavity and pharynx & 812 & 0.5 & 228 & 0.1 & 1,040 & 0.3 \\
\hline Digestive system & 151,713 & 95.6 & 121,822 & 63.9 & 273,535 & 78.3 \\
\hline Esophagus & 12,628 & 7.8 & 3,365 & 1.7 & 15,993 & 4.5 \\
\hline Stomach & 13,964 & 9.0 & 8,659 & 4.6 & 22,623 & 6.6 \\
\hline Small intestine & 4,187 & 2.6 & 3,707 & 2.0 & 7,894 & 2.3 \\
\hline Colon and rectum & 70,204 & 44.8 & 64,580 & 34.1 & 134,784 & 38.9 \\
\hline Colon excluding rectum & 47,448 & 30.7 & 48,514 & 25.4 & 95,962 & 27.8 \\
\hline Rectum and rectosigmoid junction & 22,756 & 14.1 & 16,066 & 8.6 & 38,822 & 11.1 \\
\hline Anus, anal canal, and anorectum & 2,304 & 1.4 & 4,034 & 2.1 & 6,338 & 1.8 \\
\hline Liver and Intrahepatic bile duct & 20,207 & 11.8 & 7,805 & 4.0 & 28,012 & 7.7 \\
\hline Gallbladder & 1,221 & 0.8 & 2,614 & 1.4 & 3,835 & 1.1 \\
\hline Other biliary & 3,246 & 2.1 & 2,717 & 1.4 & 5,963 & 1.7 \\
\hline Pancreas & 22,101 & 14.1 & 21,112 & 10.9 & 43,213 & 12.3 \\
\hline Retroperitoneum & 653 & 0.4 & 668 & 0.4 & 1,321 & 0.4 \\
\hline Peritoneum, omentum, and mesentery & 157 & 0.1 & 1,767 & 0.9 & 1,924 & 0.5 \\
\hline Other digestive organs & 841 & 0.5 & 794 & 0.4 & 1,635 & 0.5 \\
\hline Respiratory system & 122,785 & 78.5 & 103,148 & 54.0 & 225,933 & 64.7 \\
\hline Nose, nasal cavity, and middle ear & 1,376 & 0.9 & 898 & 0.5 & 2,274 & 0.7 \\
\hline Larynx & 9,565 & 5.8 & 2,587 & 1.3 & 12,152 & 3.4 \\
\hline Lung and bronchus & 111,395 & 71.6 & 99,433 & 52.1 & 210,828 & 60.4 \\
\hline Pleura & 60 & 0 & 45 & 0 & 105 & 0 \\
\hline Trachea, mediastinum, and other respiratory organs & 389 & 0.3 & 185 & 0.1 & 574 & 0.2 \\
\hline Bones and joints & 1,674 & 1.1 & 1,277 & 0.8 & 2,951 & 0.9 \\
\hline Soft tissue including heart & 5,881 & 3.8 & 4,847 & 2.8 & 10,728 & 3.2 \\
\hline Skin excluding basal and squamous & 42,992 & 27.8 & 30,189 & 17.0 & 73,181 & 21.5 \\
\hline Melanoma of the skin & 39,673 & 25.5 & 28,080 & 15.9 & 67,753 & 19.9 \\
\hline Other nonepithelial skin & 3,319 & 2.3 & 2,109 & 1.1 & 5,428 & 1.6 \\
\hline Male and female breast & NA & NA & NA & NA & 226,272 & 65.6 \\
\hline Female breast & NA & NA & 224,147 & 122.2 & NA & NA \\
\hline Male breast & 2,125 & 1.4 & NA & NA & NA & NA \\
\hline Female genital system & NA & NA & 90,303 & 48.8 & NA & NA \\
\hline Cervix & NA & NA & 12,042 & 7.4 & NA & NA \\
\hline Corpus and uterus, NOS & NA & NA & 49,154 & 25.7 & NA & NA \\
\hline Corpus & NA & NA & 47,570 & 24.9 & NA & NA \\
\hline Uterus, NOS & NA & NA & 1,584 & 0.8 & NA & NA \\
\hline Ovary & NA & NA & 20,785 & 11.3 & NA & NA \\
\hline Vagina & NA & NA & 1,296 & 0.7 & NA & NA \\
\hline Vulva & NA & NA & 4,851 & 2.6 & NA & NA \\
\hline Other female genital organs & NA & NA & 2,175 & 1.2 & NA & NA \\
\hline
\end{tabular}

See table footnotes on page 40 . 
TABLE 7. (Continued) Reported number and age-adjusted rate* of invasive ${ }^{\dagger}$ cancer cases, by primary cancer site and sex - United States, $2012^{\S}$

\begin{tabular}{|c|c|c|c|c|c|c|}
\hline \multirow[b]{2}{*}{ Cancer site } & \multicolumn{2}{|c|}{ Male } & \multicolumn{2}{|c|}{ Female } & \multicolumn{2}{|c|}{ Total } \\
\hline & No. & Rate & No. & Rate & No. & Rate \\
\hline Male genital system & 187,308 & 111.8 & NA & NA & NA & NA \\
\hline Prostate & 177,489 & 105.3 & NA & NA & NA & NA \\
\hline Testis & 8,189 & 5.5 & NA & NA & NA & NA \\
\hline Penis & 1,283 & 0.8 & NA & NA & NA & NA \\
\hline Other male genital organs & 347 & 0.2 & NA & NA & NA & NA \\
\hline Urinary system & 89,322 & 58.0 & 38,781 & 20.5 & 128,103 & 37.0 \\
\hline Urinary bladder & 53,006 & 35.4 & 16,968 & 8.7 & 69,974 & 20.2 \\
\hline Kidney and renal pelvis & 34,459 & 21.3 & 20,772 & 11.2 & 55,231 & 15.9 \\
\hline Ureter & 1,195 & 0.8 & 723 & 0.4 & 1,918 & 0.6 \\
\hline Other urinary organs & 662 & 0.5 & 318 & 0.2 & 980 & 0.3 \\
\hline Eye and orbit & 1,494 & 1.0 & 1,239 & 0.7 & 2,733 & 0.8 \\
\hline Brain and other nervous system & 11,951 & 7.6 & 9,539 & 5.5 & 21,490 & 6.5 \\
\hline Brain & 11,263 & 7.2 & 8,888 & 5.1 & 20,151 & 6.0 \\
\hline Cranial nerves other nervous system & 688 & 0.5 & 651 & 0.4 & 1,339 & 0.4 \\
\hline Endocrine system & 12,497 & 7.9 & 36,097 & 21.9 & 48,594 & 15.0 \\
\hline Thyroid & 11,313 & 7.1 & 34,966 & 21.3 & 46,279 & 14.3 \\
\hline Other endocrine including thymus & 1,184 & 0.8 & 1,131 & 0.7 & 2,315 & 0.7 \\
\hline Lymphomas & 39,074 & 25.3 & 32,618 & 17.8 & 71,692 & 21.1 \\
\hline Hodgkin lymphoma & 4,569 & 3.0 & 3,704 & 2.3 & 8,273 & 2.6 \\
\hline Non-Hodgkin lymphoma & 34,505 & 22.3 & 28,914 & 15.5 & 63,419 & 18.5 \\
\hline Myeloma & 12,199 & 7.8 & 9,630 & 5.0 & 21,829 & 6.3 \\
\hline Leukemias & 25,699 & 16.9 & 18,697 & 10.3 & 44,396 & 13.2 \\
\hline Acute lymphocytic leukemia & 2,732 & 1.8 & 2,114 & 1.4 & 4,846 & 1.6 \\
\hline Chronic lymphocytic leukemia & 9,004 & 5.8 & 5,817 & 3.0 & 14,821 & 4.2 \\
\hline Acute myeloid leukemia & 7,646 & 5.1 & 6,174 & 3.4 & 13,820 & 4.1 \\
\hline Chronic myeloid leukemia & 3,118 & 2.1 & 2,425 & 1.4 & 5,543 & 1.7 \\
\hline Other leukemias & 3,199 & 2.1 & 2,167 & 1.2 & 5,366 & 1.6 \\
\hline Mesothelioma & 2,407 & 1.7 & 792 & 0.4 & 3,199 & 0.9 \\
\hline Kaposi Sarcoma & 951 & 0.6 & 125 & 0.1 & 1,076 & 0.3 \\
\hline Miscellaneous & 29,297 & 19.6 & 26,579 & 13.7 & 55,876 & 16.3 \\
\hline
\end{tabular}

Abbreviations: $\mathrm{NA}=$ not applicable; $\mathrm{NOS}=$ not otherwise specified.

* Rates are the number of cases per 100,000 persons and are age-adjusted to the 2000 U.S. standard population (19 age groups - Census P25-1130). For more information, see USCS technical notes (http://www.cdc.gov/cancer/npcr/uscs/pdf/uscs-2012-technical-notes.pdf).

${ }^{\dagger}$ Invasive cancer excludes basal and squamous cell carcinomas of the skin except when these occur on the skin of the genital organs, and in situ cancers except urinary bladder. Urinary bladder cancer includes invasive and in situ.

$\S$ Data are compiled from cancer registries that meet the data quality criteria for all invasive cancer sites combined (covering approximately $99 \%$ of the U.S. population). Registry-specific data quality information is available at http://www.cdc.gov/cancer/npcr/uscs/pdf/uscs-2012-technical-notes.pdf\#nameddest=RegistriesPubCriteria. 
TABLE 8. Reported number and age-adjusted rate* of cancer deaths, by primary cancer site and sex - United States, $2012^{\dagger}$

\begin{tabular}{|c|c|c|c|c|c|c|}
\hline \multirow[b]{2}{*}{ Cancer site } & \multicolumn{2}{|c|}{ Male } & \multicolumn{2}{|c|}{ Female } & \multicolumn{2}{|c|}{ Total } \\
\hline & No. & Rate & No. & Rate & No. & Rate \\
\hline All cancer sites combined & 305,661 & 200.6 & 276,946 & 141.9 & 582,607 & 166.4 \\
\hline Oral cavity and pharynx & 6,263 & 3.9 & 2,661 & 1.3 & 8,924 & 2.5 \\
\hline Lip & 44 & 0 & 27 & 0 & 71 & 0 \\
\hline Tongue & 1,488 & 0.9 & 736 & 0.4 & 2,224 & 0.6 \\
\hline Salivary gland & 581 & 0.4 & 288 & 0.1 & 869 & 0.2 \\
\hline Floor of mouth & 54 & 0 & 21 & 0 & 75 & 0 \\
\hline Gum and other mouth & 746 & 0.5 & 529 & 0.3 & 1,275 & 0.4 \\
\hline Nasopharynx & 454 & 0.3 & 212 & 0.1 & 666 & 0.2 \\
\hline Tonsil & 714 & 0.4 & 165 & 0.1 & 879 & 0.2 \\
\hline Oropharynx & 653 & 0.4 & 238 & 0.1 & 891 & 0.2 \\
\hline Hypopharynx & 268 & 0.2 & 88 & 0 & 356 & 0.1 \\
\hline Other oral cavity and pharynx & 1,261 & 0.8 & 357 & 0.2 & 1,618 & 0.4 \\
\hline Digestive system & 83,782 & 53.4 & 63,242 & 32.0 & 147,024 & 41.6 \\
\hline Esophagus & 11,697 & 7.3 & 2,952 & 1.5 & 14,649 & 4.1 \\
\hline Stomach & 6,611 & 4.3 & 4,580 & 2.4 & 11,191 & 3.2 \\
\hline Small intestine & 689 & 0.5 & 604 & 0.3 & 1,293 & 0.4 \\
\hline Colon and rectum & 26,866 & 17.6 & 24,650 & 12.4 & 51,516 & 14.7 \\
\hline Colon excluding rectum & 21,383 & 14.1 & 20,484 & 10.3 & 41,867 & 12.0 \\
\hline Rectum and rectosigmoid junction & 5,483 & 3.5 & 4,166 & 2.1 & 9,649 & 2.7 \\
\hline Anus, anal canal, and anorectum & 368 & 0.2 & 521 & 0.3 & 889 & 0.3 \\
\hline Liver and intrahepatic bile duct & 15,563 & 9.4 & 7,409 & 3.8 & 22,972 & 6.3 \\
\hline Gallbladder & 687 & 0.5 & 1,415 & 0.7 & 2,102 & 0.6 \\
\hline Other biliary & 739 & 0.5 & 780 & 0.4 & 1,519 & 0.4 \\
\hline Pancreas & 19,718 & 12.7 & 19,079 & 9.6 & 38,797 & 11.0 \\
\hline Retroperitoneum & 110 & 0.1 & 97 & 0.1 & 207 & 0.1 \\
\hline Peritoneum, omentum, and mesentery & 78 & 0 & 672 & 0.3 & 750 & 0.2 \\
\hline Other digestive organs & 656 & 0.4 & 483 & 0.2 & 1,139 & 0.3 \\
\hline Respiratory system & 90,091 & 58.4 & 71,760 & 37.0 & 161,851 & 46.2 \\
\hline Nose, nasal cavity, and middle ear & 285 & 0.2 & 173 & 0.1 & 458 & 0.1 \\
\hline Larynx & 2,925 & 1.8 & 737 & 0.4 & 3,662 & 1.0 \\
\hline Lung and bronchus & 86,689 & 56.2 & 70,734 & 36.4 & 157,423 & 45.0 \\
\hline Pleura & 51 & 0 & 31 & 0 & 82 & 0 \\
\hline Trachea, mediastinum, and other respiratory organs & 141 & 0.1 & 85 & 0 & 226 & 0.1 \\
\hline Bones and joints & 818 & 0.5 & 581 & 0.3 & 1,399 & 0.4 \\
\hline Soft tissue including heart & 2,394 & 1.6 & 2,165 & 1.2 & 4,559 & 1.3 \\
\hline Skin excluding basal and squamous & 8,329 & 5.5 & 4,134 & 2.1 & 12,463 & 3.6 \\
\hline Melanoma of the skin & 6,013 & 4.0 & 3,238 & 1.7 & 9,251 & 2.7 \\
\hline Other nonepithelial skin & 2,316 & 1.6 & 896 & 0.4 & 3,212 & 0.9 \\
\hline Male and female breast & NA & NA & NA & NA & 41,555 & 11.8 \\
\hline Female breast & NA & NA & 41,150 & 21.3 & NA & NA \\
\hline Male breast & 405 & 0.3 & NA & NA & NA & NA \\
\hline
\end{tabular}

See table footnotes on page 42 . 
TABLE 8. (Continued) Reported number and age-adjusted rate* of cancer deaths, by primary cancer site and sex — United States, $2012^{\dagger}$

\begin{tabular}{|c|c|c|c|c|c|c|}
\hline \multirow[b]{2}{*}{ Cancer site } & \multicolumn{2}{|c|}{ Male } & \multicolumn{2}{|c|}{ Female } & \multicolumn{2}{|c|}{ Total } \\
\hline & No. & Rate & No. & Rate & No. & Rate \\
\hline Female genital system & NA & NA & 29,405 & 15.2 & NA & NA \\
\hline Cervix & NA & NA & 4,074 & 2.3 & NA & NA \\
\hline Corpus and uterus, NOS & NA & NA & 8,911 & 4.5 & NA & NA \\
\hline Corpus & NA & NA & 3,812 & 1.9 & NA & NA \\
\hline Uterus, NOS & NA & NA & 5,099 & 2.6 & NA & NA \\
\hline Ovary & NA & NA & 14,404 & 7.4 & NA & NA \\
\hline Vagina & NA & NA & 429 & 0.2 & NA & NA \\
\hline Vulva & NA & NA & 1,034 & 0.5 & NA & NA \\
\hline Other female genital organs & NA & NA & 553 & 0.3 & NA & NA \\
\hline Male genital system & 27,955 & 20.0 & NA & NA & NA & NA \\
\hline Prostate & 27,244 & 19.6 & NA & NA & NA & NA \\
\hline Testis & 386 & 0.3 & NA & NA & NA & NA \\
\hline Penis & 273 & 0.2 & NA & NA & NA & NA \\
\hline Other male genital organs & 52 & 0 & NA & NA & NA & NA \\
\hline Urinary system & 20,136 & 13.6 & 9,458 & 4.7 & 29,594 & 8.5 \\
\hline Urinary bladder & 10,886 & 7.6 & 4,359 & 2.1 & 15,245 & 4.4 \\
\hline Kidney and renal pelvis & 8,772 & 5.6 & 4,746 & 2.4 & 13,518 & 3.8 \\
\hline Ureter & 212 & 0.1 & 166 & 0.1 & 378 & 0.1 \\
\hline Other urinary organs & 266 & 0.2 & 187 & 0.1 & 453 & 0.1 \\
\hline Eye and orbit & 164 & 0.1 & 115 & 0.1 & 279 & 0.1 \\
\hline Brain and other nervous system & 8,666 & 5.4 & 6,610 & 3.5 & 15,276 & 4.4 \\
\hline Endocrine system & 1,264 & 0.8 & 1,396 & 0.7 & 2,660 & 0.8 \\
\hline Thyroid & 775 & 0.5 & 915 & 0.5 & 1,690 & 0.5 \\
\hline Other endocrine including thymus & 489 & 0.3 & 481 & 0.3 & 970 & 0.3 \\
\hline Lymphomas & 11,902 & 8.0 & 9,616 & 4.9 & 21,518 & 6.2 \\
\hline Hodgkin lymphoma & 638 & 0.4 & 492 & 0.3 & 1,130 & 0.3 \\
\hline Non-Hodgkin lymphoma & 11,264 & 7.6 & 9,124 & 4.6 & 20,388 & 5.9 \\
\hline Myeloma & 6,338 & 4.2 & 5,483 & 2.8 & 11,821 & 3.4 \\
\hline Leukemias & 13,466 & 9.2 & 9,843 & 5.1 & 23,309 & 6.8 \\
\hline Acute lymphocytic leukemia & 810 & 0.5 & 598 & 0.3 & 1,408 & 0.4 \\
\hline Chronic lymphocytic leukemia & 2,837 & 2.0 & 1,761 & 0.9 & 4,598 & 1.3 \\
\hline Acute myeloid leukemia & 5,401 & 3.6 & 4,083 & 2.2 & 9,484 & 2.8 \\
\hline Chronic myeloid leukemia & 546 & 0.4 & 471 & 0.2 & 1,017 & 0.3 \\
\hline Other leukemias & 3,872 & 2.7 & 2,930 & 1.5 & 6,802 & 2.0 \\
\hline Mesothelioma & 2,112 & 1.5 & 574 & 0.3 & 2,686 & 0.8 \\
\hline Miscellaneous & 21,538 & 14.1 & 18,738 & 9.4 & 40,276 & 11.5 \\
\hline
\end{tabular}

Abbreviations: NA = not applicable; NOS = not otherwise specified.

* Rates are the number of deaths per 100,000 persons and are age-adjusted to the 2000 U.S. standard population (19 age groups - Census P25-1130). For more information, see USCS technical notes (http://www.cdc.gov/cancer/npcr/uscs/pdf/uscs-2012-technical-notes.pdf).

${ }^{\dagger}$ Data are from the National Vital Statistics System (NVSS). 
TABLE 9. Reported number and age-adjusted rate* of invasive ${ }^{\dagger}$ cancer cases, by primary cancer site and race ${ }^{\S}$-United States, $2012^{\Uparrow}$

\begin{tabular}{|c|c|c|c|c|c|c|c|c|c|c|}
\hline \multirow[b]{2}{*}{ Cancer site } & \multicolumn{2}{|c|}{$A \mathrm{Al} / \mathrm{AN}^{\S}$} & \multicolumn{2}{|c|}{$\left.A P\right|^{\S}$} & \multicolumn{2}{|c|}{ Black } & \multicolumn{2}{|c|}{ White } & \multicolumn{2}{|c|}{ Total } \\
\hline & No. & Rate & No. & Rate & No. & Rate & No. & Rate & No. & Rate \\
\hline All cancer sites combined & 8,139 & 269.0 & 45,364 & 285.7 & 165,559 & 446.1 & $1,282,703$ & 440.4 & $1,529,078$ & 440.3 \\
\hline Oral cavity and pharynx & 227 & 6.8 & 1,267 & 7.7 & 3,574 & 9.1 & 34,263 & 11.5 & 39,879 & 11.2 \\
\hline Lip & - ${ }^{* *}$ & - & - & - & 42 & 0.1 & 1,718 & 0.6 & 1,842 & 0.5 \\
\hline Tongue & 51 & 1.5 & 288 & 1.8 & 786 & 2.0 & 11,082 & 3.7 & 12,374 & 3.4 \\
\hline Salivary gland & 18 & 0.5 & 180 & 1.1 & 393 & 1.0 & 3,597 & 1.3 & 4,254 & 1.3 \\
\hline Floor of mouth & - & - & 26 & 0.2 & 206 & 0.5 & 1,666 & 0.5 & 1,936 & 0.5 \\
\hline Gum and other mouth & 24 & 0.8 & 207 & 1.3 & 511 & 1.4 & 4,500 & 1.5 & 5,331 & 1.5 \\
\hline Nasopharynx & 21 & 0.6 & 384 & 2.2 & 263 & 0.7 & 1,064 & 0.4 & 1,758 & 0.5 \\
\hline Tonsil & 45 & 1.2 & 81 & 0.5 & 625 & 1.5 & 6,512 & 2.2 & 7,330 & 2.0 \\
\hline Oropharynx & - & - & 20 & 0.1 & 241 & 0.6 & 1,449 & 0.5 & 1,733 & 0.5 \\
\hline Hypopharynx & 21 & 0.7 & 54 & 0.3 & 390 & 1.0 & 1,796 & 0.6 & 2,281 & 0.6 \\
\hline Other oral cavity and pharynx & 16 & 0.5 & - & - & 117 & 0.3 & 879 & 0.3 & 1,040 & 0.3 \\
\hline Digestive system & 1,722 & 58.4 & 11,016 & 72.1 & 34,404 & 94.8 & 223,154 & 75.7 & 273,535 & 78.3 \\
\hline Esophagus & 73 & 2.6 & 303 & 2.0 & 1,514 & 4.1 & 13,963 & 4.7 & 15,993 & 4.5 \\
\hline Stomach & 167 & 6.1 & 1,571 & 10.5 & 3,532 & 10.2 & 17,056 & 5.8 & 22,623 & 6.6 \\
\hline Small intestine & 25 & 0.8 & 181 & 1.1 & 1,301 & 3.5 & 6,300 & 2.2 & 7,894 & 2.3 \\
\hline Colon and rectum & 835 & 28.5 & 4,772 & 30.8 & 16,402 & 45.5 & 111,041 & 38.0 & 134,784 & 38.9 \\
\hline Colon excluding rectum & 547 & 19.7 & 3,073 & 20.3 & 12,256 & 34.6 & 79,012 & 27.1 & 95,962 & 27.8 \\
\hline Rectum and rectosigmoid junction & 288 & 8.8 & 1,699 & 10.5 & 4,146 & 10.9 & 32,029 & 11.0 & 38,822 & 11.1 \\
\hline Anus, anal canal, and anorectum & 33 & 0.9 & 81 & 0.5 & 703 & 1.8 & 5,450 & 1.8 & 6,338 & 1.8 \\
\hline Liver and Intrahepatic bile duct & 280 & 8.5 & 2,008 & 12.7 & 4,097 & 10.0 & 21,223 & 7.0 & 28,012 & 7.7 \\
\hline Gallbladder & 48 & 1.9 & 204 & 1.4 & 558 & 1.6 & 2,979 & 1.0 & 3,835 & 1.1 \\
\hline Other biliary & 28 & 0.9 & 363 & 2.5 & 506 & 1.5 & 5,023 & 1.7 & 5,963 & 1.7 \\
\hline Pancreas & 197 & 7.0 & 1,340 & 9.3 & 5,293 & 15.1 & 36,001 & 12.1 & 43,213 & 12.3 \\
\hline Retroperitoneum & - & - & 43 & 0.3 & 164 & 0.4 & 1,088 & 0.4 & 1,321 & 0.4 \\
\hline $\begin{array}{l}\text { Peritoneum, omentum, and } \\
\text { mesentery }\end{array}$ & - & - & 70 & 0.4 & 135 & 0.4 & 1,698 & 0.6 & 1,924 & 0.5 \\
\hline Other digestive organs & - & - & 80 & 0.5 & 199 & 0.6 & 1,332 & 0.5 & 1,635 & 0.5 \\
\hline Respiratory system & 1,167 & 42.3 & 5,369 & 36.8 & 24,484 & 68.6 & 193,417 & 65.2 & 225,933 & 64.7 \\
\hline Nose, nasal cavity, and middle ear & - & - & 94 & 0.6 & 232 & 0.6 & 1,908 & 0.7 & 2,274 & 0.7 \\
\hline Larynx & 56 & 1.8 & 159 & 1.0 & 1,706 & 4.4 & 10,123 & 3.3 & 12,152 & 3.4 \\
\hline Lung and bronchus & 1,096 & 40.1 & 5,088 & 35.0 & 22,475 & 63.3 & 180,823 & 61.0 & 210,828 & 60.4 \\
\hline Pleura & - & - & - & - & - & - & 91 & 0 & 105 & 0 \\
\hline $\begin{array}{l}\text { Trachea, mediastinum, and other } \\
\text { respiratory organs }\end{array}$ & - & - & 26 & 0.2 & 61 & 0.2 & 472 & 0.2 & 574 & 0.2 \\
\hline Bones and joints & 19 & 0.4 & 109 & 0.6 & 308 & 0.7 & 2,458 & 1.0 & 2,951 & 0.9 \\
\hline Soft tissue including heart & 72 & 2.1 & 356 & 2.1 & 1,308 & 3.4 & 8,809 & 3.2 & 10,728 & 3.2 \\
\hline Skin excluding basal and squamous & 167 & 5.6 & 315 & 2.0 & 664 & 1.8 & 68,552 & 24.2 & 73,181 & 21.5 \\
\hline Melanoma of the skin & 141 & 4.7 & 197 & 1.2 & 332 & 0.9 & 63,875 & 22.6 & 67,753 & 19.9 \\
\hline Other nonepithelial skin & 26 & 0.8 & 118 & 0.8 & 332 & 0.9 & 4,677 & 1.6 & 5,428 & 1.6 \\
\hline Male and female breast & 1,145 & 36.1 & 8,416 & 49.3 & 25,927 & 68.5 & 188,474 & 65.5 & 226,272 & 65.6 \\
\hline Female breast & 1,143 & 67.8 & 8,357 & 89.7 & 25,630 & 120.1 & 186,726 & 123.3 & 224,147 & 122.2 \\
\hline Male breast & - & - & 59 & 0.8 & 297 & 2.0 & 1,748 & 1.3 & 2,125 & 1.4 \\
\hline Female genital system & 598 & 33.9 & 3,279 & 34.9 & 10,015 & 46.9 & 75,241 & 49.4 & 90,303 & 48.8 \\
\hline Cervix & 119 & 6.3 & 576 & 6.1 & 1,907 & 9.0 & 9,174 & 7.1 & 12,042 & 7.4 \\
\hline Corpus and uterus, NOS & 290 & 15.9 & 1,704 & 17.9 & 5,334 & 24.7 & 41,235 & 26.1 & 49,154 & 25.7 \\
\hline Corpus & 281 & 15.3 & 1,648 & 17.3 & 4,995 & 23.1 & 40,082 & 25.3 & 47,570 & 24.9 \\
\hline Uterus, NOS & - & - & 56 & 0.6 & 339 & 1.6 & 1,153 & 0.7 & 1,584 & 0.8 \\
\hline Ovary & 138 & 8.4 & 810 & 8.7 & 1,959 & 9.3 & 17,671 & 11.6 & 20,785 & 11.3 \\
\hline Vagina & - & - & 37 & 0.4 & 199 & 1.0 & 1,031 & 0.7 & 1,296 & 0.7 \\
\hline Vulva & 24 & 1.5 & 66 & 0.8 & 413 & 2.0 & 4,285 & 2.7 & 4,851 & 2.6 \\
\hline Other female genital organs & 16 & 1.0 & 86 & 1.0 & 203 & 1.0 & 1,845 & 1.2 & 2,175 & 1.2 \\
\hline
\end{tabular}

See table footnotes on page 44 . 
TABLE 9. (Continued) Reported number and age-adjusted rate* of invasive ${ }^{\dagger}$ cancer cases, by primary cancer site and race ${ }^{\S}$-United States, $2012^{\uparrow}$

\begin{tabular}{|c|c|c|c|c|c|c|c|c|c|c|}
\hline \multirow[b]{2}{*}{ Cancer site } & \multicolumn{2}{|c|}{$\mathrm{Al} / \mathrm{AN}^{\S}$} & \multicolumn{2}{|c|}{$\mathrm{API}^{\S}$} & \multicolumn{2}{|c|}{ Black } & \multicolumn{2}{|c|}{ White } & \multicolumn{2}{|c|}{ Total } \\
\hline & No. & Rate & No. & Rate & No. & Rate & No. & Rate & No. & Rate \\
\hline Male genital system & 832 & 60.1 & 3,975 & 57.1 & 28,484 & 172.2 & 146,244 & 103.0 & 187,308 & 111.8 \\
\hline Prostate & 750 & 56.4 & 3,755 & 54.5 & 28,003 & 169.4 & 137,488 & 95.6 & 177,489 & 105.3 \\
\hline Testis & 76 & 3.2 & 164 & 1.8 & 305 & 1.5 & 7,410 & 6.3 & 8,189 & 5.5 \\
\hline Penis & - & - & 37 & 0.6 & 142 & 1.0 & 1,070 & 0.8 & 1,283 & 0.8 \\
\hline Other male genital organs & - & - & 19 & 0.3 & 34 & 0.2 & 276 & 0.2 & 347 & 0.2 \\
\hline Urinary system & 710 & 23.9 & 2,443 & 16.4 & 10,399 & 28.8 & 112,781 & 38.4 & 128,103 & 37.0 \\
\hline Urinary bladder & 222 & 8.6 & 1,208 & 8.6 & 3,778 & 11.3 & 63,564 & 21.5 & 69,974 & 20.2 \\
\hline Kidney and renal pelvis & 480 & 15.0 & 1,165 & 7.3 & 6,420 & 16.9 & 46,625 & 16.0 & 55,231 & 15.9 \\
\hline Ureter & - & - & 53 & 0.4 & 80 & 0.2 & 1,769 & 0.6 & 1,918 & 0.6 \\
\hline Other urinary organs & - & - & 17 & 0.1 & 121 & 0.3 & 823 & 0.3 & 980 & 0.3 \\
\hline Eye and orbit & - & - & 36 & 0.2 & 93 & 0.2 & 2,513 & 0.9 & 2,733 & 0.8 \\
\hline Brain and other nervous system & 114 & 3.2 & 622 & 3.6 & 1,615 & 4.1 & 18,858 & 6.9 & 21,490 & 6.5 \\
\hline Brain & 107 & 3.1 & 576 & 3.4 & 1,465 & 3.7 & 17,765 & 6.5 & 20,151 & 6.0 \\
\hline Cranial nerves other nervous system & - & - & 46 & 0.3 & 150 & 0.4 & 1,093 & 0.4 & 1,339 & 0.4 \\
\hline Endocrine system & 285 & 7.4 & 2,822 & 15.6 & 3,893 & 9.9 & 40,662 & 15.6 & 48,594 & 15.0 \\
\hline Thyroid & 275 & 7.2 & 2,677 & 14.8 & 3,529 & 8.9 & 38,888 & 14.9 & 46,279 & 14.3 \\
\hline Other endocrine including thymus & - & - & 145 & 0.8 & 364 & 0.9 & 1,774 & 0.7 & 2,315 & 0.7 \\
\hline Lymphomas & 340 & 11.6 & 2,154 & 13.7 & 6,185 & 16.1 & 61,671 & 21.7 & 71,692 & 21.1 \\
\hline Hodgkin lymphoma & 32 & 0.9 & 212 & 1.1 & 1,096 & 2.6 & 6,787 & 2.7 & 8,273 & 2.6 \\
\hline Non-Hodgkin lymphoma & 308 & 10.8 & 1,942 & 12.5 & 5,089 & 13.5 & 54,884 & 19.0 & 63,419 & 18.5 \\
\hline Myeloma & 116 & 4.1 & 491 & 3.2 & 4,392 & 12.4 & 16,480 & 5.6 & 21,829 & 6.3 \\
\hline Leukemias & 252 & 7.9 & 1,200 & 7.5 & 3,838 & 10.5 & 37,968 & 13.5 & 44,396 & 13.2 \\
\hline Acute lymphocytic leukemia & 51 & 1.2 & 242 & 1.4 & 440 & 1.0 & 4,011 & 1.7 & 4,846 & 1.6 \\
\hline Chronic lymphocytic leukemia & 51 & 1.9 & 172 & 1.1 & 1,066 & 3.1 & 12,921 & 4.3 & 14,821 & 4.2 \\
\hline Acute myeloid leukemia & 86 & 2.8 & 495 & 3.2 & 1,294 & 3.6 & 11,808 & 4.2 & 13,820 & 4.1 \\
\hline Chronic myeloid leukemia & 36 & 1.2 & 177 & 1.1 & 564 & 1.5 & 4,631 & 1.7 & 5,543 & 1.7 \\
\hline Other leukemias & 28 & 0.8 & 114 & 0.7 & 474 & 1.3 & 4,597 & 1.6 & 5,366 & 1.6 \\
\hline Mesothelioma & - & - & 35 & 0.3 & 162 & 0.5 & 2,963 & 1.0 & 3,199 & 0.9 \\
\hline Kaposi Sarcoma & - & - & 27 & 0.1 & 320 & 0.8 & 648 & 0.3 & 1,076 & 0.3 \\
\hline Miscellaneous & 342 & 12.9 & 1,432 & 10.0 & 5,494 & 16.0 & 47,547 & 16.2 & 55,876 & 16.3 \\
\hline
\end{tabular}

Abbreviations: Al/AN = American Indian/Alaska Native; A/PI = Asian/Pacific Islander; NOS = not otherwise specified.

* Rates are the number of cases per 100,000 persons and are age-adjusted to the 2000 U.S. standard population (19 age groups - Census P25-1130). For more

information, see USCS technical notes (http://www.cdc.gov/cancer/npcr/uscs/pdf/uscs-2012-technical-notes.pdf).

${ }^{\dagger}$ Invasive cancer excludes basal and squamous cell carcinomas of the skin except when these occur on the skin of the genital organs, and in situ cancers except urinary bladder. Urinary bladder cancer includes invasive and in situ.

$\S$ Rates are not presented for persons of unknown or other race, therefore categories do not sum to total. Data for specified racial populations other than white and black should be interpreted with caution. For more information, see USCS technical notes (http://www.cdc.gov/cancer/npcr/uscs/pdf/uscs-2012-technical-notes. pdf\#nameddest=IntRaceEthnicityData).

I Data are compiled from cancer registries that meet the data quality criteria for all invasive cancer sites combined (covering approximately $99 \%$ of the U.S. population). Registry-specific data quality information is available at http://www.cdc.gov/cancer/npcr/uscs/pdf/uscs-2012-technical-notes.pdf\#nameddest=RegistriesPubCriteria.

** Counts and rates are suppressed if $<16$ cases were reported. 
TABLE 10. Reported number and age-adjusted rate* of cancer deaths, by primary cancer site and race ${ }^{\dagger}$ United States, $2012^{\S}$

\begin{tabular}{|c|c|c|c|c|c|c|c|c|c|c|}
\hline \multirow[b]{2}{*}{ Cancer site } & \multicolumn{2}{|c|}{${\mathrm{Al} / \mathrm{AN}^{\dagger}}^{+}$} & \multicolumn{2}{|c|}{$\mathrm{API}^{\dagger}$} & \multicolumn{2}{|c|}{ Black } & \multicolumn{2}{|c|}{ White } & \multicolumn{2}{|c|}{ Total } \\
\hline & No. & Rate & No. & Rate & No. & Rate & No. & Rate & No. & Rate \\
\hline All cancer sites combined & 3,018 & 112.5 & 15,339 & 104.2 & 67,374 & 194.4 & 496,876 & 166.4 & 582,607 & 166.4 \\
\hline $\begin{array}{l}\text { Oral cavity and pharynx } \\
\text { Lip }\end{array}$ & 48 & $\begin{array}{l}1.6 \\
-\end{array}$ & 320 & 2.0 & $\begin{array}{r}1,074 \\
-\end{array}$ & 2.9 & $\begin{array}{r}7,482 \\
68\end{array}$ & $\begin{array}{r}2.5 \\
0\end{array}$ & $\begin{array}{r}8,924 \\
71\end{array}$ & $\begin{array}{r}2.5 \\
0\end{array}$ \\
\hline Tongue & - & - & 58 & 0.4 & 200 & 0.5 & 1,956 & 0.6 & 2,224 & 0.6 \\
\hline Salivary gland & - & - & 19 & 0.1 & 67 & 0.2 & 781 & 0.3 & 869 & 0.2 \\
\hline Floor of mouth & - & - & - & - & - & - & 71 & 0 & 75 & 0 \\
\hline Gum and other mouth & - & - & 41 & 0.3 & 118 & 0.3 & 1,108 & 0.4 & 1,275 & 0.4 \\
\hline Nasopharynx & - & - & 141 & 0.8 & 100 & 0.3 & 419 & 0.1 & 666 & 0.2 \\
\hline Tonsil & - & - & - & - & 108 & 0.3 & 753 & 0.2 & 879 & 0.2 \\
\hline Oropharynx & - & - & 17 & 0.1 & 146 & 0.4 & 723 & 0.2 & 891 & 0.2 \\
\hline Hypopharynx & - & - & - & - & 50 & 0.1 & 297 & 0.1 & 356 & 0.1 \\
\hline Other oral cavity and pharynx & - & - & 21 & 0.1 & 281 & 0.8 & 1,306 & 0.4 & 1,618 & 0.4 \\
\hline Digestive system & 908 & 33.1 & 5,511 & 37.5 & 18,930 & 53.9 & 121,675 & 40.5 & 147,024 & 41.6 \\
\hline Esophagus & 67 & 2.5 & 241 & 1.6 & 1,387 & 3.7 & 12,954 & 4.3 & 14,649 & 4.1 \\
\hline Stomach & 100 & 3.9 & 832 & 5.7 & 2,001 & 5.9 & 8,258 & 2.8 & 11,191 & 3.2 \\
\hline Small intestine & - & - & 32 & 0.2 & 199 & 0.5 & 1,060 & 0.4 & 1,293 & 0.4 \\
\hline Colon and rectum & 301 & 11.2 & 1,578 & 10.8 & 6,861 & 19.9 & 42,776 & 14.3 & 51,516 & 14.7 \\
\hline Colon excluding rectum & 246 & 9.2 & 1,270 & 8.8 & 5,727 & 16.8 & 34,624 & 11.6 & 41,867 & 12.0 \\
\hline Rectum and rectosigmoid junction & 55 & 2.0 & 308 & 2.0 & 1,134 & 3.2 & 8,152 & 2.7 & 9,649 & 2.7 \\
\hline Anus, anal canal, and anorectum & - & - & - & - & 99 & 0.3 & 778 & 0.3 & 889 & 0.3 \\
\hline Liver and intrahepatic bile duct & 207 & 7.0 & 1,499 & 9.9 & 3,190 & 8.3 & 18,076 & 5.9 & 22,972 & 6.3 \\
\hline Gallbladder & 24 & 1.1 & 86 & 0.6 & 285 & 0.8 & 1,707 & 0.6 & 2,102 & 0.6 \\
\hline Other biliary & - & - & 61 & 0.4 & 121 & 0.4 & 1,327 & 0.4 & 1,519 & 0.4 \\
\hline Pancreas & 180 & 6.5 & 1,113 & 7.9 & 4,595 & 13.4 & 32,909 & 10.9 & 38,797 & 11.0 \\
\hline Retroperitoneum & - & - & - & - & 16 & 0 & 184 & 0.1 & 207 & 0.1 \\
\hline Peritoneum, omentum, and mesentery & - & - & 18 & 0.1 & 50 & 0.1 & 679 & 0.2 & 750 & 0.2 \\
\hline Other digestive organs & - & - & 37 & 0.3 & 126 & 0.4 & 967 & 0.3 & 1,139 & 0.3 \\
\hline Respiratory system & 818 & 31.1 & 3,528 & 24.6 & 17,484 & 50.3 & 140,021 & 46.9 & 161,851 & 46.2 \\
\hline Nose, nasal cavity, and middle ear & - & - & 19 & 0.1 & 40 & 0.1 & 397 & 0.1 & 458 & 0.1 \\
\hline Larynx & 18 & 0.6 & 56 & 0.4 & 628 & 1.7 & 2,960 & 1.0 & 3,662 & 1.0 \\
\hline Lung and bronchus & 796 & 30.4 & 3,441 & 24.0 & 16,780 & 48.4 & 136,406 & 45.7 & 157,423 & 45.0 \\
\hline Pleura & - & - & - & - & - & - & 75 & 0 & 82 & 0 \\
\hline $\begin{array}{l}\text { Trachea, mediastinum, and other } \\
\text { respiratory organs }\end{array}$ & - & - & - & - & 31 & 0.1 & 183 & 0.1 & 226 & 0.1 \\
\hline Bones and joints & - & - & 42 & 0.3 & 165 & 0.4 & 1,186 & 0.4 & 1,399 & 0.4 \\
\hline Soft tissue including heart & 35 & 1.1 & 147 & 0.9 & 567 & 1.5 & 3,810 & 1.3 & 4,559 & 1.3 \\
\hline Skin excluding basal and squamous & 32 & 1.3 & 90 & 0.6 & 273 & 0.8 & 12,068 & 4.1 & 12,463 & 3.6 \\
\hline Melanoma of the skin & 19 & 0.8 & 58 & 0.4 & 138 & 0.4 & 9,036 & 3.1 & 9,251 & 2.7 \\
\hline Other nonepithelial skin & - & - & 32 & 0.2 & 135 & 0.4 & 3,032 & 1.0 & 3,212 & 0.9 \\
\hline Male and female breast & 173 & 5.9 & 1,036 & 6.3 & 6,246 & 17.2 & 34,100 & 11.4 & 41,555 & 11.8 \\
\hline Female breast & 172 & 10.8 & 1,032 & 11.3 & 6,186 & 29.4 & 33,760 & 20.7 & 41,150 & 21.3 \\
\hline Male breast & - & - & - & - & 60 & 0.4 & 340 & 0.3 & 405 & 0.3 \\
\hline
\end{tabular}

See table footnotes on page 46. 
TABLE 10. (Continued) Reported number and age-adjusted rate* of cancer deaths, by primary cancer site and race ${ }^{\dagger}-U_{\text {nited }}^{*}$ States, $2012^{\S}$

\begin{tabular}{|c|c|c|c|c|c|c|c|c|c|c|}
\hline \multirow[b]{2}{*}{ Cancer site } & \multicolumn{2}{|c|}{ 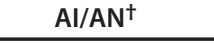 } & \multicolumn{2}{|c|}{$\mathrm{API}^{\dagger}$} & \multicolumn{2}{|c|}{ Black } & \multicolumn{2}{|c|}{ White } & \multicolumn{2}{|c|}{ Total } \\
\hline & No. & Rate & No. & Rate & No. & Rate & No. & Rate & No. & Rate \\
\hline Female genital system & 171 & 11.1 & 858 & 9.6 & 3,984 & 19.4 & 24,392 & 15.0 & 29,405 & 15.2 \\
\hline Cervix & 41 & 2.3 & 154 & 1.7 & 780 & 3.7 & 3,099 & 2.1 & 4,074 & 2.3 \\
\hline Corpus and uterus, NOS & 47 & 3.1 & 280 & 3.2 & 1,666 & 8.2 & 6,918 & 4.1 & 8,911 & 4.5 \\
\hline Corpus & 21 & 1.2 & 131 & 1.5 & 646 & 3.2 & 3,014 & 1.8 & 3,812 & 1.9 \\
\hline Uterus, NOS & 26 & 1.8 & 149 & 1.7 & 1,020 & 5.0 & 3,904 & 2.3 & 5,099 & 2.6 \\
\hline Ovary & 74 & 5.0 & 385 & 4.3 & 1,359 & 6.6 & 12,586 & 7.7 & 14,404 & 7.4 \\
\hline Vagina & - & - & - & - & 56 & 0.3 & 360 & 0.2 & 429 & 0.2 \\
\hline Vulva & - & - & - & - & 64 & 0.3 & 952 & 0.6 & 1,034 & 0.5 \\
\hline Other female genital organs & - & - & 16 & 0.2 & 59 & 0.3 & 477 & 0.3 & 553 & 0.3 \\
\hline Male genital system & 134 & 14.4 & 441 & 8.6 & 4,655 & 42.1 & 22,725 & 18.6 & 27,955 & 20.0 \\
\hline Prostate & 127 & 14.0 & 429 & 8.4 & 4,595 & 41.8 & 22,093 & 18.1 & 27,244 & 19.6 \\
\hline Testis & - & - & - & - & 26 & 0.1 & 347 & 0.3 & 386 & 0.3 \\
\hline Penis & - & - & - & - & 27 & 0.2 & 241 & 0.2 & 273 & 0.2 \\
\hline Other male genital organs & - & - & - & - & - & - & 44 & 0 & 52 & 0 \\
\hline Urinary system & 148 & 5.5 & 507 & 3.7 & 2,411 & 7.2 & 26,528 & 8.8 & 29,594 & 8.5 \\
\hline Urinary bladder & 33 & 1.3 & 232 & 1.8 & 1,090 & 3.5 & 13,890 & 4.6 & 15,245 & 4.4 \\
\hline Kidney and renal pelvis & 107 & 3.9 & 256 & 1.7 & 1,267 & 3.6 & 11,888 & 4.0 & 13,518 & 3.8 \\
\hline Ureter & - & - & - & - & - & - & 351 & 0.1 & 378 & 0.1 \\
\hline Other urinary organs & - & - & - & - & 41 & 0.1 & 399 & 0.1 & 453 & 0.1 \\
\hline Eye and orbit & - & - & - & - & - & - & 260 & 0.1 & 279 & 0.1 \\
\hline Brain and other nervous system & 63 & 2.0 & 336 & 2.1 & 1,009 & 2.7 & 13,868 & 4.8 & 15,276 & 4.4 \\
\hline Endocrine system & - & - & 134 & 0.9 & 311 & 0.9 & 2,208 & 0.8 & 2,660 & 0.8 \\
\hline Thyroid & - & - & 90 & 0.6 & 165 & 0.5 & 1,430 & 0.5 & 1,690 & 0.5 \\
\hline Other endocrine including thymus & - & - & 44 & 0.3 & 146 & 0.4 & 778 & 0.3 & 970 & 0.3 \\
\hline Lymphomas & 75 & 3.2 & 587 & 4.1 & 1,595 & 4.6 & 19,261 & 6.5 & 21,518 & 6.2 \\
\hline Hodgkin lymphoma & - & - & 20 & 0.1 & 133 & 0.3 & 974 & 0.3 & 1,130 & 0.3 \\
\hline Non-Hodgkin lymphoma & 72 & 3.2 & 567 & 4.0 & 1,462 & 4.3 & 18,287 & 6.2 & 20,388 & 5.9 \\
\hline Myeloma & 48 & 2.0 & 241 & 1.7 & 2,085 & 6.3 & 9,447 & 3.2 & 11,821 & 3.4 \\
\hline Leukemias & 96 & 3.4 & 599 & 4.1 & 1,965 & 5.8 & 20,649 & 7.1 & 23,309 & 6.8 \\
\hline Acute lymphocytic leukemia & - & - & 53 & 0.3 & 121 & 0.3 & 1,222 & 0.5 & 1,408 & 0.4 \\
\hline Chronic lymphocytic leukemia & - & - & 38 & 0.3 & 365 & 1.2 & 4,183 & 1.4 & 4,598 & 1.3 \\
\hline Acute myeloid leukemia & 44 & 1.5 & 323 & 2.2 & 768 & 2.2 & 8,349 & 2.9 & 9,484 & 2.8 \\
\hline Chronic myeloid leukemia & - & - & 21 & 0.1 & 108 & 0.3 & 885 & 0.3 & 1,017 & 0.3 \\
\hline Other leukemias & 25 & 1.0 & 164 & 1.2 & 603 & 1.8 & 6,010 & 2.0 & 6,802 & 2.0 \\
\hline Mesothelioma & - & - & 28 & 0.2 & 125 & 0.4 & 2,519 & 0.9 & 2,686 & 0.8 \\
\hline Miscellaneous & 236 & 8.9 & 930 & 6.5 & 4,471 & 12.9 & 34,639 & 11.5 & 40,276 & 11.5 \\
\hline
\end{tabular}

Abbreviations: Al/AN = American Indian/Alaska Native; A/PI = Asian/Pacific Islander; NOS = not otherwise specified.

* Rates are the number of deaths per 100,000 persons and are age-adjusted to the 2000 U.S. standard population (19 age groups - Census P25-1130). For more information, see USCS technical notes (http://www.cdc.gov/cancer/npcr/uscs/pdf/uscs-2012-technical-notes.pdf).

+ Data for specified racial populations other than white and black should be interpreted with caution. For more information, see USCS technical notes (http://www. cdc.gov/cancer/npcr/uscs/pdf/uscs-2012-technical-notes.pdf\#nameddest=IntRaceEthnicityData).

$\S$ Data are from the National Vital Statistics System (NVSS).

" Counts and rates are suppressed if $<16$ deaths were reported. 
TABLE 11. Reported number and age-adjusted rate* of invasive ${ }^{\dagger}$ cancer cases, by primary cancer site and ethnicity ${ }^{\S}$ — United States, $2012^{\Uparrow}$

\begin{tabular}{|c|c|c|c|c|c|c|}
\hline \multirow[b]{2}{*}{ Cancer site } & \multicolumn{2}{|c|}{ Hispanic } & \multicolumn{2}{|c|}{ Non-Hispanic } & \multicolumn{2}{|c|}{ Total } \\
\hline & No. & Rate & No. & Rate & No. & Rate \\
\hline All cancer sites combined & 111,815 & 340.5 & $1,417,061$ & 451.4 & $1,529,078$ & 440.3 \\
\hline Oral cavity and pharynx & 2,318 & 6.9 & 37,561 & 11.7 & 39,879 & 11.2 \\
\hline Lip & 87 & 0.3 & 1,755 & 0.6 & 1,842 & 0.5 \\
\hline Tongue & 629 & 1.9 & 11,745 & 3.6 & 12,374 & 3.4 \\
\hline Salivary gland & 324 & 0.9 & 3,930 & 1.3 & 4,254 & 1.3 \\
\hline Floor of mouth & 90 & 0.3 & 1,846 & 0.6 & 1,936 & 0.5 \\
\hline Gum and other mouth & 366 & 1.2 & 4,965 & 1.6 & 5,331 & 1.5 \\
\hline Nasopharynx & 148 & 0.4 & 1,610 & 0.5 & 1,758 & 0.5 \\
\hline Tonsil & 364 & 1.0 & 6,966 & 2.1 & 7,330 & 2.0 \\
\hline Oropharynx & 97 & 0.3 & 1,636 & 0.5 & 1,733 & 0.5 \\
\hline Hypopharynx & 139 & 0.4 & 2,142 & 0.6 & 2,281 & 0.6 \\
\hline Other oral cavity and pharynx & 74 & 0.2 & 966 & 0.3 & 1,040 & 0.3 \\
\hline Digestive system & 24,117 & 78.3 & 249,370 & 78.5 & 273,535 & 78.3 \\
\hline Esophagus & 847 & 2.8 & 15,143 & 4.7 & 15,993 & 4.5 \\
\hline Stomach & 2,926 & 9.5 & 19,694 & 6.2 & 22,623 & 6.6 \\
\hline Small intestine & 561 & 1.7 & 7,333 & 2.3 & 7,894 & 2.3 \\
\hline Colon and rectum & 10,585 & 34.0 & 124,186 & 39.5 & 134,784 & 38.9 \\
\hline Colon excluding rectum & 7,158 & 23.8 & 88,791 & 28.3 & 95,962 & 27.8 \\
\hline Rectum and rectosigmoid junction & 3,427 & 10.2 & 35,395 & 11.2 & 38,822 & 11.1 \\
\hline Anus, anal canal, and anorectum & 449 & 1.4 & 5,888 & 1.9 & 6,338 & 1.8 \\
\hline Liver and Intrahepatic bile duct & 4,066 & 12.7 & 23,937 & 7.2 & 28,012 & 7.7 \\
\hline Gallbladder & 539 & 1.9 & 3,295 & 1.0 & 3,835 & 1.1 \\
\hline Other biliary & 650 & 2.2 & 5,313 & 1.7 & 5,963 & 1.7 \\
\hline Pancreas & 3,079 & 10.7 & 40,116 & 12.5 & 43,213 & 12.3 \\
\hline Retroperitoneum & 128 & 0.3 & 1,193 & 0.4 & 1,321 & 0.4 \\
\hline Peritoneum, omentum, and mesentery & 134 & 0.4 & 1,790 & 0.6 & 1,924 & 0.5 \\
\hline Other digestive organs & 153 & 0.5 & 1,482 & 0.5 & 1,635 & 0.5 \\
\hline Respiratory system & 9,655 & 34.4 & 216,231 & 67.5 & 225,933 & 64.7 \\
\hline Nose, nasal cavity, and middle ear & 191 & 0.6 & 2,083 & 0.7 & 2,274 & 0.7 \\
\hline Larynx & 736 & 2.4 & 11,413 & 3.5 & 12,152 & 3.4 \\
\hline Lung and bronchus & 8,648 & 31.2 & 202,136 & 63.2 & 210,828 & 60.4 \\
\hline Pleura & - $^{* *}$ & - & 101 & 0 & - & 0 \\
\hline Trachea, mediastinum, and other respiratory organs & 76 & 0.1 & 498 & 0.2 & 574 & 0.2 \\
\hline Bones and joints & 415 & 0.9 & 2,536 & 0.9 & 2,951 & 0.9 \\
\hline Soft tissue including heart & 1,092 & 2.8 & 9,636 & 3.3 & 10,728 & 3.2 \\
\hline Skin excluding basal and squamous & 1,726 & 5.1 & 71,452 & 23.6 & 73,181 & 21.5 \\
\hline Melanoma of the skin & 1,423 & 4.2 & 66,328 & 21.9 & 67,753 & 19.9 \\
\hline Other nonepithelial skin & 303 & 0.9 & 5,124 & 1.7 & 5,428 & 1.6 \\
\hline Male and female breast & 17,129 & 49.0 & 209,135 & 67.6 & 226,272 & 65.6 \\
\hline Female breast & 17,014 & 91.3 & 207,125 & 125.9 & 224,147 & 122.2 \\
\hline Male breast & 115 & 0.8 & 2,010 & 1.4 & 2,125 & 1.4 \\
\hline Female genital system & 8,557 & 44.5 & 81,736 & 49.1 & 90,303 & 48.8 \\
\hline Cervix & 1,998 & 9.5 & 10,044 & 7.1 & 12,042 & 7.4 \\
\hline Corpus and uterus, NOS & 4,135 & 21.8 & 45,015 & 26.0 & 49,154 & 25.7 \\
\hline Corpus & 3,976 & 20.9 & 43,593 & 25.2 & 47,570 & 24.9 \\
\hline Uterus, NOS & 159 & 0.9 & 1,422 & 0.8 & 1,584 & 0.8 \\
\hline Ovary & 1,885 & 10.0 & 18,894 & 11.4 & 20,785 & 11.3 \\
\hline Vagina & 108 & 0.6 & 1,188 & 0.7 & 1,296 & 0.7 \\
\hline Vulva & 268 & 1.7 & 4,583 & 2.7 & 4,851 & 2.6 \\
\hline Other female genital organs & 163 & 0.9 & 2,012 & 1.2 & 2,175 & 1.2 \\
\hline
\end{tabular}

See table footnotes on page 48 . 
TABLE 11. (Continued) Reported number and age-adjusted rate* of invasive ${ }^{\dagger}$ cancer cases, by primary cancer site and ethnicity — United $^{\S}$ States, $2012^{\text {? }}$

\begin{tabular}{|c|c|c|c|c|c|c|}
\hline \multirow[b]{2}{*}{ Cancer site } & \multicolumn{2}{|c|}{ Hispanic } & \multicolumn{2}{|c|}{ Non-Hispanic } & \multicolumn{2}{|c|}{ Total } \\
\hline & No. & Rate & No. & Rate & No. & Rate \\
\hline Male genital system & 13,653 & 95.7 & 173,628 & 113.7 & 187,308 & 111.8 \\
\hline Prostate & 12,121 & 89.8 & 165,342 & 107.0 & 177,489 & 105.3 \\
\hline Testis & 1,311 & 4.4 & 6,877 & 5.7 & 8,189 & 5.5 \\
\hline Penis & 204 & 1.4 & 1,079 & 0.8 & 1,283 & 0.8 \\
\hline Other male genital organs & 17 & 0.1 & 330 & 0.2 & 347 & 0.2 \\
\hline Urinary system & 8,518 & 27.4 & 119,571 & 37.9 & 128,103 & 37.0 \\
\hline Urinary bladder & 3,041 & 11.1 & 66,925 & 21.1 & 69,974 & 20.2 \\
\hline Kidney and renal pelvis & 5,350 & 15.9 & 49,875 & 15.9 & 55,231 & 15.9 \\
\hline Ureter & 80 & 0.3 & 1,838 & 0.6 & 1,918 & 0.6 \\
\hline Other urinary organs & 47 & 0.2 & 933 & 0.3 & 980 & 0.3 \\
\hline Eye and orbit & 206 & 0.5 & 2,527 & 0.9 & 2,733 & 0.8 \\
\hline Brain and other nervous system & 1,990 & 4.9 & 19,497 & 6.7 & 21,490 & 6.5 \\
\hline Brain & 1,846 & 4.6 & 18,302 & 6.3 & 20,151 & 6.0 \\
\hline Cranial nerves other nervous system & 144 & 0.3 & 1,195 & 0.5 & 1,339 & 0.4 \\
\hline Endocrine system & 5,806 & 13.7 & 42,783 & 15.4 & 48,594 & 15.0 \\
\hline Thyroid & 5,555 & 13.1 & 40,722 & 14.6 & 46,279 & 14.3 \\
\hline Other endocrine including thymus & 251 & 0.6 & 2,061 & 0.7 & 2,315 & 0.7 \\
\hline Lymphomas & 6,462 & 18.9 & 65,222 & 21.5 & 71,692 & 21.1 \\
\hline Hodgkin lymphoma & 1,029 & 2.3 & 7,244 & 2.7 & 8,273 & 2.6 \\
\hline Non-Hodgkin lymphoma & 5,433 & 16.6 & 57,978 & 18.7 & 63,419 & 18.5 \\
\hline Myeloma & 1,869 & 6.2 & 19,955 & 6.3 & 21,829 & 6.3 \\
\hline Leukemias & 3,988 & 10.3 & 40,403 & 13.4 & 44,396 & 13.2 \\
\hline Acute lymphocytic leukemia & 1,264 & 2.2 & 3,582 & 1.5 & 4,846 & 1.6 \\
\hline Chronic lymphocytic leukemia & 542 & 1.9 & 14,278 & 4.5 & 14,821 & 4.2 \\
\hline Acute myeloid leukemia & 1,272 & 3.6 & 12,546 & 4.1 & 13,820 & 4.1 \\
\hline Chronic myeloid leukemia & 490 & 1.4 & 5,051 & 1.7 & 5,543 & 1.7 \\
\hline Other leukemias & 420 & 1.2 & 4,946 & 1.6 & 5,366 & 1.6 \\
\hline Mesothelioma & 200 & 0.7 & 2,997 & 1.0 & 3,199 & 0.9 \\
\hline Kaposi Sarcoma & 198 & 0.5 & 878 & 0.3 & 1,076 & 0.3 \\
\hline Miscellaneous & 3,916 & 13.6 & 51,943 & 16.5 & 55,876 & 16.3 \\
\hline
\end{tabular}

Abbreviation: NOS = not otherwise specified.

* Rates are the number of cases per 100,000 persons and are age-adjusted to the 2000 U.S. standard population (19 age groups - Census P25-1130). For more information, see USCS technical notes (http://www.cdc.gov/cancer/npcr/uscs/pdf/uscs-2012-technical-notes.pdf).

† Invasive cancer excludes basal and squamous cell carcinomas of the skin except when these occur on the skin of the genital organs, and in situ cancers except urinary bladder. Urinary bladder cancer includes invasive and in situ.

$\S$ Rates and counts are not presented for persons of unknown ethnicity, therefore categories do not sum to total. Data for specified ethnic populations should be interpreted with caution. For more information, see USCS technical notes http://www.cdc.gov/cancer/npcr/uscs/pdf/uscs-2012-technical-notes.pdf\#nameddest= IntRaceEthnicityData).

I Data are compiled from cancer registries that meet the data quality criteria for all invasive cancer sites combined (covering approximately $99 \%$ of the U.S. population). Registry-specific data quality information is available at http://www.cdc.gov/cancer/npcr/uscs/pdf/uscs-2012-technical-notes.pdf\#nameddest=RegistriesPubCriteria.

** Counts and rates are suppressed if $<16$ cases were reported. Some counts and rates are suppressed as complementary cell suppression. 
TABLE 12. Reported number and age-adjusted rate* of cancer deaths, by primary cancer site and ethnicity ${ }^{\dagger}$ United States, $2012^{\S}$

\begin{tabular}{|c|c|c|c|c|c|c|}
\hline \multirow[b]{2}{*}{ Cancer site } & \multicolumn{2}{|c|}{ Hispanic $^{\S}$} & \multicolumn{2}{|c|}{ Non-Hispanic } & \multicolumn{2}{|c|}{ Total } \\
\hline & No. & Rate & No. & Rate & No. & Rate \\
\hline All cancer sites combined & 34,146 & 117.8 & 547,027 & 170.4 & 582,607 & 166.4 \\
\hline Oral cavity and pharynx & 476 & 1.6 & 8,421 & 2.6 & 8,924 & 2.5 \\
\hline Lip & - & - & 65 & 0 & 71 & 0 \\
\hline Tongue & 118 & 0.4 & 2,099 & 0.6 & 2,224 & 0.6 \\
\hline Salivary gland & 48 & 0.2 & 819 & 0.3 & 869 & 0.2 \\
\hline Floor of mouth & - & - & 68 & 0 & 75 & 0 \\
\hline Gum and other mouth & 66 & 0.2 & 1,206 & 0.4 & 1,275 & 0.4 \\
\hline Nasopharynx & 37 & 0.1 & 627 & 0.2 & 666 & 0.2 \\
\hline Tonsil & 39 & 0.1 & 835 & 0.3 & 879 & 0.2 \\
\hline Oropharynx & 43 & 0.1 & 848 & 0.3 & 891 & 0.2 \\
\hline Hypopharynx & 25 & 0.1 & 329 & 0.1 & 356 & 0.1 \\
\hline Other oral cavity and pharynx & 87 & 0.3 & 1,525 & 0.5 & 1,618 & 0.4 \\
\hline Digestive system & 11,382 & 39.3 & 135,287 & 41.8 & 147,024 & 41.6 \\
\hline Esophagus & 621 & 2.1 & 13,995 & 4.3 & 14,649 & 4.1 \\
\hline Stomach & 1,590 & 5.2 & 9,570 & 3.0 & 11,191 & 3.2 \\
\hline Small intestine & 68 & 0.2 & 1,221 & 0.4 & 1,293 & 0.4 \\
\hline Colon and rectum & 3,349 & 11.8 & 48,034 & 15.0 & 51,516 & 14.7 \\
\hline Colon excluding rectum & 2,737 & 9.7 & 39,017 & 12.2 & 41,867 & 12.0 \\
\hline Rectum and rectosigmoid junction & 612 & 2.0 & 9,017 & 2.8 & 9,649 & 2.7 \\
\hline Anus, anal canal, and anorectum & 40 & 0.1 & 845 & 0.3 & 889 & 0.3 \\
\hline Liver and intrahepatic bile duct & 2,780 & 9.3 & 20,135 & 6.1 & 22,972 & 6.3 \\
\hline Gallbladder & 262 & 1.0 & 1,833 & 0.6 & 2,102 & 0.6 \\
\hline Other biliary & 136 & 0.5 & 1,383 & 0.4 & 1,519 & 0.4 \\
\hline Pancreas & 2,407 & 8.6 & 36,307 & 11.2 & 38,797 & 11.0 \\
\hline Retroperitoneum & 16 & 0 & 190 & 0.1 & 207 & 0.1 \\
\hline Peritoneum, omentum, and mesentery & 46 & 0.1 & 703 & 0.2 & 750 & 0.2 \\
\hline Other digestive organs & 67 & 0.2 & 1,071 & 0.3 & 1,139 & 0.3 \\
\hline Respiratory system & 5,503 & 20.4 & 155,925 & 48.4 & 161,851 & 46.2 \\
\hline Nose, nasal cavity, and middle ear & 37 & 0.1 & 420 & 0.1 & 458 & 0.1 \\
\hline Larynx & 214 & 0.8 & 3,433 & 1.0 & 3,662 & 1.0 \\
\hline Lung and bronchus & 5,225 & 19.4 & 151,793 & 47.1 & 157,423 & 45.0 \\
\hline Pleura & - & - & 77 & 0 & 82 & 0 \\
\hline Trachea, mediastinum, and other respiratory organs & 22 & 0.1 & 202 & 0.1 & 226 & 0.1 \\
\hline Bones and joints & 136 & 0.3 & 1,262 & 0.4 & 1,399 & 0.4 \\
\hline Soft tissue including heart & 390 & 1.1 & 4,155 & 1.4 & 4,559 & 1.3 \\
\hline Skin excluding basal and squamous & 350 & 1.2 & 12,085 & 3.8 & 12,463 & 3.6 \\
\hline Melanoma of the skin & 224 & 0.7 & 9,010 & 2.9 & 9,251 & 2.7 \\
\hline Other nonepithelial skin & 126 & 0.5 & 3,075 & 0.9 & 3,212 & 0.9 \\
\hline Male and female breast & 2,627 & 8.1 & 38,826 & 12.1 & 41,555 & 11.8 \\
\hline Female breast & 2,613 & 14.7 & 38,436 & 21.9 & 41,150 & 21.3 \\
\hline Male breast & - & - & 390 & 0.3 & 405 & 0.3 \\
\hline
\end{tabular}

See table footnotes on page 50 . 
TABLE 12. (Continued) Reported number and age-adjusted rate* of cancer deaths, by primary cancer site and ethnicity ${ }^{\dagger}-$ United $^{*}$ tates, $2012^{\S}$

\begin{tabular}{|c|c|c|c|c|c|c|}
\hline \multirow[b]{2}{*}{ Cancer site } & \multicolumn{2}{|c|}{ Hispanic $^{\S}$} & \multicolumn{2}{|c|}{ Non-Hispanic } & \multicolumn{2}{|c|}{ Total } \\
\hline & No. & Rate & No. & Rate & No. & Rate \\
\hline Female genital system & 2,212 & 12.8 & 27,113 & 15.4 & 29,405 & 15.2 \\
\hline Cervix & 520 & 2.7 & 3,536 & 2.2 & 4,074 & 2.3 \\
\hline Corpus and uterus, NOS & 619 & 3.7 & 8,269 & 4.6 & 8,911 & 4.5 \\
\hline Corpus & 245 & 1.5 & 3,555 & 2.0 & 3,812 & 1.9 \\
\hline Uterus, NOS & 374 & 2.2 & 4,714 & 2.6 & 5,099 & 2.6 \\
\hline Ovary & 963 & 5.8 & 13,407 & 7.5 & 14,404 & 7.4 \\
\hline Vagina & 32 & 0.2 & 396 & 0.2 & 429 & 0.2 \\
\hline Vulva & 45 & 0.3 & 986 & 0.5 & 1,034 & 0.5 \\
\hline Other female genital organs & 33 & 0.2 & 519 & 0.3 & 553 & 0.3 \\
\hline Male genital system & 1,706 & 17.1 & 26,189 & 20.2 & 27,955 & 20.0 \\
\hline Prostate & 1,592 & 16.5 & 25,595 & 19.8 & 27,244 & 19.6 \\
\hline Testis & 75 & 0.2 & 309 & 0.2 & 386 & 0.3 \\
\hline Penis & 36 & 0.3 & 237 & 0.2 & 273 & 0.2 \\
\hline Other male genital organs & - & - & 48 & 0 & 52 & 0 \\
\hline Urinary system & 1,660 & 6.0 & 27,875 & 8.6 & 29,594 & 8.5 \\
\hline Urinary bladder & 584 & 2.3 & 14,630 & 4.5 & 15,245 & 4.4 \\
\hline Kidney and renal pelvis & 1,042 & 3.6 & 12,451 & 3.8 & 13,518 & 3.8 \\
\hline Ureter & - & - & 362 & 0.1 & 378 & 0.1 \\
\hline Other urinary organs & 19 & 0.1 & 432 & 0.1 & 453 & 0.1 \\
\hline Eye and orbit & 18 & 0.1 & 260 & 0.1 & 279 & 0.1 \\
\hline Brain and other nervous system & 1,049 & 3.0 & 14,194 & 4.6 & 15,276 & 4.4 \\
\hline Endocrine system & 248 & 0.8 & 2,409 & 0.8 & 2,660 & 0.8 \\
\hline Thyroid & 168 & 0.6 & 1,521 & 0.5 & 1,690 & 0.5 \\
\hline Other endocrine including thymus & 80 & 0.2 & 888 & 0.3 & 970 & 0.3 \\
\hline Lymphomas & 1,551 & 5.4 & 19,914 & 6.3 & 21,518 & 6.2 \\
\hline Hodgkin lymphoma & 123 & 0.4 & 1,005 & 0.3 & 1,130 & 0.3 \\
\hline Non-Hodgkin lymphoma & 1,428 & 5.0 & 18,909 & 6.0 & 20,388 & 5.9 \\
\hline Myeloma & 782 & 2.9 & 11,019 & 3.4 & 11,821 & 3.4 \\
\hline Leukemias & 1,603 & 5.0 & 21,661 & 6.9 & 23,309 & 6.8 \\
\hline Acute lymphocytic leukemia & 325 & 0.7 & 1,080 & 0.4 & 1,408 & 0.4 \\
\hline Chronic lymphocytic leukemia & 125 & 0.5 & 4,463 & 1.4 & 4,598 & 1.3 \\
\hline Acute myeloid leukemia & 582 & 1.9 & 8,885 & 2.9 & 9,484 & 2.8 \\
\hline Chronic myeloid leukemia & 76 & 0.3 & 936 & 0.3 & 1,017 & 0.3 \\
\hline Other leukemias & 495 & 1.7 & 6,297 & 2.0 & 6,802 & 2.0 \\
\hline Mesothelioma & 132 & 0.5 & 2,545 & 0.8 & 2,686 & 0.8 \\
\hline Miscellaneous & 2,313 & 8.2 & 37,842 & 11.7 & 40,276 & 11.5 \\
\hline
\end{tabular}

Abbreviation: NOS = not otherwise specified.

* Rates are the number of deaths per 100,000 persons and are age-adjusted to the 2000 U.S. standard population (19 age groups - Census P25-1130). For more information, see USCS technical notes (http://www.cdc.gov/cancer/npcr/uscs/pdf/uscs-2012-technical-notes.pdf).

${ }^{\dagger}$ Data are from the National Vital Statistics System (NVSS).

$\S$ Data for specified ethnic populations should be interpreted with caution. For more information, see USCS technical notes (http://www.cdc.gov/cancer/npcr/uscs/ pdf/uscs-2012-technical-notes.pdf\#nameddest=IntRaceEthnicityData).

" Counts and rates are suppressed if $<16$ deaths were reported. 
TABLE 13. Reported number of invasive* cancer cases by primary cancer site and year - United States, 1999-2012 ${ }^{\dagger}$

\begin{tabular}{|c|c|c|c|c|c|c|c|c|c|c|c|c|c|c|}
\hline Cancer site & 1999 & 2000 & 2001 & 2002 & 2003 & 2004 & 2005 & 2006 & 2007 & 2008 & 2009 & 2010 & 2011 & 2012 \\
\hline All cancer sites combined & $1,227,562$ & 249,559 & 273,017 & 285,618 & 279,510 & 296,877 & $1,318,81$ & 349,590 & 387,116 & 402,567 & 414,11 & 403,565 & 424,734 & $1,399,978$ \\
\hline Oral cavity and pharynx & 27,491 & 27,954 & 28,236 & 28,904 & 29,506 & 29,980 & 30,315 & 31,314 & 32,798 & 34,214 & 34,619 & 35,395 & 36,790 & 36,990 \\
\hline Lip & 2,444 & 2,393 & 2,309 & 2,333 & 2,041 & 1,961 & 1,859 & 1,880 & 1,883 & 1,867 & 1,832 & 1,952 & 1,886 & 1,725 \\
\hline Tongue & 6,640 & 6,859 & 7,017 & 7,478 & 7,737 & 8,091 & 8,298 & 8,725 & 9,399 & 10,100 & 10,224 & 10,282 & 11,118 & 11,476 \\
\hline Salivary gland & 2,982 & 3,147 & 3,180 & 3,220 & 3,305 & 3,357 & 3,470 & 3,599 & 3,628 & 3,754 & 3,774 & 3,762 & 3,792 & 3,986 \\
\hline Floor of mouth & 2,074 & 2,053 & 2,075 & 2,018 & 1,940 & 1,916 & 1,827 & 1,944 & 1,923 & 1,934 & 1,920 & 1,924 & 1,838 & 1,787 \\
\hline Gum and other mouth & 4,357 & 4,197 & 4,312 & 4,236 & 4,387 & 4,348 & 4,338 & 4,372 & 4,564 & 4,759 & 4,663 & 4,846 & 4,927 & 4,915 \\
\hline Nasopharynx & 1,471 & 1,527 & 1,416 & 1,535 & 1,622 & 1,559 & 1,618 & 1,565 & 1,722 & 1,713 & 1,601 & 1,660 & 1,635 & 1,640 \\
\hline Tonsil & 3,409 & 3,584 & 3,780 & 4,032 & 4,316 & 4,570 & 4,756 & 5,068 & 5,307 & 5,730 & 6,263 & 6,348 & 6,754 & 6,786 \\
\hline Oropharynx & 995 & 1,060 & 1,106 & 1,110 & 1,277 & 1,295 & 1,298 & 1,404 & 1,416 & 1,479 & 1,460 & 1,666 & 1,704 & 1,598 \\
\hline Hypopharynx & 2,187 & 2,153 & 2,085 & 2,066 & 2,163 & 2,131 & 2,120 & 2,034 & 2,131 & 2,082 & 2,127 & 2,074 & 2,124 & 2,106 \\
\hline Other oral cavity and pharynx & 932 & 981 & 956 & 876 & 718 & 752 & 731 & 723 & 825 & 796 & 755 & 881 & 1,012 & 971 \\
\hline Digestive system & 232,437 & 235,790 & 238,113 & 239,050 & 242,294 & 243,671 & 244,545 & 246,111 & 249,358 & 252,542 & 252,284 & 250,837 & 254,183 & 254,508 \\
\hline Esophagus & 12,691 & 12,886 & 13,082 & 13,105 & 13,606 & 14,152 & 14,058 & 14,522 & 14,535 & 15,092 & 15,220 & 14,598 & 14,998 & 14,884 \\
\hline Stomach & 19,350 & 19,227 & 19,184 & 19,418 & 19,689 & 19,653 & 19,392 & 19,400 & 19,827 & 19,674 & 20,036 & 20,925 & 21,054 & 21,168 \\
\hline Small intestine & 4,312 & 4,314 & 4,614 & 4,882 & 5,038 & 5,379 & 5,593 & 5,974 & 6,179 & 6,502 & 6,706 & 7,124 & 7,188 & 7,336 \\
\hline Colon and rectum & 142,816 & 144,059 & 143,905 & 142,685 & 142,037 & 140,094 & 138,690 & 136,944 & 136,405 & 135,443 & 131,492 & 127,515 & 127,219 & 124,944 \\
\hline Colon excluding rectum & 104,182 & 105,255 & 105,235 & 104,439 & 103,760 & 102,098 & 100,503 & 99,464 & 98,772 & 98,055 & 94,444 & 91,344 & 91,134 & 88,810 \\
\hline Rectum and rectosigmoid junction & 38,634 & 38,804 & 38,670 & 38,246 & 38,277 & 37,996 & 38,187 & 37,480 & 37,633 & 37,388 & 37,048 & 36,171 & 36,085 & 36,134 \\
\hline Anus, anal canal, and anorectum & 3,467 & 3,541 & 3,626 & 3,801 & 4,198 & 4,314 & 4,506 & 4,503 & 4,967 & 5,230 & 5,614 & 5,442 & 5,694 & 5,876 \\
\hline Liver and Intrahepatic bile duct & 12,470 & 13,415 & 13,502 & 14,451 & 15,221 & 16,460 & 17,381 & 18,426 & 20,097 & 21,264 & 23,130 & 23,862 & 25,028 & 26,279 \\
\hline Gallbladder & 3,102 & 2,992 & 3,149 & 2,980 & 3,122 & 3,140 & 3,231 & 3,279 & 3,314 & 3,330 & 3,550 & 3,538 & 3,607 & 3,614 \\
\hline Other biliary & 3,356 & 3,544 & 4,221 & 4,401 & 4,565 & 4,624 & 4,677 & 4,862 & 4,874 & 5,141 & 5,349 & 5,437 & 5,635 & 5,603 \\
\hline Pancreas & 28,024 & 28,801 & 29,421 & 29,804 & 31,085 & 31,922 & 32,985 & 34,137 & 34,961 & 36,519 & 36,950 & 38,112 & 39,242 & 40,229 \\
\hline Retroperitoneum & 990 & 963 & 1,033 & 1,062 & 1,038 & 1,057 & 1,125 & 1,094 & 1,114 & 1,136 & 1,135 & 1,151 & 1,227 & 1,257 \\
\hline $\begin{array}{l}\text { Peritoneum, omentum, and } \\
\text { mesentery }\end{array}$ & 1,101 & 1,188 & 1,391 & 1,472 & 1,646 & 1,818 & 1,813 & 1,751 & 1,895 & 1,981 & 1,838 & 1,808 & 1,835 & 1,791 \\
\hline Other digestive organs & 758 & 860 & 985 & 989 & 1,049 & 1,058 & 1,094 & 1,219 & 1,190 & 1,230 & 1,264 & 1,325 & 1,456 & 1,527 \\
\hline Respiratory system & 193,612 & 194,368 & 196,473 & 198,631 & 201,452 & 202,402 & 206,455 & 207,257 & 208,946 & 211,173 & 211,521 & 70 & 207,967 & 207,627 \\
\hline Nose, nasal cavity, and middle ear & 1,805 & 1,799 & 1,765 & 1,870 & 1,815 & 1,928 & 1,966 & 1,953 & 2,116 & 2,080 & 2,104 & 2,005 & 2,045 & 2,126 \\
\hline Larynx & 11,696 & 11,513 & 11,409 & 11,081 & 11,202 & 11,427 & 11,373 & 11,457 & 11,507 & 11,489 & 11,499 & 11,627 & 11,400 & 11,106 \\
\hline Lung and bronchus & 179,444 & 180,405 & 182,729 & 185,079 & 187,813 & 188,372 & 192,465 & 193,219 & 194,673 & 196,903 & 197,245 & 194,261 & 193,906 & 193,768 \\
\hline Pleura & 104 & 101 & 77 & 97 & 92 & 98 & 93 & 83 & 102 & 99 & 104 & 103 & 92 & 100 \\
\hline $\begin{array}{l}\text { Trachea, mediastinum, and other } \\
\text { respiratory organs }\end{array}$ & 563 & 550 & 493 & 504 & 530 & 577 & 558 & 545 & 548 & 602 & 569 & 574 & 524 & 527 \\
\hline Bones and joints & 2,472 & 2,462 & 2,627 & 2,645 & 2,577 & 2,640 & 2,752 & 2,692 & 2,755 & 2,687 & 2,808 & 2,708 & 2,766 & 2,769 \\
\hline Soft tissue including heart & 7,333 & 7,687 & 7,846 & 8,035 & 8,383 & 8,792 & 9,025 & 8,897 & 9,449 & 9,589 & 9,707 & 9,978 & 9,810 & 10,000 \\
\hline $\begin{array}{l}\text { Skin excluding basal and } \\
\text { squamous }\end{array}$ & 42,231 & 45,624 & 49,078 & 50,756 & 51,026 & 54,246 & 58,466 & 58,591 & 60,959 & 63,467 & 65,477 & 65,306 & 67,704 & 68,633 \\
\hline Melanoma of the skin & 38,867 & 41,925 & 45,141 & 46,757 & 46,935 & 50,040 & 54,096 & 54,225 & 56,303 & 58,484 & 60,335 & 60,397 & 62,674 & 63,502 \\
\hline Other nonepithelial skin & 3,364 & 3,699 & 3,937 & 3,999 & 4,091 & 4,206 & 4,370 & 4,366 & 4,656 & 4,983 & 5,142 & 4,909 & 5,030 & 5,131 \\
\hline Male and female breast & 187,922 & 187,408 & 189,541 & 188,438 & 181,949 & 182,828 & 185,308 & 189,451 & 194,935 & 200,299 & 205,221 & 201,894 & 208,322 & 210,356 \\
\hline Female breast & 186,459 & 185,946 & 188,139 & 186,934 & 180,386 & 181,216 & 183,738 & 187,819 & 193,211 & 198,411 & 203,405 & 199,991 & 206,446 & 208,404 \\
\hline Male breast & 1,463 & 1,462 & 1,402 & 1,504 & 1,563 & 1,612 & 1,570 & 1,632 & 1,724 & 1,888 & 1,816 & 1,903 & 1,876 & 1,952 \\
\hline Female genital system & 71,591 & 72,193 & 72,986 & 72,698 & 71,893 & 72,881 & 74,556 & 75,675 & 77,446 & 79,354 & 81,185 & 81,437 & 83,020 & 84,199 \\
\hline Cervix & 12,991 & 12,984 & 12,399 & 12,079 & 11,726 & 11,491 & 11,719 & 11,653 & 11,705 & 11,623 & 11,807 & 11,395 & 11,339 & 11,145 \\
\hline Corpus and uterus, NOS & 33,458 & 33,501 & 34,731 & 34,927 & 34,388 & 35,868 & 36,928 & 37,859 & 39,260 & 40,755 & 42,496 & 43,347 & 44,709 & 45,902 \\
\hline Corpus & 32,350 & 32,366 & 33,573 & 33,763 & 33,224 & 34,727 & 35,751 & 36,721 & 38,124 & 39,440 & 41,081 & 41,970 & 43,247 & 44,449 \\
\hline Uterus, NOS & 1,108 & 1,135 & 1,158 & 1,164 & 1,164 & 1,141 & 1,177 & 1,138 & 1,136 & 1,315 & 1,415 & 1,377 & 1,462 & 1,453 \\
\hline Ovary & 19,875 & 20,339 & 20,361 & 20,075 & 20,187 & 19,805 & 20,113 & 20,175 & 20,131 & 20,409 & 20,000 & 19,569 & 19,524 & 19,382 \\
\hline Vagina & 1,020 & 1,088 & 1,075 & 1,100 & 1,052 & 1,103 & 1,010 & 1,083 & 1,097 & 1,146 & 1,154 & 1,163 & 1,178 & 1,199 \\
\hline Vulva & 3,401 & 3,348 & 3,494 & 3,515 & 3,551 & 3,579 & 3,700 & 3,788 & 3,986 & 4,017 & 4,201 & 4,223 & 4,355 & 4,502 \\
\hline Other female genital organs & 846 & 933 & 926 & 1,002 & 989 & 1,035 & 1,086 & 1,117 & 1,267 & 1,404 & 1,527 & 1,740 & 1,915 & 2,069 \\
\hline
\end{tabular}

See table footnotes on page 52 . 
TABLE 13. Reported number of invasive* cancer cases by primary cancer site and year - United States, 1999-2012 ${ }^{\dagger}$

\begin{tabular}{|c|c|c|c|c|c|c|c|c|c|c|c|c|c|c|}
\hline Cancer site & 1999 & 2000 & 2001 & 2002 & 2003 & 2004 & 2005 & 2006 & 2007 & 2008 & 2009 & 2010 & 2011 & 2012 \\
\hline Male genital system & 194,920 & 200,489 & 206,681 & 208,978 & 196,114 & 195,842 & 196,522 & 214,202 & 226,520 & 215,968 & 211,749 & 203,731 & 207,234 & 173,864 \\
\hline Prostate & 186,717 & 192,151 & 198,257 & 200,678 & 187,529 & 187,229 & 187,731 & 205,526 & 217,631 & 206,730 & 202,691 & 194,600 & 198,110 & 164,613 \\
\hline Testis & 6,937 & 7,052 & 7,160 & 7,017 & 7,251 & 7,355 & 7,501 & 7,346 & 7,509 & 7,690 & 7,650 & 7,673 & 7,579 & 7,724 \\
\hline Penis & 1,004 & 1,008 & 991 & 1,025 & 1,053 & 988 & 997 & 1,025 & 1,099 & 1,211 & 1,106 & 1,143 & 1,206 & 1,194 \\
\hline Other male genital organs & 262 & 278 & 273 & 258 & 281 & 270 & 293 & 305 & 281 & 337 & 302 & 315 & 339 & 333 \\
\hline Urinary system & 89,166 & 91,902 & 94,178 & 97,018 & 100,211 & 103,654 & 106,689 & 108,167 & 111,587 & 114,459 & 115,944 & 115,668 & 117,650 & 119,465 \\
\hline Urinary bladder & 55,822 & 56,845 & 57,153 & 58,168 & 59,242 & 60,732 & 61,706 & 60,721 & 62,484 & 63,354 & 64,138 & 64,051 & 64,872 & 65,494 \\
\hline Kidney and renal pelvis & 31,073 & 32,824 & 34,756 & 36,629 & 38,706 & 40,615 & 42,661 & 44,965 & 46,608 & 48,383 & 49,203 & 49,018 & 50,068 & 51,269 \\
\hline Ureter & 1,553 & 1,536 & 1,560 & 1,565 & 1,573 & 1,608 & 1,600 & 1,721 & 1,668 & 1,701 & 1,723 & 1,720 & 1,764 & 1,783 \\
\hline Other urinary organs & 718 & 697 & 709 & 656 & 690 & 699 & 722 & 760 & 827 & 1,021 & 880 & 879 & 946 & 919 \\
\hline Eye and orbit & 2,185 & 2,266 & 2,283 & 2,258 & 2,441 & 2,459 & 2,440 & 2,484 & 2,538 & 2,514 & 2,537 & 2,438 & 2,474 & 2,589 \\
\hline Brain and other nervous system & 17,611 & 17,830 & 17,766 & 18,267 & 18,353 & 18 & 18,993 & 19,201 & 19,512 & 19,858 & 20,019 & 19,809 & 19,893 & 19,929 \\
\hline Brain & 16,446 & 16,581 & 16,541 & 17,069 & 17,000 & 17,542 & 17,661 & 17,975 & 18,204 & 18,543 & 18,709 & 18,587 & 18,703 & 18,727 \\
\hline $\begin{array}{l}\text { Cranial nerves other nervous } \\
\text { system }\end{array}$ & 1,165 & 1,249 & 1,225 & 1,198 & 1,353 & 1,337 & 1,332 & 1,226 & 1,308 & 1,315 & 1,310 & 1,222 & 1,190 & 1,202 \\
\hline Endocrine system & 19,093 & 21,074 & 22,594 & 1 & 25,856 & 28,353 & 31,030 & 33,174 & 36,000 & 39,386 & 41,693 & 42,584 & & 45,696 \\
\hline Thyroid & 17,385 & 19,322 & 20,731 & 22,557 & 23,905 & 26,263 & 28,946 & 31,071 & 33,816 & 37,089 & 39,506 & 40,322 & 41,814 & 43,523 \\
\hline Other endocrine including thymus & 1,708 & 1,752 & 1,863 & 1,844 & 1,951 & 2,090 & 2,084 & 2,103 & 2,184 & 2,297 & 2,187 & 2,262 & 2,110 & 2,173 \\
\hline Lym & 56,381 & 57,054 & 47 & 59,578 & 60,948 & 62,813 & 63,613 & & 65,116 & & 67,262 & 67,800 & & 67,005 \\
\hline Hodgl & 7,272 & 7,461 & 7,346 & 7,678 & 7,617 & 7,726 & 7,981 & 7,905 & 7,972 & 8,273 & 8,012 & 8,069 & 7,884 & 7,704 \\
\hline Non-Hodgkin lymphoma & 49,109 & 49,593 & 50,801 & 51,900 & 53,331 & 55,087 & 55,632 & 55,857 & 57,144 & 58,077 & 59,250 & 59,731 & 59,289 & 59,301 \\
\hline Myeloma & 14,184 & 14,849 & 15,308 & 15,608 & 15,901 & 16,548 & 16,879 & 16,946 & 17,236 & 18,122 & 18,828 & 19,416 & 19,942 & 20,284 \\
\hline Leuk & 88 & 33 & 57 & 08 & 70 & 80 & 52 & 14 & 37,875 & 08 & 39,192 & 14 & 30 & 41,569 \\
\hline Acute lymphoc) & 3,670 & 3,823 & 3,939 & 3,916 & 3,970 & 4,131 & 4,066 & 4,269 & 4,380 & 4,536 & 4,491 & 4,600 & 4,701 & 4,536 \\
\hline Chronic lymphocytic leukemia & 10,499 & 11,455 & 12,049 & 12,149 & 12,669 & 13,133 & 13,511 & 13,683 & 13,352 & 13,548 & 13,605 & 13,759 & 13,897 & 13,938 \\
\hline Acute myeloid leukemia & 9,535 & 10,089 & 10,192 & 9,895 & 10,143 & 10,132 & 10,015 & 10,292 & 10,407 & 10,824 & 10,938 & 12,208 & 12,448 & 12,883 \\
\hline Chronic myeloid leukemia & 4,122 & 4,305 & 4,343 & 4,104 & 4,302 & 4,437 & 4,370 & 4,612 & 4,643 & 4,758 & 5,045 & 5,278 & 5,305 & 5,223 \\
\hline Other leukemias & 4,862 & 4,861 & 4,844 & 4,744 & 4,886 & 4,747 & 4,790 & 4,758 & 5,093 & 4,842 & 5,113 & 5,369 & 5,179 & 4,989 \\
\hline Mesothelioma & 2,825 & 2,889 & 2,891 & 2,935 & 2,925 & 2,964 & 3,074 & 2,978 & 2,974 & 3,012 & 3,048 & 3,070 & 3,079 & 3,002 \\
\hline Kaposi Sarcoma & 1,406 & 1,399 & 1,391 & 1,306 & 1,349 & 1,315 & 1,355 & 1,226 & 1,263 & 1,191 & 1,172 & 1,112 & 1,113 & 1,036 \\
\hline Miscellaneous & 32,014 & 31,788 & 31,511 & 31,304 & 30,362 & 30,030 & 30,045 & 29,848 & 29,849 & 29,874 & 29,845 & 30,598 & 30,160 & 30,457 \\
\hline
\end{tabular}

Abbreviation: NOS = not otherwise specified.

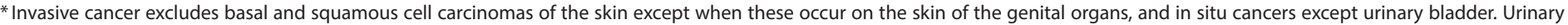
bladder cancer includes invasive and in situ.

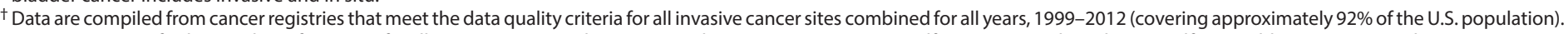

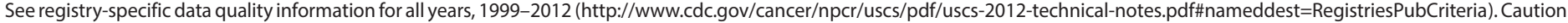
should be used when comparing number of cases and deaths because of potential differences in population coverage. 
TABLE 14. Reported number of cancer deaths, by primary cancer site and year — United States, 1999-2012*

\begin{tabular}{|c|c|c|c|c|c|c|c|c|c|c|c|c|c|c|}
\hline Cancer site & 1999 & 2000 & 2001 & 2002 & 2003 & 2004 & 2005 & 2006 & 2007 & 2008 & 2009 & 2010 & 2011 & 2012 \\
\hline All cancer sites combined & 549,829 & 553,080 & 553,760 & 557,264 & 556,890 & 553,880 & 559,303 & 559,880 & 562,867 & 565,460 & 567,614 & 574,738 & 576,685 & 582,607 \\
\hline Oral cavity and pharynx & 7,486 & 7,492 & 7,701 & 7,737 & 7,777 & 7,826 & 7,773 & 7,720 & 8,067 & 8,019 & 7,922 & 8,474 & 8,657 & 8,924 \\
\hline Lip & 52 & 67 & 77 & 74 & 72 & 74 & 60 & 51 & 66 & 68 & 57 & 69 & 83 & 71 \\
\hline Tongue & 1,738 & 1,767 & 1,818 & 1,887 & 1,875 & 1,881 & 1,948 & 1,906 & 2,034 & 1,983 & 1,971 & 2,125 & 2,089 & 2,224 \\
\hline Salivary gland & 656 & 663 & 685 & 722 & 696 & 697 & 701 & 699 & 742 & 718 & 795 & 827 & 820 & 869 \\
\hline Floor of mouth & 180 & 153 & 135 & 149 & 147 & 122 & 135 & 114 & 102 & 99 & 109 & 93 & 84 & 75 \\
\hline Gum and other mouth & 1,215 & 1,213 & 1,179 & 1,152 & 1,176 & 1,168 & 1,118 & 1,108 & 1,109 & 1,160 & 1,091 & 1,212 & 1,245 & 1,275 \\
\hline Nasopharynx & 638 & 650 & 621 & 628 & 598 & 637 & 615 & 633 & 677 & 645 & 662 & 701 & 630 & 666 \\
\hline Tonsil & 543 & 518 & 612 & 638 & 608 & 592 & 647 & 654 & 688 & 724 & 736 & 795 & 842 & 879 \\
\hline Oropharynx & 600 & 547 & 592 & 606 & 626 & 658 & 655 & 650 & 721 & 734 & 720 & 806 & 861 & 891 \\
\hline Hypopharynx & 385 & 359 & 348 & 323 & 318 & 368 & 281 & 301 & 324 & 294 & 279 & 324 & 322 & 356 \\
\hline Other oral cavity and pharynx & 1,479 & 1,555 & 1,634 & 1,558 & 1,661 & 1,629 & 1,613 & 1,604 & 1,604 & 1,594 & 1,502 & 1,522 & 1,681 & 1,618 \\
\hline Digestive system & 130,070 & 131,455 & 131,726 & 132,541 & 133,010 & 132,215 & 133,562 & 135,140 & 136,419 & 138,469 & 139,200 & 142,680 & 144,007 & 147,024 \\
\hline Esophagus & 11,917 & 12,232 & 12,529 & 12,700 & 12,860 & 13,023 & 13,499 & 13,685 & 13,592 & 13,714 & 13,908 & 14,490 & 14,446 & 14,649 \\
\hline Stomach & 12,711 & 12,645 & 12,319 & 12,198 & 12,110 & 11,859 & 11,514 & 11,345 & 11,388 & 11,352 & 11,184 & 11,390 & 11,035 & 11,191 \\
\hline Small intestine & 1,036 & 1,057 & 1,082 & 1,017 & 1,070 & 1,115 & 1,117 & 1,091 & 1,083 & 1,192 & 1,195 & 1,218 & 1,256 & 1,293 \\
\hline Colon and rectum & 57,222 & 57,434 & 56,808 & 56,603 & 55,783 & 53,580 & 53,005 & 53,196 & 53,219 & 52,857 & 51,848 & 52,045 & 51,783 & 51,516 \\
\hline Colon excluding rectum & 48,962 & 49,019 & 48,292 & 47,987 & 47,248 & 44,988 & 44,325 & 44,331 & 44,247 & 43,650 & 42,471 & 42,245 & 42,181 & 41,867 \\
\hline Rectum and rectosigmoid junction & 8,260 & 8,415 & 8,516 & 8,616 & 8,535 & 8,592 & 8,680 & 8,865 & 8,972 & 9,207 & 9,377 & 9,800 & 9,602 & 9,649 \\
\hline Anus, anal canal, and anorectum & 462 & 492 & 511 & 539 & 555 & 589 & 583 & 623 & 644 & 718 & 818 & 813 & 863 & 889 \\
\hline Liver and intrahepatic bile duct & 12,382 & 12,916 & 13,351 & 14,047 & 14,706 & 15,321 & 16,075 & 16,525 & 17,146 & 18,213 & 19,352 & 20,304 & 21,608 & 22,972 \\
\hline Gallbladder & 2,059 & 1,949 & 1,971 & 1,907 & 1,915 & 1,936 & 1,989 & 2,000 & 1,914 & 1,971 & 2,048 & 2,105 & 2,101 & 2,102 \\
\hline Other biliary & 1,531 & 1,717 & 1,630 & 1,501 & 1,491 & 1,461 & 1,464 & 1,427 & 1,436 & 1,377 & 1,384 & 1,519 & 1,510 & 1,519 \\
\hline Pancreas & 29,081 & 29,331 & 29,802 & 30,263 & 30,777 & 31,771 & 32,759 & 33,454 & 34,117 & 35,234 & 35,628 & 36,888 & 37,344 & 38,797 \\
\hline Retroperitoneum & 220 & 262 & 220 & 219 & 191 & 219 & 238 & 190 & 204 & 226 & 186 & 202 & 219 & 207 \\
\hline $\begin{array}{l}\text { Peritoneum, omentum, and } \\
\text { mesentery }\end{array}$ & 429 & 503 & 543 & 616 & 602 & 686 & 648 & 698 & 721 & 695 & 703 & 702 & 807 & 750 \\
\hline Other digestive organs & 1,020 & 917 & 960 & 931 & 950 & 655 & 671 & 906 & 955 & 920 & 946 & 1,004 & 1,035 & 1,139 \\
\hline Respiratory system & 156,708 & 160,051 & 160,602 & 162,148 & 162,589 & 162,400 & 163,751 & 163,134 & 163,065 & 163,141 & 162,492 & 162,730 & 161,376 & 161,851 \\
\hline Nose, nasal cavity, and middle ear & 456 & 419 & 485 & 444 & 457 & 458 & 484 & 426 & 475 & 516 & 530 & 495 & 416 & 458 \\
\hline Larynx & 3,815 & 3,861 & 3,797 & 3,722 & 3,791 & 3,668 & 3,796 & 3,821 & 3,634 & 3,760 & 3,630 & 3,691 & 3,732 & 3,662 \\
\hline Lung and bronchus & 152,061 & 155,426 & 155,969 & 157,630 & 157,990 & 158,006 & 159,217 & 158,599 & 158,683 & 158,592 & 158,081 & 158,248 & 156,953 & 157,423 \\
\hline Pleura & 99 & 76 & 84 & 73 & 76 & 78 & 67 & 64 & 48 & 54 & 55 & 54 & 61 & 82 \\
\hline $\begin{array}{l}\text { Trachea, mediastinum, and other } \\
\text { respiratory organs }\end{array}$ & 277 & 269 & 267 & 279 & 275 & 190 & 187 & 224 & 225 & 219 & 196 & 242 & 214 & 226 \\
\hline Bones and joints & 1,224 & 1,212 & 1,298 & 1,194 & 1,262 & 1,301 & 1,391 & 1,340 & 1,362 & 1,357 & 1,384 & 1,378 & 1,423 & 1,399 \\
\hline Soft tissue including heart & 3,679 & 3,693 & 3,646 & 3,554 & 3,651 & 3,722 & 3,849 & 3,960 & 4,023 & 4,093 & 4,229 & 4,376 & 4,408 & 4,559 \\
\hline Skin excluding basal and squ & 9,530 & 9,672 & 9,992 & 9,904 & 10,214 & 10,301 & 10,798 & 11,068 & 11,234 & 11,337 & 12,130 & 12,089 & 12,212 & 12,463 \\
\hline Melanoma of the skin & 7,215 & 7,420 & 7,542 & 7,513 & 7,818 & 7,952 & 8,345 & 8,441 & 8,461 & 8,623 & 9,199 & 9,154 & 9,128 & 9,251 \\
\hline Other nonepithelial skin & 2,315 & 2,252 & 2,450 & 2,391 & 2,396 & 2,349 & 2,453 & 2,627 & 2,773 & 2,714 & 2,931 & 2,935 & 3,084 & 3,212 \\
\hline breast & 41,528 & 42,300 & 41,809 & 41,883 & 41,998 & 41,316 & 41,491 & 41,209 & 40,969 & 41,026 & 41,076 & 41,435 & 41,374 & 41,555 \\
\hline Female breast & 41,144 & 41,872 & 41,394 & 41,514 & 41,619 & 40,954 & 41,116 & 40,820 & 40,598 & 40,589 & 40,676 & 40,996 & 40,931 & 41,150 \\
\hline Male breast & 384 & 428 & 415 & 369 & 379 & 362 & 375 & 389 & 371 & 437 & 400 & 439 & 443 & 405 \\
\hline
\end{tabular}

See table footnotes on page 54 . 
TABLE 14. (Continued) Reported number of cancer deaths, by primary cancer site and year - United States, 1999-2012*

\begin{tabular}{|c|c|c|c|c|c|c|c|c|c|c|c|c|c|c|}
\hline Cancer site & 1999 & 2000 & 2001 & 2002 & 2003 & 2004 & 2005 & 2006 & 2007 & 2008 & 2009 & 2010 & 2011 & 2012 \\
\hline Female genital system & 25,881 & 26,411 & 26,838 & 27,091 & 27,011 & 27,049 & 27,259 & 27,848 & 27,739 & 27,813 & 27,817 & 28,770 & 29,027 & 29,405 \\
\hline Cervix & 4,204 & 4,200 & 4,092 & 3,952 & 3,919 & 3,850 & 3,924 & 3,976 & 4,021 & 4,008 & 3,909 & 3,939 & 4,092 & 4,074 \\
\hline Corpus and uterus, NOS & 6,468 & 6,585 & 6,783 & 6,853 & 6,899 & 6,990 & 7,096 & 7,384 & 7,456 & 7,675 & 7,713 & 8,402 & 8,641 & 8,911 \\
\hline Corpus & 3,121 & 3,139 & 3,185 & 3,187 & 3,261 & 3,272 & 3,259 & 3,449 & 3,377 & 3,436 & 3,333 & 3,644 & 3,714 & 3,812 \\
\hline Uterus, NOS & 3,347 & 3,446 & 3,598 & 3,666 & 3,638 & 3,718 & 3,837 & 3,935 & 4,079 & 4,239 & 4,380 & 4,758 & 4,927 & 5,099 \\
\hline Ovary & 13,627 & 14,060 & 14,414 & 14,682 & 14,657 & 14,716 & 14,787 & 14,857 & 14,621 & 14,362 & 14,436 & 14,572 & 14,346 & 14,404 \\
\hline Vagina & 403 & 405 & 382 & 378 & 391 & 416 & 382 & 396 & 376 & 417 & 398 & 423 & 428 & 429 \\
\hline Vulva & 762 & 752 & 765 & 794 & 775 & 806 & 809 & 862 & 865 & 921 & 946 & 942 & 1,022 & 1,034 \\
\hline Other female genital organs & 417 & 409 & 402 & 432 & 370 & 271 & 261 & 373 & 400 & 430 & 415 & 492 & 498 & 553 \\
\hline Male genital system & 32,349 & 31,675 & 31,300 & 31,084 & 30,176 & 29,627 & 29,514 & 29,022 & 29,703 & 29,120 & 28,744 & 29,276 & 28,630 & 27,955 \\
\hline Prostate & 31,728 & 31,078 & 30,719 & 30,446 & 29,554 & 29,002 & 28,905 & 28,372 & 29,093 & 28,471 & 28,088 & 28,560 & 27,970 & 27,244 \\
\hline Testis & 378 & 338 & 335 & 393 & 344 & 357 & 359 & 358 & 326 & 358 & 376 & 399 & 380 & 386 \\
\hline Penis & 202 & 217 & 205 & 209 & 250 & 231 & 217 & 245 & 246 & 246 & 234 & 258 & 239 & 273 \\
\hline Other male genital organs & 41 & 42 & 41 & 36 & 28 & 37 & 33 & 47 & 38 & 45 & 46 & 59 & 41 & 52 \\
\hline Urinary system & 23,666 & 24,344 & 24,910 & 25,443 & 25,422 & 25,928 & 26,404 & 26,649 & 27,319 & 27,682 & 27,941 & 28,726 & 29,317 & 29,594 \\
\hline Urinary bladder & 11,910 & 12,002 & 12,225 & 12,627 & 12,483 & 13,030 & 13,253 & 13,474 & 13,843 & 14,036 & 14,201 & 14,730 & 15,014 & 15,245 \\
\hline Kidney and renal pelvis & 11,116 & 11,736 & 12,078 & 12,165 & 12,286 & 12,313 & 12,517 & 12,379 & 12,703 & 12,895 & 12,995 & 13,219 & 13,559 & 13,518 \\
\hline Ureter & 345 & 302 & 294 & 297 & 323 & 334 & 347 & 361 & 340 & 354 & 371 & 350 & 338 & 378 \\
\hline Other urinary organs & 295 & 304 & 313 & 354 & 330 & 251 & 287 & 435 & 433 & 397 & 374 & 427 & 406 & 453 \\
\hline Eye and orbit & 227 & 236 & 226 & 240 & 231 & 208 & 252 & 219 & 249 & 262 & 278 & 283 & 280 & 279 \\
\hline Brain and other nervous system & 12,765 & 12,655 & 12,609 & 12,830 & 12,901 & 12,829 & 13,152 & 12,886 & 13,234 & 13,724 & 14,176 & 14,164 & 14,491 & 15,276 \\
\hline Endocrine system & 2,146 & 2,210 & 2,299 & 2,231 & 2,155 & 2,272 & 2,354 & 2,404 & 2,488 & 2,555 & 2,634 & 2,641 & 2,689 & 2,660 \\
\hline Thyroid & 1,241 & 1,328 & 1,354 & 1,367 & 1,312 & 1,409 & 1,462 & 1,518 & 1,562 & 1,649 & 1,707 & 1,686 & 1,747 & 1,690 \\
\hline Other endocrine including thymus & 905 & 882 & 945 & 864 & 843 & 863 & 892 & 886 & 926 & 906 & 927 & 955 & 942 & 970 \\
\hline Lymphomas & 24,205 & 24,016 & 23,628 & 23,262 & 22,822 & 22,214 & 22,145 & 21,920 & 21,799 & 21,539 & 21,639 & 21,525 & 21,485 & 21,518 \\
\hline Hodgkin lymphoma & 1,403 & 1,287 & 1,323 & 1,352 & 1,347 & 1,276 & 1,272 & 1,327 & 1,271 & 1,171 & 1,250 & 1,231 & 1,168 & 1,130 \\
\hline Non-Hodgkin lymphoma & 22,802 & 22,729 & 22,305 & 21,910 & 21,475 & 20,938 & 20,873 & 20,593 & 20,528 & 20,368 & 20,389 & 20,294 & 20,317 & 20,388 \\
\hline Myeloma & 10,508 & 10,639 & 10,714 & 10,913 & 10,809 & 10,578 & 10,758 & 10,712 & 10,872 & 10,606 & 10,690 & 11,022 & 11,411 & 11,821 \\
\hline Leukemias & 21,071 & 21,397 & 21,532 & 21,581 & 21,608 & 21,472 & 21,716 & 22,016 & 21,928 & 22,431 & 22,688 & 22,673 & 23,194 & 23,309 \\
\hline Acute lymphocytic leukemia & 1,361 & 1,395 & 1,433 & 1,432 & 1,429 & 1,371 & 1,460 & 1,393 & 1,418 & 1,424 & 1,423 & 1,436 & 1,432 & 1,408 \\
\hline Chronic lymphocytic leukemia & 4,476 & 4,323 & 4,386 & 4,443 & 4,476 & 4,342 & 4,391 & 4,498 & 4,471 & 4,395 & 4,557 & 4,486 & 4,608 & 4,598 \\
\hline Acute myeloid leukemia & 6,932 & 7,413 & 7,749 & 7,914 & 8,126 & 8,214 & 8,267 & 8,539 & 8,568 & 8,962 & 9,223 & 9,150 & 9,491 & 9,484 \\
\hline Chronic myeloid leukemia & 1,788 & 1,802 & 1,649 & 1,367 & 1,233 & 1,164 & 1,067 & 1,077 & 984 & 1,000 & 1,003 & 1,019 & 1,091 & 1,017 \\
\hline Other leukemias & 6,514 & 6,464 & 6,315 & 6,425 & 6,344 & 6,381 & 6,531 & 6,509 & 6,487 & 6,650 & 6,482 & 6,582 & 6,572 & 6,802 \\
\hline Mesothelioma & 2,343 & 2,384 & 2,371 & 2,430 & 2,476 & 2,504 & 2,553 & 2,452 & 2,432 & 2,538 & 2,606 & 2,574 & 2,651 & 2,686 \\
\hline Miscellaneous & 44,401 & 41,176 & 40,519 & 41,144 & 40,723 & 40,070 & 40,534 & 40,140 & 39,920 & 39,700 & 39,926 & 39,886 & 40,002 & 40,276 \\
\hline
\end{tabular}

Abbreviation: NOS $=$ not otherwise specified.

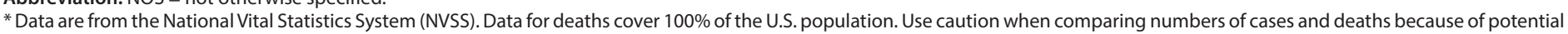
differences in population coverage. For more information, see USCS technical notes (http://www.cdc.gov/cancer/npcr/uscs/pdf/uscs-2012-technical-notes.pdf). 
TABLE 15. Age-adjusted rate* of invasive ${ }^{\dagger}$ cancer cases, by primary cancer site and year — United States, 1999-2012 ${ }^{\S}$

\begin{tabular}{|c|c|c|c|c|c|c|c|c|c|c|c|c|c|c|}
\hline Cancer site & 1999 & 2000 & 2001 & 2002 & 2003 & 2004 & 2005 & 2006 & 2007 & 2008 & 2009 & 2010 & 2011 & 2012 \\
\hline All cancer sites combined & 483.9 & 485.1 & 487.2 & 483.7 & 473.1 & 471.6 & 470.9 & 473.4 & 476.5 & 471.8 & 466.0 & 453.2 & 450.9 & 433.7 \\
\hline Oral cavity and pharynx & 10.9 & 10.8 & 10.8 & 10.8 & 10.8 & 10.8 & 10.7 & 10.8 & 11.1 & 11.3 & 11.2 & 11.2 & 11.4 & 11.2 \\
\hline Lip & 1.0 & 0.9 & 0.9 & 0.9 & 0.8 & 0.7 & 0.7 & 0.7 & 0.7 & 0.6 & 0.6 & 0.6 & 0.6 & 0.5 \\
\hline Tongue & 2.6 & 2.7 & 2.7 & 2.8 & 2.8 & 2.9 & 2.9 & 3.0 & 3.1 & 3.3 & 3.3 & 3.2 & 3.4 & 3.4 \\
\hline Salivary gland & 1.2 & 1.2 & 1.2 & 1.2 & 1.2 & 1.2 & 1.2 & 1.3 & 1.3 & 1.3 & 1.3 & 1.2 & 1.2 & 1.3 \\
\hline Floor of mouth & 0.8 & 0.8 & 0.8 & 0.8 & 0.7 & 0.7 & 0.6 & 0.7 & 0.6 & 0.6 & 0.6 & 0.6 & 0.6 & 0.5 \\
\hline Gum and other mouth & 1.7 & 1.6 & 1.6 & 1.6 & 1.6 & 1.6 & 1.5 & 1.5 & 1.6 & 1.6 & 1.5 & 1.6 & 1.6 & 1.5 \\
\hline Nasopharynx & 0.6 & 0.6 & 0.5 & 0.6 & 0.6 & 0.6 & 0.6 & 0.5 & 0.6 & 0.6 & 0.5 & 0.5 & 0.5 & 0.5 \\
\hline Tonsil & 1.4 & 1.4 & 1.4 & 1.5 & 1.6 & 1.6 & 1.6 & 1.7 & 1.7 & 1.8 & 2.0 & 1.9 & 2.0 & 2.0 \\
\hline Oropharynx & 0.4 & 0.4 & 0.4 & 0.4 & 0.5 & 0.5 & 0.5 & 0.5 & 0.5 & 0.5 & 0.5 & 0.5 & 0.5 & 0.5 \\
\hline Hypopharynx & 0.9 & 0.8 & 0.8 & 0.8 & 0.8 & 0.8 & 0.7 & 0.7 & 0.7 & 0.7 & 0.7 & 0.6 & 0.6 & 0.6 \\
\hline Other oral cavity and pharynx & 0.4 & 0.4 & 0.4 & 0.3 & 0.3 & 0.3 & 0.3 & 0.2 & 0.3 & 0.3 & 0.2 & 0.3 & 0.3 & 0.3 \\
\hline Digestive system & 91.8 & 91.6 & 91.2 & 90.1 & 89.7 & 88.7 & 87.3 & 86.3 & 85.6 & 84.8 & 82.9 & 80.7 & 80.1 & 78.4 \\
\hline Esophagus & 5.0 & 5.0 & 5.0 & 4.9 & 5.0 & 5.1 & 5.0 & 5.0 & 4.9 & 5.0 & 4.9 & 4.6 & 4.6 & 4.5 \\
\hline Stomach & 7.6 & 7.5 & 7.4 & 7.3 & 7.3 & 7.2 & 7.0 & 6.8 & 6.8 & 6.7 & 6.6 & 6.8 & 6.7 & 6.6 \\
\hline Small intestine & 1.7 & 1.7 & 1.8 & 1.8 & 1.9 & 2.0 & 2.0 & 2.1 & 2.1 & 2.2 & 2.2 & 2.3 & 2.3 & 2.3 \\
\hline Colon and rectum & 56.4 & 56.0 & 55.1 & 53.8 & 52.6 & 51.0 & 49.6 & 48.1 & 46.9 & 45.7 & 43.4 & 41.3 & 40.4 & 38.8 \\
\hline Colon excluding rectum & 41.1 & 40.9 & 40.4 & 39.4 & 38.5 & 37.3 & 36.0 & 35.0 & 34.1 & 33.2 & 31.3 & 29.7 & 29.0 & 27.7 \\
\hline Rectum and rectosigmoid junction & 15.2 & 15.1 & 14.8 & 14.4 & 14.1 & 13.8 & 13.6 & 13.1 & 12.8 & 12.5 & 12.1 & 11.6 & 11.4 & 11.1 \\
\hline Anus, anal canal, and anorectum & 1.4 & 1.4 & 1.4 & 1.4 & 1.5 & 1.6 & 1.6 & 1.6 & 1.7 & 1.7 & 1.8 & 1.7 & 1.8 & 1.8 \\
\hline Liver and Intrahepatic bile duct & 4.9 & 5.2 & 5.2 & 5.4 & 5.6 & 5.9 & 6.1 & 6.3 & 6.8 & 7.0 & 7.4 & 7.4 & 7.6 & 7.8 \\
\hline Gallbladder & 1.2 & 1.2 & 1.2 & 1.1 & 1.2 & 1.1 & 1.2 & 1.2 & 1.1 & 1.1 & 1.2 & 1.2 & 1.1 & 1.1 \\
\hline Other biliary & 1.3 & 1.4 & 1.6 & 1.7 & 1.7 & 1.7 & 1.7 & 1.7 & 1.7 & 1.7 & 1.8 & 1.8 & 1.8 & 1.7 \\
\hline Pancreas & 11.1 & 11.2 & 11.3 & 11.2 & 11.5 & 11.6 & 11.8 & 12.0 & 12.0 & 12.3 & 12.2 & 12.3 & 12.4 & 12.4 \\
\hline Retroperitoneum & 0.4 & 0.4 & 0.4 & 0.4 & 0.4 & 0.4 & 0.4 & 0.4 & 0.4 & 0.4 & 0.4 & 0.4 & 0.4 & 0.4 \\
\hline $\begin{array}{l}\text { Peritoneum, omentum, and } \\
\text { mesentery }\end{array}$ & 0.4 & 0.5 & 0.5 & 0.6 & 0.6 & 0.7 & 0.6 & 0.6 & 0.7 & 0.7 & 0.6 & 0.6 & 0.6 & 0.6 \\
\hline Other digestive organs & 0.3 & 0.3 & 0.4 & 0.4 & 0.4 & 0.4 & 0.4 & 0.4 & 0.4 & 0.4 & 0.4 & 0.4 & 0.5 & 0.5 \\
\hline Respiratory system & 76.3 & 75.6 & 75.4 & 74.9 & 74.7 & 73.9 & 74.0 & 73.1 & 72.1 & 71.3 & 69.9 & 67.5 & 65.8 & 64.0 \\
\hline Nose, nasal cavity, and middle ear & 0.7 & 0.7 & 0.7 & 0.7 & 0.7 & 0.7 & 0.7 & 0.7 & 0.7 & 0.7 & 0.7 & 0.7 & 0.7 & 0.7 \\
\hline Larynx & 4.6 & 4.5 & 4.4 & 4.1 & 4.1 & 4.1 & 4.0 & 3.9 & 3.9 & 3.8 & 3.7 & 3.6 & 3.5 & 3.3 \\
\hline Lung and bronchus & 70.7 & 70.1 & 70.1 & 69.9 & 69.7 & 68.8 & 69.1 & 68.2 & 67.3 & 66.6 & 65.3 & 62.9 & 61.5 & 59.8 \\
\hline Pleura & 0 & 0 & 0 & 0 & 0 & 0 & 0 & 0 & 0 & 0 & 0 & 0 & 0 & 0 \\
\hline $\begin{array}{l}\text { Trachea, mediastinum, and other } \\
\text { respiratory organs }\end{array}$ & 0.2 & 0.2 & 0.2 & 0.2 & 0.2 & 0.2 & 0.2 & 0.2 & 0.2 & 0.2 & 0.2 & 0.2 & 0.2 & 0.2 \\
\hline Bones and joints & 1.0 & 0.9 & 1.0 & 1.0 & 1.0 & 1.0 & 1.0 & 1.0 & 1.0 & 0.9 & 1.0 & 0.9 & 0.9 & 0.9 \\
\hline Soft tissue including heart & 2.9 & 3.0 & 3.0 & 3.0 & 3.1 & 3.2 & 3.3 & 3.2 & 3.3 & 3.3 & 3.3 & 3.3 & 3.2 & 3.2 \\
\hline Skin excluding basal and squamous & 16.6 & 17.7 & 18.7 & 19.1 & 18.9 & 19.8 & 21.0 & 20.7 & 21.1 & 21.6 & 21.9 & 21.5 & 21.9 & 21.7 \\
\hline Melanoma of the skin & 15.3 & 16.2 & 17.2 & 17.6 & 17.4 & 18.2 & 19.4 & 19.1 & 19.5 & 19.9 & 20.2 & 19.8 & 20.2 & 20.1 \\
\hline Other nonepithelial skin & 1.3 & 1.4 & 1.5 & 1.5 & 1.5 & 1.5 & 1.6 & 1.6 & 1.6 & 1.7 & 1.7 & 1.6 & 1.6 & 1.6 \\
\hline Male and female breast & 74.3 & 72.7 & 72.3 & 70.6 & 66.9 & 66.1 & 65.7 & 66.0 & 66.7 & 67.2 & 67.6 & 65.3 & 66.3 & 65.7 \\
\hline Female breast & 135.2 & 132.8 & 132.3 & 129.3 & 122.9 & 121.5 & 121.0 & 121.9 & 123.2 & 124.4 & 125.3 & 121.2 & 123.2 & 122.3 \\
\hline Male breast & 1.4 & 1.3 & 1.2 & 1.3 & 1.3 & 1.3 & 1.3 & 1.3 & 1.3 & 1.4 & 1.3 & 1.4 & 1.3 & 1.3 \\
\hline
\end{tabular}

See table footnotes on page 56. 
TABLE 15. (Continued) Age-adjusted rate* of invasive ${ }^{\dagger}$ cancer cases, by primary cancer site and year - United States, 1999-2012 $\$$

\begin{tabular}{|c|c|c|c|c|c|c|c|c|c|c|c|c|c|c|}
\hline Cancer site & 1999 & 2000 & 2001 & 2002 & 2003 & 2004 & 2005 & 2006 & 2007 & 2008 & 2009 & 2010 & 2011 & 2012 \\
\hline Female genital system & 51.9 & 51.6 & 51.4 & 50.4 & 49.0 & 48.9 & 49.1 & 49.1 & 49.3 & 49.6 & 49.9 & 49.0 & 49.1 & 48.9 \\
\hline Cervix & 9.7 & 9.6 & 9.1 & 8.7 & 8.4 & 8.2 & 8.2 & 8.1 & 8.1 & 7.9 & 8.0 & 7.6 & 7.5 & 7.4 \\
\hline Corpus and uterus, NOS & 24.2 & 23.8 & 24.3 & 24.0 & 23.2 & 23.8 & 24.0 & 24.2 & 24.5 & 24.9 & 25.4 & 25.4 & 25.7 & 25.8 \\
\hline Corpus & 23.4 & 23.1 & 23.6 & 23.3 & 22.5 & 23.0 & 23.3 & 23.4 & 23.8 & 24.1 & 24.6 & 24.6 & 24.9 & 25.0 \\
\hline Uterus, NOS & 0.8 & 0.8 & 0.8 & 0.8 & 0.8 & 0.7 & 0.7 & 0.7 & 0.7 & 0.8 & 0.8 & 0.8 & 0.8 & 0.8 \\
\hline Ovary & 14.3 & 14.4 & 14.2 & 13.8 & 13.6 & 13.2 & 13.1 & 13.0 & 12.7 & 12.7 & 12.2 & 11.8 & 11.6 & 11.3 \\
\hline Vagina & 0.7 & 0.8 & 0.7 & 0.7 & 0.7 & 0.7 & 0.6 & 0.7 & 0.7 & 0.7 & 0.7 & 0.7 & 0.7 & 0.7 \\
\hline Vulva & 2.4 & 2.3 & 2.4 & 2.4 & 2.4 & 2.3 & 2.4 & 2.4 & 2.5 & 2.5 & 2.5 & 2.5 & 2.5 & 2.6 \\
\hline Other female genital organs & 0.6 & 0.7 & 0.7 & 0.7 & 0.7 & 0.7 & 0.7 & 0.7 & 0.8 & 0.9 & 0.9 & 1.0 & 1.1 & 1.2 \\
\hline Male genital system & 176.5 & 178.4 & 180.6 & 178.4 & 163.7 & 159.7 & 156.4 & 166.0 & 170.1 & 157.0 & 148.7 & 139.2 & 137.5 & 111.7 \\
\hline Prostate & 170.0 & 171.9 & 174.1 & 172.0 & 157.1 & 153.1 & 149.7 & 159.5 & 163.4 & 150.1 & 142.0 & 132.5 & 130.9 & 105.1 \\
\hline Testis & 5.3 & 5.4 & 5.4 & 5.3 & 5.5 & 5.5 & 5.6 & 5.5 & 5.5 & 5.7 & 5.6 & 5.6 & 5.5 & 5.5 \\
\hline Penis & 0.9 & 0.9 & 0.9 & 0.9 & 0.9 & 0.8 & 0.8 & 0.8 & 0.9 & 0.9 & 0.8 & 0.8 & 0.9 & 0.8 \\
\hline Other male genital organs & 0.2 & 0.3 & 0.2 & 0.2 & 0.2 & 0.2 & 0.2 & 0.2 & 0.2 & 0.3 & 0.2 & 0.2 & 0.2 & 0.2 \\
\hline Urinary system & 35.2 & 35.7 & 36.1 & 36.6 & 37.1 & 37.8 & 38.2 & 38.1 & 38.5 & 38.7 & 38.4 & 37.5 & 37.4 & 37.1 \\
\hline Urinary bladder & 22.0 & 22.1 & 21.9 & 22.0 & 22.0 & 22.2 & 22.2 & 21.5 & 21.7 & 21.5 & 21.3 & 20.9 & 20.7 & 20.4 \\
\hline Kidney and renal pelvis & 12.3 & 12.7 & 13.3 & 13.7 & 14.3 & 14.7 & 15.2 & 15.7 & 16.0 & 16.2 & 16.2 & 15.8 & 15.8 & 15.9 \\
\hline Ureter & 0.6 & 0.6 & 0.6 & 0.6 & 0.6 & 0.6 & 0.6 & 0.6 & 0.6 & 0.6 & 0.6 & 0.6 & 0.6 & 0.6 \\
\hline Other urinary organs & 0.3 & 0.3 & 0.3 & 0.2 & 0.3 & 0.3 & 0.3 & 0.3 & 0.3 & 0.3 & 0.3 & 0.3 & 0.3 & 0.3 \\
\hline Eye and orbit & 0.9 & 0.9 & 0.9 & 0.8 & 0.9 & 0.9 & 0.9 & 0.9 & 0.9 & 0.9 & 0.9 & 0.8 & 0.8 & 0.8 \\
\hline Brain and other nervous system & 6.9 & 6.9 & 6.8 & 6.9 & 6.8 & 6.9 & 6.9 & 6.8 & 6.8 & 6.8 & 6.8 & 6.6 & 6.5 & 6.4 \\
\hline Brain & 6.4 & 6.4 & 6.3 & 6.4 & 6.3 & 6.4 & 6.4 & 6.4 & 6.4 & 6.4 & 6.3 & 6.2 & 6.1 & 6.0 \\
\hline Cranial nerves other nervous system & 0.5 & 0.5 & 0.5 & 0.5 & 0.5 & 0.5 & 0.5 & 0.4 & 0.5 & 0.5 & 0.5 & 0.4 & 0.4 & 0.4 \\
\hline Endocrine system & 7.5 & 8.1 & 8.6 & 9.2 & 9.6 & 10.4 & 11.2 & 11.8 & 12.7 & 13.7 & 14.3 & 14.5 & 14.7 & 15.2 \\
\hline Thyroid & 6.8 & 7.4 & 7.9 & 8.5 & 8.9 & 9.6 & 10.5 & 11.1 & 11.9 & 12.9 & 13.6 & 13.7 & 14.0 & 14.5 \\
\hline Other endocrine including thymus & 0.7 & 0.7 & 0.7 & 0.7 & 0.7 & 0.8 & 0.8 & 0.8 & 0.8 & 0.8 & 0.7 & 0.8 & 0.7 & 0.7 \\
\hline Lymphomas & 22.2 & 22.1 & 22.2 & 22.4 & 22.6 & 22.9 & 22.9 & 22.6 & 22.6 & 22.7 & 22.6 & 22.4 & 21.8 & 21.3 \\
\hline Hodgkin lymphoma & 2.8 & 2.9 & 2.8 & 2.9 & 2.8 & 2.9 & 2.9 & 2.9 & 2.9 & 2.9 & 2.8 & 2.8 & 2.7 & 2.6 \\
\hline Non-Hodgkin lymphoma & 19.3 & 19.2 & 19.4 & 19.5 & 19.8 & 20.1 & 19.9 & 19.7 & 19.8 & 19.7 & 19.8 & 19.5 & 19.0 & 18.6 \\
\hline Myeloma & 5.6 & 5.8 & 5.9 & 5.9 & 5.9 & 6.0 & 6.0 & 5.9 & 5.9 & 6.1 & 6.2 & 6.3 & 6.3 & 6.3 \\
\hline Leukemias & 12.9 & 13.4 & 13.5 & 13.1 & 13.4 & 13.4 & 13.3 & 13.4 & 13.2 & 13.2 & 13.2 & 13.6 & 13.5 & 13.3 \\
\hline Acute lymphocytic leukemia & 1.4 & 1.5 & 1.5 & 1.5 & 1.5 & 1.6 & 1.5 & 1.6 & 1.6 & 1.6 & 1.6 & 1.6 & 1.7 & 1.6 \\
\hline Chronic lymphocytic leukemia & 4.1 & 4.5 & 4.6 & 4.6 & 4.7 & 4.8 & 4.8 & 4.8 & 4.6 & 4.6 & 4.5 & 4.4 & 4.4 & 4.3 \\
\hline Acute myeloid leukemia & 3.7 & 3.9 & 3.9 & 3.7 & 3.8 & 3.7 & 3.6 & 3.7 & 3.6 & 3.7 & 3.7 & 4.1 & 4.1 & 4.1 \\
\hline Chronic myeloid leukemia & 1.6 & 1.7 & 1.7 & 1.5 & 1.6 & 1.6 & 1.6 & 1.6 & 1.6 & 1.6 & 1.7 & 1.8 & 1.7 & 1.7 \\
\hline Other leukemias & 1.9 & 1.9 & 1.9 & 1.8 & 1.8 & 1.7 & 1.7 & 1.7 & 1.8 & 1.7 & 1.7 & 1.8 & 1.7 & 1.6 \\
\hline Mesothelioma & 1.1 & 1.1 & 1.1 & 1.1 & 1.1 & 1.1 & 1.1 & 1.1 & 1.0 & 1.0 & 1.0 & 1.0 & 1.0 & 1.0 \\
\hline Kaposi Sarcoma & 0.5 & 0.5 & 0.5 & 0.5 & 0.5 & 0.5 & 0.5 & 0.5 & 0.5 & 0.4 & 0.4 & 0.4 & 0.4 & 0.4 \\
\hline Miscellaneous & 12.6 & 12.4 & 12.1 & 11.8 & 11.2 & 10.9 & 10.7 & 10.5 & 10.3 & 10.1 & 9.8 & 9.9 & 9.6 & 9.4 \\
\hline
\end{tabular}

Abbreviation: NOS = not otherwise specified.

* Rates are the number of cases per 100,000 persons and are age-adjusted to the 2000 U.S. standard population (19 age groups - Census P25-1130). For more information, see USCS technical notes (http://www.cdc.gov/cancer/npcr/uscs/pdf/uscs-2012-technical-notes.pdf).

†Invasive cancer excludes basal and squamous cell carcinomas of the skin except when these occur on the skin of the genital organs, and in situ cancers except urinary bladder. Urinary bladder cancer includes invasive and in situ.

$\S$ Data are compiled from cancer registries that meet the data quality criteria for all invasive cancer sites combined for all years, 1999-2012 (covering approximately 92\% of the U.S. population). See registry-specific data quality information for all years, 1999-2012 (http://www.cdc.gov/cancer/npcr/uscs/pdf/uscs-2012-technical-notes.pdf\#nameddest=RegistriesPubCriteria). Caution should be used when comparing incidence and death rates because of potential differences in population coverage. 
TABLE 16. Age-adjusted rate* of cancer deaths, by primary cancer site and year — United States, 1999-2012 ${ }^{\dagger}$

\begin{tabular}{|c|c|c|c|c|c|c|c|c|c|c|c|c|c|c|}
\hline Cancer site & 1999 & 2000 & 2001 & 2002 & 2003 & 2004 & 2005 & 2006 & 2007 & 2008 & 2009 & 2010 & 2011 & 2012 \\
\hline All cancer sites combined & 200.7 & 198.8 & 196.3 & 194.4 & 190.9 & 186.8 & 185.2 & 182.0 & 179.3 & 176.3 & 173.4 & 171.8 & 168.7 & 166.4 \\
\hline Oral cavity and pharynx & 2.7 & 2.7 & 2.7 & 2.7 & 2.6 & 2.6 & 2.5 & 2.5 & 2.5 & 2.5 & 2.4 & 2.5 & 2.5 & 2.5 \\
\hline Lip & 0 & 0 & 0 & 0 & 0 & 0 & 0 & 0 & 0 & 0 & 0 & 0 & 0 & 0 \\
\hline Tongue & 0.6 & 0.6 & 0.6 & 0.7 & 0.6 & 0.6 & 0.6 & 0.6 & 0.6 & 0.6 & 0.6 & 0.6 & 0.6 & 0.6 \\
\hline Salivary gland & 0.2 & 0.2 & 0.2 & 0.3 & 0.2 & 0.2 & 0.2 & 0.2 & 0.2 & 0.2 & 0.2 & 0.2 & 0.2 & 0.2 \\
\hline Floor of mouth & 0.1 & 0.1 & 0 & 0.1 & 0 & 0 & 0 & 0 & 0 & 0 & 0 & 0 & 0 & 0 \\
\hline Gum and other mouth & 0.4 & 0.4 & 0.4 & 0.4 & 0.4 & 0.4 & 0.4 & 0.4 & 0.4 & 0.4 & 0.3 & 0.4 & 0.4 & 0.4 \\
\hline Nasopharynx & 0.2 & 0.2 & 0.2 & 0.2 & 0.2 & 0.2 & 0.2 & 0.2 & 0.2 & 0.2 & 0.2 & 0.2 & 0.2 & 0.2 \\
\hline Tonsil & 0.2 & 0.2 & 0.2 & 0.2 & 0.2 & 0.2 & 0.2 & 0.2 & 0.2 & 0.2 & 0.2 & 0.2 & 0.2 & 0.2 \\
\hline Oropharynx & 0.2 & 0.2 & 0.2 & 0.2 & 0.2 & 0.2 & 0.2 & 0.2 & 0.2 & 0.2 & 0.2 & 0.2 & 0.2 & 0.2 \\
\hline Hypopharynx & 0.1 & 0.1 & 0.1 & 0.1 & 0.1 & 0.1 & 0.1 & 0.1 & 0.1 & 0.1 & 0.1 & 0.1 & 0.1 & 0.1 \\
\hline Other oral cavity and pharynx & 0.5 & 0.6 & 0.6 & 0.5 & 0.6 & 0.5 & 0.5 & 0.5 & 0.5 & 0.5 & 0.4 & 0.4 & 0.5 & 0.4 \\
\hline Digestive system & 47.5 & 47.3 & 46.7 & 46.2 & 45.6 & 44.5 & 44.1 & 43.8 & 43.3 & 43.0 & 42.3 & 42.3 & 41.8 & 41.6 \\
\hline Esophagus & 4.3 & 4.4 & 4.4 & 4.4 & 4.4 & 4.4 & 4.4 & 4.4 & 4.3 & 4.2 & 4.2 & 4.3 & 4.2 & 4.1 \\
\hline Stomach & 4.6 & 4.5 & 4.4 & 4.3 & 4.2 & 4.0 & 3.8 & 3.7 & 3.6 & 3.5 & 3.4 & 3.4 & 3.3 & 3.2 \\
\hline Small intestine & 0.4 & 0.4 & 0.4 & 0.4 & 0.4 & 0.4 & 0.4 & 0.4 & 0.3 & 0.4 & 0.4 & 0.4 & 0.4 & 0.4 \\
\hline Colon and rectum & 20.9 & 20.7 & 20.2 & 19.8 & 19.1 & 18.1 & 17.6 & 17.3 & 16.9 & 16.5 & 15.8 & 15.5 & 15.1 & 14.7 \\
\hline Colon excluding rectum & 17.9 & 17.6 & 17.1 & 16.8 & 16.2 & 15.2 & 14.7 & 14.4 & 14.1 & 13.6 & 13.0 & 12.6 & 12.3 & 12.0 \\
\hline Rectum and rectosigmoid junction & 3.0 & 3.0 & 3.0 & 3.0 & 2.9 & 2.9 & 2.9 & 2.9 & 2.8 & 2.9 & 2.8 & 2.9 & 2.8 & 2.7 \\
\hline Anus, anal canal, and anorectum & 0.2 & 0.2 & 0.2 & 0.2 & 0.2 & 0.2 & 0.2 & 0.2 & 0.2 & 0.2 & 0.2 & 0.2 & 0.2 & 0.3 \\
\hline Liver and intrahepatic bile duct & 4.5 & 4.6 & 4.7 & 4.9 & 5.0 & 5.1 & 5.3 & 5.3 & 5.4 & 5.6 & 5.8 & 5.9 & 6.1 & 6.3 \\
\hline Gallbladder & 0.8 & 0.7 & 0.7 & 0.7 & 0.7 & 0.7 & 0.7 & 0.7 & 0.6 & 0.6 & 0.6 & 0.6 & 0.6 & 0.6 \\
\hline Other biliary & 0.6 & 0.6 & 0.6 & 0.5 & 0.5 & 0.5 & 0.5 & 0.5 & 0.5 & 0.4 & 0.4 & 0.5 & 0.4 & 0.4 \\
\hline Pancreas & 10.6 & 10.5 & 10.6 & 10.6 & 10.5 & 10.7 & 10.8 & 10.9 & 10.8 & 11.0 & 10.8 & 11.0 & 10.9 & 11.0 \\
\hline Retroperitoneum & 0.1 & 0.1 & 0.1 & 0.1 & 0.1 & 0.1 & 0.1 & 0.1 & 0.1 & 0.1 & 0.1 & 0.1 & 0.1 & 0.1 \\
\hline $\begin{array}{l}\text { Peritoneum, omentum, and } \\
\text { mesentery }\end{array}$ & 0.2 & 0.2 & 0.2 & 0.2 & 0.2 & 0.2 & 0.2 & 0.2 & 0.2 & 0.2 & 0.2 & 0.2 & 0.2 & 0.2 \\
\hline Other digestive organs & 0.4 & 0.3 & 0.3 & 0.3 & 0.3 & 0.2 & 0.2 & 0.3 & 0.3 & 0.3 & 0.3 & 0.3 & 0.3 & 0.3 \\
\hline Respiratory system & 57.1 & 57.5 & 57.0 & 56.6 & 55.8 & 54.8 & 54.3 & 53.2 & 52.1 & 51.0 & 49.7 & 48.7 & 47.3 & 46.2 \\
\hline Nose, nasal cavity, and middle ear & 0.2 & 0.2 & 0.2 & 0.2 & 0.2 & 0.2 & 0.2 & 0.1 & 0.2 & 0.2 & 0.2 & 0.1 & 0.1 & 0.1 \\
\hline Larynx & 1.4 & 1.4 & 1.3 & 1.3 & 1.3 & 1.2 & 1.2 & 1.2 & 1.1 & 1.2 & 1.1 & 1.1 & 1.1 & 1.0 \\
\hline Lung and bronchus & 55.4 & 55.8 & 55.3 & 55.0 & 54.2 & 53.4 & 52.9 & 51.7 & 50.7 & 49.6 & 48.4 & 47.4 & 46.0 & 45.0 \\
\hline Pleura & 0 & 0 & 0 & 0 & 0 & 0 & 0 & 0 & 0 & 0 & 0 & 0 & 0 & 0 \\
\hline $\begin{array}{l}\text { Trachea, mediastinum, and other } \\
\text { respiratory organs }\end{array}$ & 0.1 & 0.1 & 0.1 & 0.1 & 0.1 & 0.1 & 0.1 & 0.1 & 0.1 & 0.1 & 0.1 & 0.1 & 0.1 & 0.1 \\
\hline Bones and joints & 0.4 & 0.4 & 0.5 & 0.4 & 0.4 & 0.4 & 0.5 & 0.4 & 0.4 & 0.4 & 0.4 & 0.4 & 0.4 & 0.4 \\
\hline Soft tissue including heart & 1.3 & 1.3 & 1.3 & 1.2 & 1.2 & 1.3 & 1.3 & 1.3 & 1.3 & 1.3 & 1.3 & 1.3 & 1.3 & 1.3 \\
\hline $\begin{array}{l}\text { Skin excluding basal and } \\
\text { squamous }\end{array}$ & 3.5 & 3.5 & 3.5 & 3.4 & 3.5 & 3.5 & 3.6 & 3.6 & 3.6 & 3.5 & 3.7 & 3.6 & 3.6 & 3.6 \\
\hline Melanoma of the skin & 2.6 & 2.7 & 2.7 & 2.6 & 2.7 & 2.7 & 2.8 & 2.7 & 2.7 & 2.7 & 2.8 & 2.7 & 2.7 & 2.7 \\
\hline Other nonepithelial skin & 0.8 & 0.8 & 0.9 & 0.8 & 0.8 & 0.8 & 0.8 & 0.8 & 0.9 & 0.8 & 0.9 & 0.9 & 0.9 & 0.9 \\
\hline Male and female breast & 15.2 & 15.2 & 14.8 & 14.5 & 14.3 & 13.8 & 13.6 & 13.2 & 12.9 & 12.7 & 12.4 & 12.3 & 12.0 & 11.8 \\
\hline Female breast & 26.6 & 26.6 & 26.0 & 25.6 & 25.3 & 24.5 & 24.1 & 23.6 & 23.0 & 22.6 & 22.2 & 21.9 & 21.5 & 21.3 \\
\hline Male breast & 0.3 & 0.4 & 0.4 & 0.3 & 0.3 & 0.3 & 0.3 & 0.3 & 0.3 & 0.3 & 0.3 & 0.3 & 0.3 & 0.3 \\
\hline
\end{tabular}

See table footnotes on page 58 . 
TABLE 16. (Continued) Age-adjusted rate* of cancer deaths, by primary cancer site and year - United States, 1999-2012 ${ }^{\dagger}$

\begin{tabular}{|c|c|c|c|c|c|c|c|c|c|c|c|c|c|c|}
\hline Cancer site & 1999 & 2000 & 2001 & 2002 & 2003 & 2004 & 2005 & 2006 & 2007 & 2008 & 2009 & 2010 & 2011 & 2012 \\
\hline Female genital system & 16.7 & 16.7 & 16.8 & 16.7 & 16.4 & 16.2 & 16.0 & 16.1 & 15.8 & 15.5 & 15.2 & 15.5 & 15.3 & 15.2 \\
\hline Cervix & 2.8 & 2.8 & 2.7 & 2.6 & 2.5 & 2.4 & 2.4 & 2.4 & 2.4 & 2.4 & 2.3 & 2.3 & 2.3 & 2.3 \\
\hline Corpus and uterus, NOS & 4.1 & 4.1 & 4.2 & 4.2 & 4.1 & 4.1 & 4.1 & 4.2 & 4.2 & 4.2 & 4.2 & 4.5 & 4.5 & 4.5 \\
\hline Corpus & 2.0 & 2.0 & 2.0 & 1.9 & 2.0 & 1.9 & 1.9 & 2.0 & 1.9 & 1.9 & 1.8 & 1.9 & 1.9 & 1.9 \\
\hline Uterus, NOS & 2.1 & 2.1 & 2.2 & 2.2 & 2.2 & 2.2 & 2.2 & 2.2 & 2.3 & 2.3 & 2.4 & 2.5 & 2.6 & 2.6 \\
\hline Ovary & 8.8 & 8.9 & 9.0 & 9.0 & 8.9 & 8.8 & 8.7 & 8.6 & 8.3 & 8.0 & 7.9 & 7.8 & 7.5 & 7.4 \\
\hline Vagina & 0.2 & 0.2 & 0.2 & 0.2 & 0.2 & 0.2 & 0.2 & 0.2 & 0.2 & 0.2 & 0.2 & 0.2 & 0.2 & 0.2 \\
\hline Vulva & 0.5 & 0.4 & 0.5 & 0.5 & 0.4 & 0.5 & 0.5 & 0.5 & 0.5 & 0.5 & 0.5 & 0.5 & 0.5 & 0.5 \\
\hline Other female genital organs & 0.3 & 0.3 & 0.3 & 0.3 & 0.2 & 0.2 & 0.2 & 0.2 & 0.2 & 0.2 & 0.2 & 0.3 & 0.3 & 0.3 \\
\hline Male genital system & 32.1 & 30.9 & 30.0 & 29.2 & 27.7 & 26.7 & 25.8 & 24.7 & 24.7 & 23.5 & 22.6 & 22.3 & 21.2 & 20.0 \\
\hline Prostate & 31.6 & 30.4 & 29.5 & 28.7 & 27.2 & 26.2 & 25.4 & 24.2 & 24.2 & 23.0 & 22.1 & 21.8 & 20.8 & 19.6 \\
\hline Testis & 0.3 & 0.2 & 0.2 & 0.3 & 0.2 & 0.3 & 0.2 & 0.2 & 0.2 & 0.2 & 0.3 & 0.3 & 0.3 & 0.3 \\
\hline Penis & 0.2 & 0.2 & 0.2 & 0.2 & 0.2 & 0.2 & 0.2 & 0.2 & 0.2 & 0.2 & 0.2 & 0.2 & 0.2 & 0.2 \\
\hline Other male genital organs & 0 & 0 & 0 & 0 & 0 & 0 & 0 & 0 & 0 & 0 & 0 & 0 & 0 & 0 \\
\hline Urinary system & 8.7 & 8.8 & 8.8 & 8.9 & 8.7 & 8.8 & 8.8 & 8.7 & 8.7 & 8.6 & 8.5 & 8.6 & 8.6 & 8.5 \\
\hline Urinary bladder & 4.4 & 4.3 & 4.3 & 4.4 & 4.3 & 4.4 & 4.4 & 4.4 & 4.4 & 4.4 & 4.4 & 4.4 & 4.4 & 4.4 \\
\hline Kidney and renal pelvis & 4.1 & 4.2 & 4.3 & 4.2 & 4.2 & 4.1 & 4.1 & 4.0 & 4.0 & 4.0 & 3.9 & 3.9 & 3.9 & 3.8 \\
\hline Ureter & 0.1 & 0.1 & 0.1 & 0.1 & 0.1 & 0.1 & 0.1 & 0.1 & 0.1 & 0.1 & 0.1 & 0.1 & 0.1 & 0.1 \\
\hline Other urinary organs & 0.1 & 0.1 & 0.1 & 0.1 & 0.1 & 0.1 & 0.1 & 0.1 & 0.1 & 0.1 & 0.1 & 0.1 & 0.1 & 0.1 \\
\hline Eye and orbit & 0.1 & 0.1 & 0.1 & 0.1 & 0.1 & 0.1 & 0.1 & 0.1 & 0.1 & 0.1 & 0.1 & 0.1 & 0.1 & 0.1 \\
\hline Brain and other nervous system & 4.6 & 4.5 & 4.4 & 4.5 & 4.4 & 4.3 & 4.3 & 4.2 & 4.2 & 4.3 & 4.3 & 4.2 & 4.3 & 4.4 \\
\hline Endocrine system & 0.8 & 0.8 & 0.8 & 0.8 & 0.7 & 0.8 & 0.8 & 0.8 & 0.8 & 0.8 & 0.8 & 0.8 & 0.8 & 0.8 \\
\hline Thyroid & 0.5 & 0.5 & 0.5 & 0.5 & 0.4 & 0.5 & 0.5 & 0.5 & 0.5 & 0.5 & 0.5 & 0.5 & 0.5 & 0.5 \\
\hline Other endocrine including thymus & 0.3 & 0.3 & 0.3 & 0.3 & 0.3 & 0.3 & 0.3 & 0.3 & 0.3 & 0.3 & 0.3 & 0.3 & 0.3 & 0.3 \\
\hline Lymphomas & 8.8 & 8.6 & 8.4 & 8.1 & 7.8 & 7.5 & 7.4 & 7.2 & 7.0 & 6.8 & 6.7 & 6.5 & 6.4 & 6.2 \\
\hline Hodgkin lymphoma & 0.5 & 0.5 & 0.5 & 0.5 & 0.5 & 0.4 & 0.4 & 0.4 & 0.4 & 0.4 & 0.4 & 0.4 & 0.4 & 0.3 \\
\hline Non-Hodgkin lymphoma & 8.3 & 8.2 & 7.9 & 7.7 & 7.4 & 7.1 & 6.9 & 6.7 & 6.6 & 6.4 & 6.3 & 6.1 & 6.0 & 5.9 \\
\hline Myeloma & 3.8 & 3.8 & 3.8 & 3.8 & 3.7 & 3.6 & 3.6 & 3.5 & 3.5 & 3.3 & 3.3 & 3.3 & 3.4 & 3.4 \\
\hline Leukemias & 7.7 & 7.7 & 7.6 & 7.5 & 7.4 & 7.3 & 7.3 & 7.2 & 7.1 & 7.1 & 7.1 & 6.9 & 6.9 & 6.8 \\
\hline Acute lymphocytic leukemia & 0.5 & 0.5 & 0.5 & 0.5 & 0.5 & 0.5 & 0.5 & 0.5 & 0.5 & 0.5 & 0.5 & 0.5 & 0.4 & 0.4 \\
\hline Chronic lymphocytic leukemia & 1.6 & 1.6 & 1.6 & 1.6 & 1.5 & 1.5 & 1.5 & 1.5 & 1.4 & 1.4 & 1.4 & 1.4 & 1.4 & 1.3 \\
\hline Acute myeloid leukemia & 2.5 & 2.7 & 2.7 & 2.8 & 2.8 & 2.8 & 2.8 & 2.8 & 2.8 & 2.8 & 2.9 & 2.8 & 2.8 & 2.8 \\
\hline Chronic myeloid leukemia & 0.7 & 0.6 & 0.6 & 0.5 & 0.4 & 0.4 & 0.4 & 0.4 & 0.3 & 0.3 & 0.3 & 0.3 & 0.3 & 0.3 \\
\hline Other leukemias & 2.4 & 2.3 & 2.2 & 2.2 & 2.2 & 2.2 & 2.2 & 2.1 & 2.1 & 2.1 & 2.0 & 2.0 & 2.0 & 2.0 \\
\hline Mesothelioma & 0.9 & 0.9 & 0.8 & 0.9 & 0.9 & 0.9 & 0.9 & 0.8 & 0.8 & 0.8 & 0.8 & 0.8 & 0.8 & 0.8 \\
\hline Miscellaneous & 16.2 & 14.8 & 14.4 & 14.4 & 14.0 & 13.5 & 13.4 & 13.0 & 12.7 & 12.4 & 12.2 & 11.9 & 11.7 & 11.5 \\
\hline
\end{tabular}

Abbreviation: $\mathrm{NOS}=$ not otherwise specified

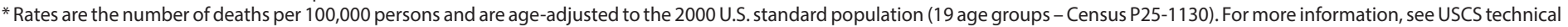
notes (http://www.cdc.gov/cancer/npcr/uscs/pdf/uscs-2012-technical-notes.pdf).

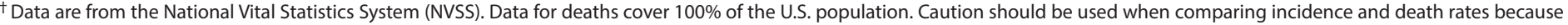
of potential differences in population coverage. 